quatrième série-tome $44 \quad$ fascicule 2 mars-avril 2011

$$
\begin{aligned}
& \text { ANNALES } \\
& \text { SCIENTIFIQUES } \\
& \text { de } \\
& \text { L'ECOLE } \\
& \text { NORMALE } \\
& \text { SUPERIEURE }
\end{aligned}
$$

Christopher DAVIS \& Andreas LANGER \& Thomas ZINK Overconvergent de Rham-Witt Cobomology 
Ann. Scient. Éc. Norm. Sup.

$4^{\text {e }}$ série, t. 44, 2011, p. 197 à 262

\title{
OVERCONVERGENT DE RHAM-WITT COHOMOLOGY
}

\author{
BY Christopher DAVIS, Andreas LANGER and Thomas ZINK
}

Abstract. - The goal of this work is to construct, for a smooth variety $X$ over a perfect field k of finite characteristic $p>0$, an overconvergent de Rham-Witt complex $W^{\dagger} \Omega_{X / k}$ as a suitable subcomplex of the de Rham-Witt complex of Deligne-Illusie. This complex, which is functorial in $X$, is a complex of étale sheaves and a differential graded algebra over the ring $W^{\dagger}\left(\theta_{X}\right)$ of overconvergent Witt-vectors. If $X$ is affine one proves that there is an isomorphism between Monsky-Washnitzer cohomology and (rational) overconvergent de Rham-Witt cohomology. Finally we define for a quasiprojective $X$ an isomorphism between the rational overconvergent de Rham-Witt cohomology and the rigid cohomology.

RÉSUMÉ. - Le but de ce travail est de construire, pour $X$ une variété lisse sur un corps parfait $k$ de caractéristique finie, un complexe de de Rham-Witt surconvergent $W^{\dagger} \Omega_{X / k}$ comme un sous-complexe convenable du complexe de de Rham-Witt de Deligne-Illusie. Ce complexe qui est fonctoriel en $X$ est un complexe de faisceaux étales et une algèbre différentielle graduée sur l'anneau $W^{\dagger}\left(\theta_{X}\right)$ des vecteurs de Witt surconvergents. Lorsque $X$ est affine, on démontre qu'il existe un isomorphisme canonique entre la cohomologie de Monsky-Washnitzer et la cohomologie (rationnelle) de de Rham-Witt surconvergente. Finalement on définit pour $X$ quasi-projectif un isomorphisme entre la cohomologie rigide de $X$ et la cohomologie de de Rham-Witt surconvergente rationnelle.

\section{Introduction}

Let $X$ be a smooth variety over a perfect field $k$ of finite characteristic. The purpose of this work is to define an overconvergent de Rham-Witt complex $W^{\dagger} \Omega_{X / k}$ of sheaves on $X$. This complex is a differential graded algebra contained in the de Rham-Witt complex $W \Omega_{X / k}$ of Illusie and Deligne.

If $X$ is quasiprojective we define a canonical isomorphism from rigid cohomology of $X$ in the sense of Berthelot:

$$
H_{\text {rig }}^{i}(X / W(k) \otimes \mathbb{Q}) \rightarrow \mathbb{H}^{i}\left(X, W^{\dagger} \Omega_{X / k}\right) \otimes \mathbb{Q} .
$$


In particular these are finite dimensional vector spaces over $W(k) \otimes \mathbb{Q}$ by [2]. We conjecture that the image of the morphism

$$
\mathbb{H}^{i}\left(X, W^{\dagger} \Omega_{X / k}\right) \rightarrow \mathbb{H}^{i}\left(X, W^{\dagger} \Omega_{X / k}\right) \otimes \mathbb{Q}
$$

is a finitely generated $W(k)$-module. If $X$ is projective we expect that the image of $\mathbb{H}^{i}\left(X, W^{\dagger} \Omega_{X / k}\right)$ under the comparison isomorphism between rigid cohomology and crystalline cohomology coincides with the image of crystalline cohomology.

In the case where $X=\operatorname{Spec} A$ is affine we obtain more precise results. The cohomology groups of the individual sheaves $W^{\dagger} \Omega_{X / k}^{j}$ are zero for $i>0$. The complex $H^{0}\left(X, W^{\dagger} \Omega_{X / k}\right)$ will be denoted by $W^{\dagger} \Omega_{A / k}$. Let $\tilde{A}$ be a lifting of $A$ to a smooth algebra $\tilde{A}$ over $W(k)$. We denote by $\tilde{A}^{\dagger}$ the weak completion of $\tilde{A}$ in the sense of Monsky-Washnitzer. The absolute Frobenius endomorphism on $A$ lifts (non canonically) to $\tilde{A}^{\dagger}$. This defines a homomorphism $\tilde{A}^{\dagger} \rightarrow W(A)$. We show that the image of this map lies in $W^{\dagger}(A)$. This defines morphisms

$$
\text { (1) } H^{i}\left(\Omega_{\tilde{A}^{\dagger} / W(k)}\right) \rightarrow H^{i}\left(W^{\dagger} \Omega_{A / k}^{\cdot}\right), \quad \text { for } i \geq 0 \text {. }
$$

We show that the kernel and cokernel of this map is annihilated by $p^{2 \kappa}$, where $\kappa=\left\lfloor\log _{p} \operatorname{dim} A\right\rfloor$. If we tensor the morphism (1) by $\mathbb{Q}$ it becomes independent of the lift of the absolute Frobenius chosen.

We note that Lubkin [12] used another growth condition on Witt vectors. His bounded Witt vectors are different from our overconvergent Witt vectors.

Let $A=k\left[T_{1}, \ldots, T_{d}\right]$ be the polynomial ring. For each real $\epsilon>0$ we defined ([5]) the Gauss norm $\gamma_{\epsilon}$ on $W(A)$. We extend them to the de Rham-Witt complex $W \Omega_{A / k}$. A Witt differential from $W \Omega_{A / k}$ is called overconvergent if its Gauss norm is finite for some $\epsilon>0$. We denote the subcomplex of all overconvergent Witt differentials by $W^{\dagger} \Omega_{A / k}$. Following the description in [10], $W \Omega_{A / k}$ decomposes canonically into an integral part and an acyclic fractional part and this decomposition continues to hold for the complex of overconvergent Witt differentials. The integral part is easily identified with the de Rham complex associated to the weak completion of the polynomial algebra $W(k)\left[T_{1}, \ldots, T_{d}\right]$ in the sense of Monsky and Washnitzer. This explains the terminology "overconvergent" for Witt differentials. For an arbitrary smooth $k$-algebra $B$ we choose a presentation $A \rightarrow B$. We define the complex of overconvergent Witt differentials $W^{\dagger} \Omega_{B / k}$ as the image of $W^{\dagger} \Omega_{A / k}$. This is independent of the presentation. It is a central result that the functor which associates to a smooth affine scheme $\operatorname{Spec} B$ the group $W^{\dagger} \Omega_{B / k}^{m}$ is a sheaf for the étale topology, and that $H_{\mathrm{Zar}}^{i}\left(\operatorname{Spec} B, W^{\dagger} \Omega_{B / k}^{m}\right)=0$ for $i \geq 1$. For this we generalize ideas of Meredith [13]. One also uses that the ring of overconvergent Witt vectors is weakly complete in the sense of Monsky-Washnitzer [5] and the complex of overconvergent Witt differentials satisfies a similar property of weak completeness. The étale sheaf property depends on an explicit description - for a finite étale extension $C / B$ - of $W^{\dagger} \Omega_{C / k}$ in terms of $W^{\dagger} \Omega_{B / k}$. The result is as nice as one can hope for. By a result of Kedlaya [9] any smooth variety can be covered by affines which are finite étale over a localized polynomial algebra. It then remains to show a localization property of overconvergence; namely a Witt differential of a localized polynomial algebra which becomes overconvergent after further localization is already overconvergent. This requires a detailed study of suitable Gauss norms (that are all equivalent) on the truncated de Rham-Witt complex of a localized polynomial algebra. 
In the final section we globalize the comparison with rigid cohomology from the affine case. In our approach it is essential to use Grosse-Klönne's dagger spaces [6]. Let $Z$ be an affine smooth scheme over $k$. Let $Z \rightarrow F$ be a closed embedding in a smooth affine scheme over $W(k)$. We call $(Z, F)$ a special frame. To a special frame we associate canonically a dagger space $] Z\left[_{\hat{F}}^{\dagger}\right.$. Its de Rham cohomology coincides with the rigid cohomology of $Z$ :

$$
\mathbf{R} \Gamma(] Z\left[_{\hat{F}}^{\dagger}, \Omega_{] Z \Gamma_{\hat{F}}^{\dagger}}\right)=R \Gamma_{\text {rig }}(Z) .
$$

If $F \times$ Spec $W(k)$ Spec $k=Z$ the dagger space $] Z\left[_{\hat{F}}^{\dagger}\right.$ is affinoid. Therefore the hypercohomology is not needed

$$
\Gamma(] Z\left[_{\hat{F}}^{\dagger}, \Omega_{] Z\left[_{\hat{F}}^{\dagger}\right.}^{\dagger}\right)=\mathbf{R} \Gamma(] Z\left[_{\hat{F}}^{\dagger}, \Omega_{] Z\left[_{\hat{F}}^{\dagger}\right.}^{\dagger}\right) .
$$

We show that the latter is true for a big enough class of special frames. Then simplicial methods allow a globalization to the quasiprojective case.

\section{Definition of the overconvergent de Rham-Witt complex}

Let $R$ be an $\mathbb{F}_{p}$-algebra which is an integral domain. We consider the polynomial algebra $A=R\left[T_{1}, \ldots, T_{d}\right]$. Before we recall the de Rham-Witt complex, we review a few properties of the de Rham complex $\Omega_{A / R}$.

There is a natural morphism of graded rings

$$
F: \Omega_{A / R} \rightarrow \Omega_{A / R},
$$

which is the absolute Frobenius on $\Omega_{A / R}^{0}$ and such that ${ }^{F} d T_{i}=T_{i}^{p-1} d T_{i}$. As shown in [10], $\Omega_{A / R}$ has an $R$-basis of so called basic differentials. Their definition depends on certain choices which we will fix now in a more special way than in loc. cit.

We consider functions $k:[1, d] \rightarrow \mathbb{Z}_{\geq 0}$ called weights. On the support $\operatorname{Supp} k=$ $\left\{i_{1}, \ldots, i_{r}\right\}$ we fix an order $i_{1}, \ldots, i_{r}$ with the following properties:

(i) $\operatorname{ord}_{p} k_{i_{1}} \leq \operatorname{ord}_{p} k_{i_{2}} \leq \cdots \leq \operatorname{ord}_{p} k_{i_{r}}$.

(ii) If $\operatorname{ord}_{p} k_{i_{n}}=\operatorname{ord}_{p} k_{i_{n+1}}$, then $i_{n} \leq i_{n+1}$.

Let $\mathscr{P}=\left\{I_{0}, I_{1}, \ldots, I_{l}\right\}$ be a partition of Supp $k$ as in [10]. A basic differential is a differential of the form:

$$
\mathfrak{e}(k, \mathscr{P})=T^{k_{I_{0}}}\left(\frac{d T^{k_{I_{1}}}}{p^{\operatorname{ord}_{p} k_{I_{1}}}}\right) \cdots\left(\frac{d T^{k_{I_{l}}}}{p^{\operatorname{ord}_{p} k_{I_{l}}}}\right) .
$$

It is shown in [10] Proposition 2.1 that the elements (0.1) form a basis of the de Rham complex $\Omega_{A / R}$ as an $R$-module. The de Rham-Witt complex $W \Omega_{A / R}$ has a similar description, but now fractional weight functions are involved. More precisely, an element $\omega \in W \Omega_{A / R}^{r}$ has a unique decomposition as a sum of basic Witt differentials [10]

$$
\omega=\sum_{k, \mathscr{P}} e\left(\xi_{k, \mathscr{D}}, k, \mathscr{P}\right)
$$

where $k:[1, d] \rightarrow \mathbb{Z}_{\geq 0}\left[\frac{1}{p}\right]$ is any weight $([10], 2.2)$ and $\mathscr{P}=\left\{I_{0}, I_{1}, \ldots, I_{r}\right\}$ runs through all partitions of $\operatorname{Supp} k$. Moreover, the coefficients $\xi_{k, \mathscr{P}} \in W(R)$ satisfy a certain convergence condition ([10], Theorem 2.8). 
For each real number $\varepsilon>0$ we define the Gauss norm of $\omega$ :

$$
\gamma_{\varepsilon}(\omega)=\inf _{k, \mathscr{P}}\left\{\operatorname{ord}_{V} \xi_{k, \mathscr{D}}-\varepsilon|k|\right\} .
$$

We will also use the truncated Gauss norms for a natural number $n \geq 0$ :

$$
\gamma_{\varepsilon}[n](\omega)=\inf _{k, \mathscr{P}}\left\{\operatorname{ord}_{V} \xi_{k, \mathscr{P}}-\varepsilon|k| \mid \operatorname{ord}_{V} \xi_{k, \mathscr{P}} \leq n\right\} .
$$

The truncated Gauss norms factor over $W_{n+1} \Omega_{A / R}$. We note that in the truncated case the inf is over a finite set.

If $\gamma_{\varepsilon}(\omega)>-\infty$, we say that $\omega$ has radius of convergence $\varepsilon$.

We call $\omega$ overconvergent, if there is an $\varepsilon>0$ such that $\omega$ has radius of convergence $\varepsilon$. It follows from the definitions that

$$
\gamma_{\varepsilon}\left(\omega_{1}+\omega_{2}\right) \geq \min \left(\gamma_{\varepsilon}\left(\omega_{1}\right), \gamma_{\varepsilon}\left(\omega_{2}\right)\right) .
$$

This inequality shows that the overconvergent Witt differentials form a subgroup of $W \Omega_{A / R}$ which is denoted by $W^{\dagger} \Omega_{A / R}$. We have $W^{\dagger} \Omega_{A / R}=\bigcup_{\varepsilon} W^{\varepsilon} \Omega_{A / R}$ where $W^{\varepsilon} \Omega_{A / R}$ are the overconvergent Witt differentials with radius of convergence $\varepsilon$.

If $\overline{\mathbb{R}}=\mathbb{R} \cup\{\infty\} \cup\{-\infty\}$, then an $\overline{\mathbb{R}}$-valued function $c$ on an abelian group $M$ which satisfies (0.4), so that $c(a+b) \geq \min \{c(a), c(b)\}$, is called an order function.

Definition 0.5. - We say that $\omega$ is homogeneous of weight $k$ if in the sum $\omega=\sum e\left(\xi_{k, \mathscr{D}}, k, \mathscr{P}\right)$ the weight $k$ is fixed. We write weight $(\omega)=k$.

If $g \in \mathbb{Q}$, then we can consider sums which are homogeneous of degree $g$, i.e.

$$
\omega=\sum_{|k|=g, \mathscr{P}} e\left(\xi_{k, \mathscr{P}}, k, \mathscr{P}\right) .
$$

Then we define $\operatorname{deg}(\omega)=g$. If $\omega$ is homogeneous of a fixed degree, we define

$$
\operatorname{ord}_{V} \omega=\min _{\operatorname{ord}} \xi_{k, \mathscr{D}} .
$$

It is easy to see that $\gamma_{\varepsilon}(\omega)>-\infty$ if and only if there are real constants $C_{1}, C_{2}$, with $C_{1}>0$ such that for all weights $k$ occurring in $\omega$ we have

$$
|k| \leq C_{1} \operatorname{ord}_{V} \xi_{k, \mathscr{P}}+C_{2} .
$$

One can take $C_{1}=\frac{1}{\varepsilon}$.

Using this equivalent definition one can show that the product of two overconvergent Witt differentials is again overconvergent, as follows: For two homogeneous forms $\omega_{1}, \omega_{2}$ one has $\operatorname{ord}_{V}\left(\omega_{1} \wedge \omega_{2}\right) \geq \max \left(\operatorname{ord}_{V} \omega_{1}, \operatorname{ord}_{V} \omega_{2}\right)$. This follows from a (rather tedious) case by case calculation with basic Witt differentials.

We have $\operatorname{deg}\left(\omega_{1} \wedge \omega_{2}\right)=\operatorname{deg} \omega_{1}+\operatorname{deg} \omega_{2}$.

Assume now that

$$
\operatorname{deg} \omega \leq C_{1} \operatorname{ord}_{V} \omega+C_{2}
$$

and

$$
\operatorname{deg} \omega^{\prime} \leq C_{1}^{\prime} \operatorname{ord}_{V} \omega^{\prime}+C_{2}^{\prime}
$$

for two homogeneous forms $\omega, \omega^{\prime}$ of fixed degrees. Then

$$
\operatorname{deg}\left(\omega \wedge \omega^{\prime}\right)=\operatorname{deg} \omega+\operatorname{deg} \omega^{\prime} \leq\left(C_{1}+C_{1}^{\prime}\right) \operatorname{ord}_{V}\left(\omega_{1} \wedge \omega_{2}\right)+C_{2}+C_{2}^{\prime} .
$$

4 e SÉRIE - TOME $44-2011$ - No 2 
This implies that if $\omega$ and $\omega^{\prime}$ are overconvergent Witt differentials with radii of convergence $\varepsilon$ and $\varepsilon^{\prime}$ then $\omega \wedge \omega^{\prime}$ is overconvergent with radius of convergence $\frac{\varepsilon \cdot \varepsilon^{\prime}}{\varepsilon+\varepsilon^{\prime}}$. In the special case $\varepsilon=\varepsilon^{\prime}$ we get that $\omega \wedge \omega^{\prime}$ is overconvergent with radius of convergence $\frac{\varepsilon}{2}$ and $\gamma_{\frac{\varepsilon}{2}}\left(\omega \wedge \omega^{\prime}\right) \geq \frac{\gamma_{\varepsilon}(\omega)+\gamma_{\varepsilon}\left(\omega^{\prime}\right)}{2}$. This shows that $W^{\dagger} \Omega_{A / R}$ is a differential graded algebra over the ring $W^{\dagger}(A)$ of overconvergent Witt vectors.

We recall from [5] the definition of a pseudovaluation. An order function $c$ on a ring $M$ is called a pseudovaluation if in addition it satisfies: (i) $c(1)=0$ and $c(0)=\infty$; (ii) $c(m)=c(-m)$ for all $m \in M$; (iii) $c\left(m_{1} m_{2}\right) \geq c\left(m_{1}\right)+c\left(m_{2}\right)$ if $c\left(m_{1}\right) \neq-\infty$, $c\left(m_{2}\right) \neq-\infty$.

In general, the Gauss norms $\gamma_{\epsilon}$ form a set of pseudovaluations on the ring of Witt vectors, i.e. in degree zero; however, from the formula ${ }^{V}\left[T^{p-1}\right] d^{V}[T]=p d[T]$ and

$$
\operatorname{ord}_{V}\left({ }^{V}\left[T^{p-1}\right]\right)=\operatorname{ord}_{V}\left(d^{V}[T]\right)=\operatorname{ord}_{V}(p d[T])=1,
$$

we see that we cannot expect a formula

$$
\gamma_{\varepsilon}\left(\omega_{1} \wedge \omega_{2}\right) \geq \gamma_{\varepsilon}\left(\omega_{1}\right)+\gamma_{\varepsilon}\left(\omega_{2}\right)
$$

Hence the Gauss norms do not extend to pseudovaluations in higher degrees.

Proposition 0.7. - Let $R$ be an integral domain such that $p \cdot R=0 . \quad$ Let $\varphi: R\left[T_{1}, \ldots, T_{d}\right] \rightarrow R\left[U_{1}, \ldots, U_{l}\right]$ be a homomorphism. It induces a map

$$
\varphi_{*}: W \Omega_{R\left[T_{1}, \ldots, T_{d}\right] / R} \rightarrow W \Omega_{R\left[U_{1}, \ldots, U_{l}\right] / R} .
$$

Then there is a constant $\alpha>0$, such that for any $\varepsilon>0$ and any natural number $n$ :

$$
\gamma_{\alpha \varepsilon}[n]\left(\varphi_{*} \omega\right) \geq \gamma_{\varepsilon}[n](\omega)
$$

The same inequality holds if $[n]$ is removed. In particular, if $\omega$ is overconvergent with radius of convergence $\varepsilon$ then $\varphi_{*} \omega$ is overconvergent with radius of convergence $\alpha \varepsilon$.

Proof. - We set $Y_{j}=\left[U_{j}\right]$ and $X_{i}=\left[T_{i}\right]$. From Lemma 2.23 in [5] we obtain an expansion:

$$
\varphi_{*}\left(X_{i}\right)=\left[Q_{i}\left(U_{1}, \ldots, U_{l}\right)\right]=\sum_{|k|<c} a_{i k} Y^{k},
$$

where $a_{i k} \in W(R)$. More generally we obtain for a monomial $X^{l}=X_{1}^{l_{1}} \ldots X_{d}^{l_{d}}, l_{i} \in \mathbb{Z}_{\geq 0}$ an expansion:

$$
\varphi_{*}\left(X^{l}\right)=\sum_{|k|<c|l|} b_{k} Y^{k}, \quad b_{k} \in W(R) .
$$

Since $\varphi_{*}$ commutes with the action of $V$ we find for $l$ not necessarily integral

$$
\begin{aligned}
\varphi_{*}\left(V^{u} \eta X^{l}\right) & ={ }^{u}\left(\varphi_{*}\left(\eta X^{l \cdot p^{u}}\right)\right) \\
& =\left(\sum_{k^{\prime} \leq c|l| p^{u}} \eta \cdot b_{k^{\prime}} \cdot Y^{k^{\prime}}\right)=\sum_{|k|<c \cdot|l|} V^{u}\left(\eta b_{k^{\prime}}\right) \cdot Y^{k} .
\end{aligned}
$$

From this we see immediately the following fact: Let $\omega \in W \Omega_{R\left[T_{1}, \ldots, T_{d}\right] / R}$ be a Witt differential which is homogeneous of degree $l$, and such that $\operatorname{ord}_{V} \omega=m$. Then $\varphi_{*} \omega$ is a 
$V$-convergent sum $\sum \eta_{k}$ of homogeneous Witt differentials of degree $|k|<c|l|$ and such that $\operatorname{ord}_{V} \eta_{k} \geq m$. Assume that $\omega=\sum \omega_{l}$ is a sum of homogeneous differentials such that

$$
\operatorname{ord}_{V} \omega_{l}-\varepsilon|l| \geq D \text {. }
$$

Then $\varphi_{*} \omega_{l}=\sum \eta_{l, k}$, where $\eta_{l, k}$ is homogeneous of degree $k$, such that $|k| \leq c|l|$ and $\operatorname{ord}_{V} \eta_{l, k} \geq m$. Therefore for $\delta>0$,

$$
\operatorname{ord}_{V} \eta_{l, k}-\delta|k| \geq m-\delta c|l| \text {. }
$$

If $\delta<\frac{\varepsilon}{c}$ the last expression is bounded below by $D$. This proves the proposition with $\alpha=1 / c$.

By the proposition we obtain a map:

$$
W^{\varepsilon} \Omega_{R\left[T_{1}, \ldots, T_{d}\right] / R} \rightarrow W^{\alpha \varepsilon} \Omega_{R\left[U_{1}, \ldots, U_{l}\right] / R} .
$$

Proposition 0.9. - Let $\varphi: R\left[T_{1}, \ldots, T_{d}\right] \longrightarrow R\left[U_{1}, \ldots, U_{l}\right]$ be an $R$-algebra homomorphism. Then the induced map

$$
\varphi_{*}: W \Omega_{R\left[T_{1}, \ldots, T_{d}\right] / R} \rightarrow W \Omega_{R\left[U_{1}, \ldots, U_{l}\right] / R}
$$

maps $W^{\dagger} \Omega_{R\left[T_{1}, \ldots, T_{d}\right] / R}$ to $W^{\dagger} \Omega_{R\left[U_{1}, \ldots, U_{l}\right] / R}$.

If, moreover, $\varphi$ is surjective then

$$
W^{\dagger} \Omega_{R\left[T_{1}, \ldots, T_{d}\right] / R} \rightarrow W^{\dagger} \Omega_{R\left[U_{1}, \ldots, U_{l}\right] / R}
$$

is surjective too.

Proof. - Only the last statement needs a verification. If $\varphi$ is surjective we find a homomorphism

$$
\psi: R\left[U_{1}, \ldots, U_{l}\right] \rightarrow R\left[T_{1}, \ldots, T_{d}\right],
$$

such that $\varphi \circ \psi=i d$. Then for $\eta \in W^{\dagger} \Omega_{R\left[U_{1}, \ldots, U_{l}\right] / R}, \psi \eta$ is overconvergent and therefore a preimage of $\eta$.

We have seen that $\gamma_{\varepsilon}$ fails to be a pseudovaluation on the ring $W \Omega_{A / R}$. However we will face a situation where we will need an inequality

$$
\gamma_{\varepsilon}(f \omega) \geq \gamma_{\varepsilon}(f)+\gamma_{\varepsilon}(\omega)
$$

for certain $f \in W(A)$ and $\omega \in W \Omega_{A / R}$. For suitable $f$ and overconvergent $\omega$ we can even achieve equality.

From now on, let $R=k$ be a perfect field. Let $A=k\left[T_{1}, \ldots, T_{d}\right]$ be the polynomial ring. The Teichmüller of $T_{i}$ in $W(A)$ is denoted by $X_{i}$. For a Witt differential $\omega \in W \Omega_{A / k}$ we define:

$$
\nu_{p}(\omega)=\max \left\{a \in \mathbb{Z} \mid p^{-a} \omega \in W \Omega_{A / k}\right\} .
$$

Obviously we have that

$$
\nu_{p}\left(\omega_{1} \omega_{2}\right) \geq \nu_{p}\left(\omega_{1}\right)+\nu_{p}\left(\omega_{2}\right)
$$

for arbitrary Witt differentials.

Let $\omega=\mathbf{e}(\xi, k, \mathscr{P})$ be a basic Witt differential. Let $p^{u}$ be the denominator of the weight $k$. Then we have:

$$
\operatorname{ord}_{V} \omega=\operatorname{ord}_{V} \xi=\nu_{p}(\omega)+u
$$

$4{ }^{\text {e }}$ SÉRIE - TOME $44-2011-\mathrm{N}^{\circ} 2$ 
For an arbitrary $\omega \in W \Omega_{A / k}$ we write the expansion:

$$
\omega=\sum_{k, \mathscr{D}} \mathbf{e}\left(\xi_{k, \mathscr{P}}, k, \mathscr{P}\right)
$$

Let $\varepsilon>0$. We have the Gauss norm $\gamma_{\varepsilon}$ :

$$
\gamma_{\varepsilon}(\omega)=\inf _{k, \mathscr{P}}\left\{\operatorname{ord}_{V}\left(\mathbf{e}\left(\xi_{k, \mathscr{D}}, k, \mathscr{P}\right)\right)-\varepsilon|k|\right\}
$$

We also define the modified Gauss norm:

$$
\breve{\gamma}_{\varepsilon}(\omega)=\inf _{k, \mathscr{D}}\left\{\nu_{p}\left(\mathbf{e}\left(\xi_{k, \mathscr{D}}, k, \mathscr{P}\right)\right)-\varepsilon|k|\right\}
$$

We note that:

$$
\gamma_{\varepsilon}(\omega) \geq \breve{\gamma}_{\varepsilon}(\omega)
$$

Consider the polynomial algebra $\tilde{A}=W(k)\left[X_{1}, \ldots, X_{d}\right]$. For each real number $\varepsilon>0$ we define on $\tilde{A}$ a valuation $\gamma_{\varepsilon}$. We write $f \in \tilde{A}$. We will use the vector notation $I=\left(i_{1}, \ldots, i_{d}\right)$ and write

$$
f=\sum c_{I} \underline{X}^{I}, \quad c_{I} \in W(k)
$$

We write $|I|=i_{1}+\cdots+i_{d}$. Then we set

$$
\left.\gamma_{\varepsilon}(f)=\min \left\{\operatorname{ord}_{p}\left(c_{I}\right)-\varepsilon|I|\right)\right\}
$$

We extend $\gamma_{\varepsilon}$ to the differential forms $\Omega_{\tilde{A} / W(k)}$. We write a differential form as of degree $r$ :

$$
\omega=\sum_{\alpha} f_{\alpha} d X_{\alpha_{1}} \wedge \cdots \wedge d X_{\alpha_{r}}, \quad f_{\alpha} \in \tilde{A}
$$

where $\alpha=\left(\alpha_{1}, \ldots, \alpha_{r}\right)$ runs over vectors with $1 \leq \alpha_{1}<\cdots<\alpha_{r} \leq d$. Then we set:

$$
\gamma_{\varepsilon}(\omega)=\min _{\alpha}\left\{\gamma_{\varepsilon}\left(f_{\alpha}\right)-r \varepsilon\right\}
$$

We have the following properties:

$$
\begin{aligned}
& \gamma_{\varepsilon}(f \omega) \quad=\gamma_{\varepsilon}(f)+\gamma_{\varepsilon}(\omega), \quad f \in \tilde{A} \\
& \gamma_{\varepsilon}\left(\omega_{1} \wedge \omega_{2}\right) \geq \gamma_{\varepsilon}\left(\omega_{1}\right)+\gamma_{\varepsilon}\left(\omega_{2}\right), \omega_{i} \in \Omega_{\tilde{A} / W(k)} .
\end{aligned}
$$

We may write $\omega$ as a sum of $p$-basic elements [10] (2.3):

$$
\mathbf{e}(c, k, \mathscr{P})=c X^{k_{I_{0}}} \frac{d X^{k_{I_{1}}}}{p^{\operatorname{ord}_{p} k_{I_{1}}}} \cdots \frac{d X^{k_{I_{l}}}}{p^{\operatorname{ord}_{p} k_{I_{l}}}} .
$$

Lemma 0.13. - Let us write $\omega \in \Omega_{\tilde{A} / W(k)}$ as a sum of p-basic differentials:

$$
\omega=\sum \mathbf{e}\left(c_{k, \mathscr{D}}, k, \mathscr{P}\right) \text {. }
$$

Then we have:

$$
\gamma_{\varepsilon}(\omega)=\min \left\{\operatorname{ord}_{p}\left(c_{k, \mathscr{P}}\right)-|k| \varepsilon\right\} .
$$

Proof. - Clearly it is sufficient to consider the case where $\omega$ belongs to the free $W(k)$-module of forms of a given weight $k$ (compare [10] proof of Prop. 2.1). Then $\omega$ may be written:

$$
\omega=\sum b_{i_{1} \ldots i_{l}} X_{1}^{k_{1}} \cdots X_{n}^{k_{n}} d \log X_{i_{1}} \wedge \cdots \wedge d \log X_{i_{l}} .
$$

The result follows because $b_{i_{1} \ldots i_{l}}$ and $c_{k, \mathscr{D}}$ are related by a unimodular matrix with coefficients in $\mathbb{Z}_{p}$, [10] 2.1. 
Consider the natural map $\tilde{A} \rightarrow W(A)$ which sends $X_{i}$ to the Teichmüller representative $\left[T_{i}\right]$. It induces a map:

$$
\Omega_{\tilde{A} / W(k)} \rightarrow W \Omega_{A / k} .
$$

The $p$-adic completion of the image of this map consists of the integral Witt differentials. From Lemma 0.13 we obtain:

Proposition 0.15. - The map (0.14) is compatible with the Gauss norms $\gamma_{\varepsilon}$ on both sides.

Corollary 0.16. - Let $\omega, \eta \in W \Omega_{A / k}$. Then we have:

$$
\begin{aligned}
& \gamma_{\varepsilon}(\omega \eta) \geq \gamma_{\varepsilon}(\omega)+\gamma_{\varepsilon}(\eta) \text { for } \omega \text { integral } \\
& \gamma_{\varepsilon}(\omega \eta) \geq \breve{\gamma}_{\varepsilon}(\omega)+\gamma_{\varepsilon}(\eta) \text { for } \omega \text { arbitrary. }
\end{aligned}
$$

We note that for $\omega$ integral, $\gamma_{\varepsilon}(\omega)=\breve{\gamma}_{\varepsilon}(\omega)$. Let $f \in A$, then we have $\breve{\gamma}_{\varepsilon}([f])=\gamma_{\varepsilon}([f])$. In particular we find for arbitrary $\omega$

$$
\gamma_{\varepsilon}([f] \omega) \geq \gamma_{\varepsilon}([f])+\gamma_{\varepsilon}(\omega)
$$

Proof. - We begin with the first inequality. If $\eta$ is integral too, we can apply (0.12). For the general case we may assume that $\eta={ }^{u} \tau$ or $\eta=d^{V^{u}} \tau$ where $\tau$ is a primitive basic Witt differential. We note that for primitive $\tau$ :

$$
\gamma_{\varepsilon}\left(V^{u} \tau\right)=u+\gamma_{\varepsilon / p^{u}}(\tau)
$$

For integral $\omega$ we have

$$
\gamma_{\varepsilon / p^{u}}\left(F^{u} \omega\right)=\gamma_{\varepsilon}(\omega)
$$

If $\omega$ is not integral we have only the inequality:

$$
\gamma_{\varepsilon / p^{u}}\left(F^{u} \omega\right) \geq \gamma_{\varepsilon}(\omega)-u
$$

Then we find using the integral case:

$$
\begin{aligned}
\gamma_{\varepsilon}\left(\omega^{V^{u}} \tau\right) & =\gamma_{\varepsilon}\left({ }^{V^{u}}\left({ }^{F^{u}} \omega \tau\right)\right) \geq u+\gamma_{\varepsilon / p^{u}}\left({ }^{F^{u}} \omega \tau\right) \\
& \geq u+\gamma_{\varepsilon / p^{u}}\left({ }^{F^{u}} \omega\right)+\gamma_{\varepsilon / p^{u}}(\tau)=\gamma_{\varepsilon}\left({ }^{V^{u}} \tau\right)+\gamma_{\varepsilon / p^{u}}\left({ }^{F^{u}} \omega\right) \geq \gamma_{\varepsilon}\left({ }^{V^{u}} \tau\right)+\gamma_{\varepsilon}(\omega)
\end{aligned}
$$

The case $\eta=d^{V^{u}} \tau$ is reduced to the former case by the Leibniz rule:

$$
\omega d^{V^{u}} \tau=d\left(\omega^{V^{u}} \tau\right)-(d \omega)^{V^{u}} \tau
$$

Now we verify the second inequality. We may assume that $\omega={ }^{u} \tau$ or $\omega=d^{V^{u}} \tau$ for a primitive basic Witt differential. Then we have:

$$
\begin{aligned}
\breve{\gamma}_{\varepsilon}(\omega) & =\gamma_{\varepsilon / p^{u}}(\tau), \text { and } \\
\gamma_{\varepsilon}\left({ }^{V^{u}} \tau \eta\right) & =\gamma_{\varepsilon}\left({ }^{V^{u}}\left(\tau^{F^{u}} \eta\right)\right) \geq u+\gamma_{\varepsilon / p^{u}}\left(\tau^{F^{u}} \eta\right) \\
& \geq u+\gamma_{\varepsilon / p^{u}}(\tau)+\gamma_{\varepsilon}\left(F^{u} \eta\right)=\breve{\gamma}_{\varepsilon}(\omega)+u+\gamma_{\varepsilon / p^{u}}\left(F^{u} \eta\right) \geq \breve{\gamma}_{\varepsilon}(\omega)+\gamma_{\varepsilon}(\eta) .
\end{aligned}
$$

Finally we have to show that $\gamma_{\varepsilon}([f])=\breve{\gamma}_{\varepsilon}([f])$. We denote by $m=\left(m_{1}, \ldots, m_{d}\right)$ a vector of non negative integers and write:

$$
f=\sum_{m} a_{m} T_{1}^{m_{1}} \cdots T_{d}^{m_{d}}=\sum_{m} a_{m} \underline{T}^{m} .
$$


Let $g$ be the total degree of $f$. Then we have

$$
\gamma_{\varepsilon}([f])=-\varepsilon g .
$$

We enumerate the $m$ with $a_{m} \neq 0$ :

$$
m(1), \ldots, m(t)
$$

By Lemma 2.23 in [5] we find:

$$
[f]=\sum_{k_{1}+\cdots+k_{t}=1} \alpha_{k_{1}, \ldots, k_{t}}[\underline{T}]^{m(1) k_{1}+\cdots+m(t) k_{t}} .
$$

If we take $\breve{\gamma}_{\varepsilon}$ of one summand it is bigger than the degree of this summand times $-\varepsilon$ :

$$
\begin{aligned}
\breve{\gamma}_{\varepsilon}\left(\alpha_{k_{1}, \ldots, k_{t}}[\underline{T}]^{m(1) k_{1}+\cdots+m(t) k_{t}}\right) & \geq-\varepsilon\left(|m(1)| k_{1}+\cdots+|m(t)| k_{t}\right) \\
& \geq-\varepsilon\left(g k_{1}+\cdots+g k_{t}\right)=-\varepsilon g .
\end{aligned}
$$

This shows that $\breve{\gamma}_{\varepsilon}([f]) \geq-\varepsilon g=\gamma_{\varepsilon}([f])$. The other inequality is obvious.

Proposition 0.19. - Let $f \in W(A), f=\left(f_{0}, f_{1}, \ldots\right)$ be a Witt vector, such that $f_{0} \neq 0$. Let $\omega \in W \Omega_{A / k}$ be an element, whose decomposition into basic Witt differentials has the following form:

$$
\omega=\sum \mathbf{e}\left(\xi_{k, \mathscr{D}}, k, \mathscr{P}\right)
$$

We assume that all weights $k$ appearing in this decomposition have the same denominator $p^{u}$ with $u \geq 0$, and the same degree $\kappa=|k|$. Moreover we assume that only partitions $\mathscr{P}$ with $I_{0} \neq \varnothing$ appear and that there are a weight $k$ and a partition $\mathscr{D}$ such that $\operatorname{ord}_{V} \xi_{k, \mathscr{P}}=u$. The last condition says that there are $k$ and $\mathscr{P}$ such that $\mathbf{e}\left(\xi_{k, \mathscr{P}}, k, \mathscr{P}\right)={ }^{u} \tau$, for a primitive basic Witt differential $\tau$.

We can write $f \omega$ as a sum of basic Witt differentials:

$$
f \omega=\sum \mathbf{e}\left(\xi_{h, \mathscr{P}}^{\prime}, h, \mathscr{P}\right) .
$$

Then there is a summand $\mathbf{e}\left(\xi_{h, \mathscr{P}}^{\prime}, h, \mathscr{P}\right)$ such that $\operatorname{ord}_{V}\left(\xi_{h, \mathscr{P}}^{\prime}\right)=u$, such that h has denominator $p^{u}$, and such that $I_{0} \neq \varnothing$. Moreover if $g$ is the degree of the polynomial $f_{0}$, then the degree of $h$ is $|h|=g+\kappa$.

In particular we have the inequality:

$$
\gamma_{\varepsilon}(f \omega) \leq \gamma_{\varepsilon}(\omega)-\varepsilon \operatorname{deg} f_{0}
$$

Proof. - We write:

$$
f=\tilde{f}+{ }^{V} \rho,
$$

where $\tilde{f}$ is a polynomial in $X_{1}=\left[T_{1}\right], \ldots, X_{d}=\left[T_{d}\right]$ with coefficients in $W(k)$, which are not divisible by $p$. The degrees of the polynomials $f_{0}$ and $\tilde{f}$ are the same.

We set $\omega=V^{u} \tau$, where $\tau$ is an integral Witt differential with $\nu_{p}(\tau)=1$. Then we have:

$$
f \omega=\left(\tilde{f}+{ }^{V} \rho\right)^{V^{u}} \tau={ }^{u}\left(F^{u} \tilde{f}+p^{F^{u-1}} \rho \tau\right) .
$$

We write $\tilde{f}=\sum_{i} \tilde{f}_{i}$ as a sum of homogeneous polynomials of different degree $g_{i}$. The maximum of the $g_{i}$ is $g$. Then the Witt differential $\eta_{i}=F^{u} \tilde{f}_{i} \tau$ is for each $i$ an integral homogeneous Witt differential of degree $p^{u} g_{i}+p^{u} \kappa$. By assumption the reduction of this Witt differential in $\Omega_{A / k}$ is not closed. The basic Witt differentials which appear in the 
decomposition of $\eta_{i}$ have weights which are not divisible by $p$, because the weights appearing in $F^{u} \tilde{f}$ are divisible by $p$ but those appearing in $\tau$ are not divisible by $p$. This shows that primitive basic Witt differentials appear in the decomposition of each $\eta_{i}$. These cannot be destroyed by basic Witt differentials which appear in the decomposition of the last summand in the brackets of (0.22), because of the factor $p$. If we apply $V^{u}$ we obtain the desired basic Witt differential in the decomposition of $f \omega$.

COROLlary 0.23. - With the notations of the proposition consider a Witt differential of the form $\omega_{1}=\omega+d \eta$, and write

$$
f^{p} \omega_{1}=\sum \mathbf{e}\left(\hat{\xi}_{\hat{h}, \mathscr{D}}, \hat{h}, \mathscr{P}\right) .
$$

Then there is a summand $\mathbf{e}\left(\hat{\xi}_{\hat{h}, \mathscr{D}}, \hat{h}, \mathscr{P}\right)$ in the above sum, such that $\operatorname{ord}_{V} \hat{\xi}_{\hat{h}, \mathscr{D}}=u$, such that $\hat{h}$ has denominator $p^{u}$ and such that $I_{0} \neq \varnothing$. Moreover the degree of $\hat{h}$ is $|\hat{h}|=p g+\kappa$.

Proposition 0.24. - Let $f_{0} \in A=k\left[T_{1}, \ldots, T_{d}\right]$ be a polynomial of degree $g$. Let $\omega \in W \Omega_{L / k}$. Then we have for the Gauss norm on $A$ :

$$
\gamma_{\varepsilon}\left(\left[f_{0}\right] \omega\right)=\gamma_{\varepsilon}\left(\left[f_{0}\right]\right)+\gamma_{\varepsilon}(\omega) .
$$

Proof. - We write $\omega$ as a sum of basic Witt differentials:

$$
\omega=\sum_{i \in I} e_{i}
$$

By continuity we may assume that the sum is finite. By Corollary 0.16 we have the inequality:

$$
\gamma_{\varepsilon}\left(\left[f_{0}\right] \omega\right) \geq \gamma_{\varepsilon}\left(\left[f_{0}\right]\right)+\gamma_{\varepsilon}(\omega) .
$$

We may therefore assume that in the sum (0.26)

$$
\gamma_{\varepsilon}(\omega)=\gamma_{\varepsilon}\left(e_{i}\right)
$$

for all $i \in I$. We may further assume that $\nu_{p}(\omega)=0$.

Let us first consider the case where there is an integral basic Witt differential $e_{i_{0}}$ in the sum (0.26) such that $\nu_{p}\left(e_{i_{0}}\right)=0$. Then we decompose $\omega$ into three parts:

$$
\omega=\eta+\omega^{\prime}+\omega^{\prime \prime},
$$

where $\eta$ is the sum of those Witt differentials $e_{i}$ in (0.26) which are integral and such that $\nu_{p}\left(e_{i}\right)=0$, where $\omega^{\prime}$ is the sum of those Witt differentials $e_{i}$ in (0.26) which are integral and such that $\nu_{p}\left(e_{i}\right)>0$, and where $\omega^{\prime \prime}$ is the sum of those Witt differentials in (0.26) which are not integral.

Let $e_{i}$ be a summand in $\eta$ and let $\kappa$ be its degree. By assumption we find:

$$
\gamma_{\varepsilon}(\omega)=\gamma_{\varepsilon}\left(e_{i}\right)=\nu_{p}\left(e_{i}\right)-\varepsilon \kappa=-\varepsilon \kappa .
$$

It follows that all these $e_{i}$ have the same degree $\kappa$.

Consider the differential $f_{0} \bar{\eta} \in \Omega_{A / k}$ which is the reduction of $\left[f_{0}\right] \eta$. If we write the reduction as a sum of basic differentials in $\Omega_{A / k}$ it must clearly contain a basic Witt differential of degree $g+\kappa$. In the decomposition of $\left[f_{0}\right] \eta$ appears therefore an integral basic Witt differential $\breve{e}$ of degree $g+\kappa$ such that $\nu_{p}(\breve{e})=0$. On the other hand all basic Witt differentials which appear in the decomposition of $\left[f_{0}\right]\left(\omega^{\prime}+\omega^{\prime \prime}\right) \in V W \Omega_{A / k}+d V W \Omega_{A / k}$ are either integral with $\nu_{p}>0$ or nonintegral. Therefore they cannot destroy completely $\breve{e}$. 
We found in the decomposition of $\left[f_{0}\right] \omega$ an integral basic Witt differential $\breve{e}^{\prime}$ of degree $g+\kappa$, such that $\nu_{p}\left(\breve{e}^{\prime}\right)=0$. We conclude that

$$
\gamma_{\varepsilon}\left(\left[f_{0}\right] \omega\right) \leq \gamma_{\varepsilon}\left(\breve{e^{\prime}}\right)=-\varepsilon(g+\kappa)=\gamma_{\varepsilon}\left(\left[f_{0}\right]\right)+\gamma_{\varepsilon}(\omega) .
$$

Since we know the opposite inequality we obtain the Equation (0.25) in the first case.

Let $\omega$ be a Witt differential which does not belong to the first case. Then we write:

$$
\omega=\omega(u)+\omega(d u)+\omega^{\prime}+\omega^{\prime \prime}
$$

where $\omega^{\prime}$ is the sum of all $e_{i}$ in $(0.26)$, such that $\nu_{p}\left(e_{i}\right)>0$. There is a natural number $u$ such that the following holds:

$$
\omega^{\prime \prime} \in V^{u+1} W \Omega_{A / k}+d V^{u+1} W \Omega_{A / k}
$$

and each basic Witt differential appearing in the decomposition of $\omega(u)$ is of the form ${ }^{u} \tau$ for a primitive basic Witt differential $\tau$ and any basic Witt differential which appears in $\omega(d u)$ is of the form $d^{V^{u}} \tau$. By our assumption (0.28) we find that for each of these $\tau$ :

$$
\gamma_{\varepsilon}(\omega)=u+\gamma_{\varepsilon / p^{u}}(\tau)=u-\varepsilon \kappa,
$$

where $\kappa$ is obviously independent of $\tau$.

Before proceeding we make a general remark: It suffices to show the equality $(0.25)$ in the case where $f_{0}$ is a $p$-th power $f_{0}=g_{0}^{p}$. Indeed assuming this we have for arbitrary $f_{0}$ :

$$
\gamma_{\varepsilon}\left(\left[f_{0}^{p}\right] \omega\right)=\gamma_{\varepsilon}\left(\left[f_{0}^{p}\right]\right)+\gamma_{\varepsilon}(\omega)=p \gamma_{\varepsilon}\left(\left[f_{0}\right]+\gamma_{\varepsilon}(\omega) .\right.
$$

On the other hand we already know the inequality:

$$
\gamma_{\varepsilon}\left(\left[f_{0}^{p}\right] \omega\right) \geq(p-1) \gamma_{\varepsilon}\left(\left[f_{0}\right]\right)+\gamma_{\varepsilon}\left(\left[f_{0}\right] \omega\right) .
$$

We conclude:

$$
\gamma_{\varepsilon}\left(\left[f_{0}\right]\right) \gamma_{\varepsilon}(\omega) \geq \gamma_{\varepsilon}\left(\left[f_{0}\right] \omega\right)
$$

Since we already know the opposite the inequality $(0.25)$ follows.

We consider now the second case where $\omega(u) \neq 0$. By Proposition 0.19 the product $\left[f_{0}\right] \omega(u)$ contains a basic Witt differential $e(\xi, k, \mathscr{P})$, where $k$ is a weight of denominator $u>0$, such that $|k|=g+\kappa$ and $\operatorname{ord}_{V} \xi=u$. This basic Witt differential cannot be destroyed by any basic Witt differential appearing in $\left[f_{0}\right] \omega^{\prime}$, because $\nu_{p}>0$, or by any basic Witt differential appearing in $\left[f_{0}\right] \omega^{\prime \prime}$, because those have reduction 0 in $W_{u} \Omega_{A} / k$. It can also not cancel with an exact basic Witt differential appearing in $\left[f_{0}\right] \omega(d u)$. Indeed since $f_{0}$ is a $p$-th power those basic Witt differentials are either exact or have $\nu_{p}>0$. Therefore $\left[f_{0}\right] \omega$ contains as a summand a basic Witt differential $e(\eta, k, \mathscr{P})$ where $k$ is a weight of denominator $u>0$, such that $|k|=g+\kappa$ and $\operatorname{ord}_{V} \xi=u$. This proves the inequality:

$$
\gamma_{\varepsilon}\left(\left[f_{0}\right] \omega\right) \leq u-\varepsilon(g+\kappa)=\gamma_{\varepsilon}\left(\left[f_{0}\right]\right)+\gamma_{\varepsilon}(\omega) .
$$

This gives the desired equality in the second case.

Let us now consider the third and last case, where $\omega(u)=0$ in $(0.29)$. Then we rewrite (0.29) in the form:

$$
\omega=d^{V^{u}} \sigma+\omega^{\prime}+\omega^{\prime \prime}
$$


where $\sigma$ is a sum of primitive basic Witt differentials of the same degree $p^{u} \kappa$, where $\gamma_{\varepsilon}(\omega)=u-\varepsilon \kappa$. We assume as above that $f_{0}=g_{0}^{p}$. We find:

$$
\left[f_{0}\right] d^{V^{u}} \sigma=d\left(\left[g_{0}^{p}\right]^{V^{u}} \sigma\right)-p\left[h_{0}\right]^{p-1}\left(d\left[h_{0}\right]\right)^{V^{u}} \sigma .
$$

By Proposition 0.19 we know that $\left[h_{0}^{p}\right]^{V^{u}} \sigma$ contains a non-closed basic Witt differential

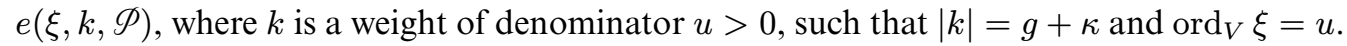
As before we see that the basic Witt differential $\operatorname{de}(\xi, k, \mathscr{P})$ cannot be destroyed by any basic Witt differential which appears in $\left[f_{0}\right] \omega^{\prime}$ or $\left[f_{0}\right] \omega^{\prime \prime}$. It cannot also be destroyed by a basic Witt differential which appears in the last summand of $(0.31)$, because for them $\nu_{p}$ is positive. From this we conclude as before the desired equality $(0.25)$.

Corollary 0.32. - Let $\tilde{f} \in W\left(k\left[T_{1}, \ldots, T_{d}\right]\right)=W(A)$ be an integral Witt vector with radius of convergence $\varepsilon$. Let $\omega \in W \Omega_{A / k}$ be an arbitrary Witt differential of radius of convergence $\varepsilon$. Then we have:

$$
\gamma_{\varepsilon}(\tilde{f} \omega)=\gamma_{\varepsilon}(\tilde{f})+\gamma_{\varepsilon}(\omega)
$$

Proof. - By Corollary 0.16 we have the inequality:

$$
\gamma_{\varepsilon}(\tilde{f} \omega) \geq \gamma_{\varepsilon}(\tilde{f})+\gamma_{\varepsilon}(\omega)
$$

For the opposite inequality we may assume that $\tilde{f}$ is a polynomial by considering the truncations in $W_{n} \Omega_{A / k}$. We write $\tilde{f}=\sum_{i} \tilde{f}_{i}$ as a sum of homogeneous polynomials $\tilde{f}_{i}$ of different degrees $g_{i}$. By the inequality (0.33) we may assume that $\gamma_{\varepsilon}(\tilde{f})=\gamma_{\varepsilon}\left(\tilde{f}_{i}\right)$ for each $i$. Moreover we may clearly assume that $\nu_{p}(\tilde{f})=0$. With these remarks the proof works in the same way as above.

\section{Sheaf properties of the overconvergent de Rham-Witt complex}

Let $A=k\left[t_{1}, \ldots, t_{r}\right]$ be a smooth finitely generated $k$-algebra, $S=k\left[T_{1}, \ldots, T_{r}\right]$ a polynomial algebra. Then $S \rightarrow A, T_{i} \rightarrow t_{i}$ induces a canonical epimorphism

$$
\lambda: W \Omega_{S / k}^{\bullet} \rightarrow W \Omega_{A / k}^{\bullet}
$$

of de Rham-Witt complexes.

Definition 1.1. - We set $W^{\dagger} \Omega_{A / k}^{\bullet}=$ image $\left(W^{\dagger} \Omega_{S / k}^{\bullet}\right)$ under $\lambda$.

We have seen in Proposition 0.9 that this definition is independent from the choice of generators and the representation $S \rightarrow A$. The same proposition shows that the assignment $A \mapsto W^{\dagger} \Omega_{A / k}$ is functorial. Indeed, given smooth finitely generated $k$-algebras $A, B$ as above, and a presentation $k\left[T_{1}, \ldots, T_{r}\right] \rightarrow A$, we extend this to a presentation $k\left[T_{1}, \ldots, T_{r}, U_{1}, \ldots, U_{l}\right] \rightarrow B$ such that the following diagram commutes:

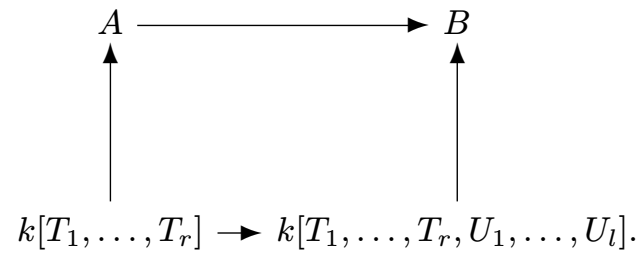

Then it is clear that the induced map $W \Omega_{A / k} \rightarrow W \Omega_{B / k}$ sends $W^{\dagger} \Omega_{A / k} \rightarrow W^{\dagger} \Omega_{B / k}$. 
For $\omega \in W \Omega_{A / k}^{\bullet}$ a convergent sum of images of basic Witt differentials in $W \Omega_{S / k}^{\bullet}$, so

$$
\omega=\sum_{(k, \mathscr{P})} \overline{e\left(\xi_{k, \mathscr{P}}, k, \mathscr{P}\right)},
$$

we know that $\omega$ is overconvergent iff there exist constants $C_{1}>0, C_{2} \in \mathbb{R}$ such that

$$
|k| \leq C_{1} \operatorname{ord}_{p} \xi_{k, \mathscr{D}}+C_{2} \text { for all }(k, \mathscr{P}) .
$$

We can also express overconvergence on $W \Omega_{A / k}^{\bullet}$ by using the Gauss norms $\left\{\gamma_{\varepsilon}\right\}_{\varepsilon>0}$ obtained as quotient norms of the canonical Gauss norms on $W \Omega_{S / k}^{\bullet}$ that we defined before. An $\omega \in W \Omega_{A / k}$ is overconvergent if there exist $\varepsilon>0, C \in \mathbb{R}$ such that $\gamma_{\varepsilon}(\omega) \geq C$. If we use another presentation $S^{\prime}=k\left[U_{1}, \ldots, U_{r^{\prime}}\right] \rightarrow A$, then the associated set of quotient norms $\left\{\delta_{\varepsilon}\right\}_{\varepsilon>0}$ on $W \Omega_{A / k}$ is equivalent to the set $\left\{\gamma_{\varepsilon}\right\}_{\varepsilon>0}$. Here, the notion of equivalence is defined in the same way as for Witt vectors ([5] Definition 2.12).

Proposition 1.2. - (a) We denote by $f \in A$ an arbitrary element. Let $d \in \mathbb{Z}$ be nonnegative. The presheaf

$$
W^{\dagger} \Omega_{\operatorname{Spec} A / k}^{d}\left(\operatorname{Spec} A_{f}\right):=W^{\dagger} \Omega_{A_{f} / k}^{d}
$$

is a sheaf for the Zariski topology on Spec A (compare [7] 0, 3.2.2).

(b) The Zariski cohomology of these sheaves vanishes in degrees $j>0$, i.e.

$$
H_{\mathrm{Zar}}^{j}\left(\operatorname{Spec} A, W^{\dagger} \Omega_{\mathrm{Spec} A / k}^{d}\right)=0 \text {. }
$$

We fix generators $t_{1}, \ldots, t_{r}$ of $A$ and denote by $\left[t_{1}\right], \ldots,\left[t_{r}\right]$ the Teichmüller representatives in $W(A)$. An elementary Witt differential in the variables $\left[t_{1}\right], \ldots,\left[t_{r}\right]$ is the image of a basic Witt differential in variables $\left[T_{1}\right], \ldots,\left[T_{r}\right]$ under the map $\lambda$.

Before we prove the proposition, we need a special description of an overconvergent element $z$ in $W^{\dagger} \Omega_{A_{f} / k}^{d}$. Let $[f] \in W(A)$ be the Teichmüller representative. Hence $\frac{1}{[f]}=\left[\frac{1}{f}\right]$ is the Teichmüller of $\frac{1}{f}$ in $W\left(A_{f}\right)$. For the element $z$ we have the following description.

Proposition 1.3. - The element $z \in W^{\dagger} \Omega_{A_{f} / k}^{d}$ can be written as a convergent series

$$
z=\sum_{l=0}^{\infty} \frac{1}{[f]^{r_{l}}} \bar{\eta}_{l}
$$

where $\bar{\eta}_{l}$ is a finite sum of elementary Witt differentials $\bar{\eta}_{l}^{(t)}$ in the variables $\left[t_{1}\right], \ldots,\left[t_{r}\right]$, images of basic Witt differentials $\eta_{l}^{(t)}$ in variables $\left[T_{1}\right], \ldots,\left[T_{r}\right]$ with weights $k_{l}^{t}$ satisfying the following growth condition:

$\exists C_{1}>0, C_{2} \in \mathbb{R}$ such that for each summand $\eta_{l}^{(t)}$ we have

$$
r_{l}+\left|k_{l}^{t}\right| \leq C_{1} \operatorname{ord}_{p} \eta_{l}^{(t)}+C_{2}
$$

Furthermore we require that for a given $K>0$,

$$
\min _{t} \operatorname{ord}_{p} \eta_{l}^{(t)}>K \text { for almost all } l \text {. }
$$

Proof. - We use here an extended version of basic Witt differentials to the localized polynomial algebra $k\left[T_{1}, \ldots, T_{r}, Y, Y^{-1}\right]$ (compare [8]). A basic Witt differential $\alpha$ in $W \Omega_{k\left[T_{1}, \ldots, T_{r}, Y, Y^{-1}\right] / k}$ has one of the following shapes: 
I) $\alpha$ is a classical basic Witt differential in variables $\left[T_{1}\right], \ldots,\left[T_{r}\right],[Y]$.

II) Let $e\left(\xi_{k, \mathscr{D}}, k, \mathscr{P}\right)$ be a basic Witt differential in variables $\left[T_{1}\right], \ldots,\left[T_{r}\right]$. Then

II 1) $\alpha=e\left(\xi_{k, \mathscr{D}}, k, \mathscr{P}\right) d \log [Y]$

II 2) $\alpha=[Y]^{-r} e\left(\xi_{k, \mathscr{D}}, k, \mathscr{P}\right)$ for some $r>0, r \in \mathbb{N}$

II 3) $\alpha=F^{s} d[Y]^{-l} e\left(\xi_{k, \mathscr{P}}, k, \mathscr{P}\right)$ for some $l>0, p \nmid l, s \geq 0$.

III) $\alpha=V^{u}\left(\xi[Y]^{p^{u} k_{Y}}[T]^{p^{u} k_{I_{0}}}\right) d^{V^{u\left(I_{1}\right)}}[T]^{p^{u\left(I_{1}\right)} k_{I_{1}}} \ldots F^{-t\left(I_{d}\right)} d[T]^{p^{t\left(I_{d}\right)} k_{I_{d}}}$ (compare [10],

In particular, for each such $\alpha$ we have a weight function $k$ on variables $\left[T_{1}\right], \ldots,\left[T_{r}\right]$ with partition $I_{0} \cup \cdots \cup I_{d}=\mathscr{P}, u>0, k_{Y} \in \mathbb{Z}\left[\frac{1}{p}\right]_{<0}$,

$$
u\left(k_{Y}\right) \leq u=\max \left\{u\left(I_{0}\right), u\left(k_{Y}\right)\right\} \text { (notations as in [10]). }
$$

If $I_{0}=\varnothing$, we require $u=\max \left\{u\left(I_{1}\right), u\left(k_{Y}\right)\right\}$.

IV) $\alpha=d \alpha^{\prime}$ when $\alpha^{\prime}$ is as in III).

It follows from loc.cit. that each $\omega \in W \Omega_{k\left[T_{1}, \ldots, T_{r}, Y, Y^{-1}\right] / k}^{\bullet}$ is in a unique way a convergent sum of basic Witt differentials. Here convergent is meant with respect to the canonical filtration on the de Rham-Witt complex.

It is straightforward to show that $\omega$ is overconvergent iff there exist $\widetilde{C_{1}}>0, \widetilde{C_{2}} \in \mathbb{R}$, such that the basic Witt differentials $\alpha$ appearing in the decomposition of $\omega$ have the following properties.

- If $\alpha$ of type I) or of type II 1) occurs as a summand in $\omega$, we require

$$
|k| \leq \widetilde{C_{1}} \operatorname{ord}_{p} \xi_{k, \mathscr{D}}+\widetilde{C_{2}} .
$$

- If $\alpha$ is of type II 2) or II 3) occurs as a summand in $\omega$ then

$$
r+|k| \leq \widetilde{C_{1}} \operatorname{ord}_{p} \xi_{k, \mathscr{P}}+\widetilde{C_{2}} \text { (with } r=l \cdot p^{s} \text { in case II } 3 \text { ). }
$$

- If $\alpha$ is of type III) or IV), then

$$
\left|k_{Y}\right|+\sum_{j=0}^{d}\left|k_{I_{j}}\right| \leq \widetilde{C_{1}} \operatorname{ord}_{p}\left(V^{u} \xi\right)+\widetilde{C_{2}}
$$

(here, $\left|k_{Y}\right|=-k_{Y},\left|k_{I_{j}}\right|=\sum_{i \in I_{j}} k_{i}$ ).

We have a surjective map of complexes:

$$
W^{\dagger} \Omega_{k\left[T_{1}, \ldots, T_{r}, Y, Y^{-1}\right] / k}^{\bullet} \rightarrow W^{\dagger} \Omega_{A_{f} / k}^{\bullet} .
$$

We may represent the $z$ of the proposition as the image of an overconvergent $\omega$, which is a sum of basic Witt differentials as described above. To obtain the representation of $z$ in the proposition, we expand the images of the basic Witt differentials $\alpha$ separately.

In case of condition III) we consider the first factor $V^{u}\left(\xi[Y]^{p^{u} k_{Y}}[T]^{p^{u} k_{I_{0}}}\right)$. For simplicity we assume $I_{0}=\varnothing$; this does not affect the following calculations. Let $-k_{Y}=\frac{r}{p^{u}}$ and 
$l \leq \frac{r}{p^{u}}<l+1$ for an integer $l$. We have

$$
\begin{aligned}
V^{u}\left(\xi[Y]^{p^{u}} k_{Y}\right) & =V^{u}\left(\xi \frac{1}{[Y]^{r}}\right)=V^{u}\left(\xi \frac{1}{[Y]^{l p^{u}}} \cdot \frac{1}{[Y]^{r-l p^{u}}}\right) \\
& =\frac{1}{[Y]^{l}} V^{u}\left(\xi \frac{1}{[Y]^{r-l p^{u}}}\right)=\frac{1}{[Y]^{l}} V^{u}\left(\xi \frac{[Y]^{p^{u}-r+l p^{u}}}{[Y]^{p^{u}}}\right) \\
& =\frac{1}{[Y]^{l+1}} V^{u}\left(\xi[Y]^{(l+1) p^{u}-r}\right) .
\end{aligned}
$$

Now consider the image of $\alpha$ in $W \Omega_{A_{f} / k}^{d}$ where

$$
[Y] \rightarrow[f], \quad\left[Y^{-1}\right] \rightarrow\left[f^{-1}\right], \quad\left[T_{i}\right] \rightarrow\left[t_{i}\right] .
$$

The factor $\frac{1}{[Y]^{l+1}} V^{u}\left(\xi[Y]^{(l+1) p^{u}-r}\right)$ is mapped to $\frac{1}{[f]^{l+1}} V^{u}\left(\xi[f]^{(l+1) p^{u}-r}\right)$.

Represent $f$ as a polynomial of degree $g$ in $t_{1}, \ldots, t_{r}$. Then it is easy to see that the image of $\alpha$ in $W \Omega_{A_{f} / k}^{d}$ is of the form $\frac{1}{[f]^{l+1}} \widetilde{\bar{\eta}}$ where $\widetilde{\bar{\eta}}$ is a (possibly infinite) sum of images of basic Witt differentials $\hat{\eta}^{t}$ in variables $\left[T_{1}\right], \ldots,\left[T_{r}\right]$ with weights $k^{t}$ satisfying

$$
\begin{aligned}
\left|k^{t}\right| & \leq g\left(l+1-\frac{r}{p^{u}}\right)+\sum_{j=0}^{d}\left|k_{I_{j}}\right| \\
& \leq g+\sum_{j=0}^{d}\left|k_{I_{j}}\right| .
\end{aligned}
$$

The case $d \alpha$ (type IV) is deduced from the case III by applying $d$ to $\alpha$ and the Leibniz rule to the image of $d \alpha$ in $W \Omega_{A_{f} / k}^{d}$. So if the image of $\alpha$ as above is $\frac{1}{[f]^{l+1}} \widetilde{\bar{\eta}}$ then the image of $d \alpha$ is

$$
\begin{aligned}
\frac{1}{[f]^{l+1}} d \widetilde{\bar{\eta}}-\frac{1}{[f]^{l+2}} \cdot l d[f] \widetilde{\bar{\eta}} & =\frac{1}{[f]^{l+2}}([f] d \widetilde{\bar{\eta}}-l d[f] \widetilde{\bar{\eta}}) \\
& =\frac{1}{[f]^{l+2}} \widetilde{\overline{\bar{\eta}}}
\end{aligned}
$$

where $\widetilde{\widetilde{\eta}}$ is a sum of images of basic Witt differentials $\widetilde{\widetilde{\eta}}^{t}$ in variables $\left[T_{1}\right], \ldots,\left[T_{r}\right]$ with weights $k^{t}$ satisfying

$$
\left|k^{t}\right| \leq 2 g+\sum_{j=0}^{d}\left|k_{I_{j}}\right| .
$$

We can also compute the images of $\alpha$ in $W \Omega_{A_{f_{i}} / k}$ where $\alpha$ is of type I or II and obtain again a representation

$$
\frac{1}{[f]^{r}} \widetilde{\bar{\eta}} \text { for } r \geq 0
$$

These cases are easier and omitted.

Now we return to the original element $z \in W^{\dagger} \Omega_{A_{f} / k}^{d}$. We may write $z$ as a convergent sum

$$
z=\sum_{m=0}^{\infty} \widetilde{\omega}_{m}
$$

where $\widetilde{\omega}_{m}$ is an elementary Witt differential being the image of a basic Witt differential $\alpha_{m}$ in $W \Omega_{k\left[T_{1}, \ldots, T_{r}, Y, Y^{-1}\right] / k}$ of type I, II, III or IV. 
In all cases we have a representation

$$
\widetilde{\omega}_{m}=\frac{1}{[f]^{r_{m}}} \widetilde{\bar{\eta}}_{m}
$$

where $\widetilde{\bar{\eta}}_{m}$ is the sum of images of basic Witt differentials $\tilde{\eta}_{m}^{t}$ in variables $\left[T_{1}\right], \ldots,\left[T_{r}\right]$ with weights $k_{m}^{t}$ such that

$$
r_{m}+\left|k_{m}^{t}\right| \leq \widetilde{C_{1}} \operatorname{ord}_{p}\left(\widetilde{\eta}_{m}^{t}\right)+\widetilde{C_{2}}+2(g+1)
$$

Now consider - for a given integer $N$ - the element $z$ modulo $\mathrm{Fil}^{N}$, so the image $\bar{z}^{(N)}$ of $z$ in

$$
W_{N} \Omega_{A_{f} / k}^{d}=W_{N} \Omega_{A / k}^{d} \bigotimes_{W_{N}(A)} W_{N}(A)\left[\frac{1}{[f]}\right] .
$$

One then finds a lifting $z^{(N)}$ of $\bar{z}^{(N)}$ in $W \Omega_{A_{f} / k}$ such that $z^{(N)}=\sum_{m=0}^{b(N)} \omega_{m}$ is a finite sum, i.e.

$$
\omega_{m}=\frac{1}{[f]^{r_{m}}} \bar{\eta}_{m}
$$

where now $\bar{\eta}_{m}$ is a finite sum of images of basic Witt differentials $\eta_{m}^{t}$ in variables $\left[T_{1}\right], \ldots,\left[T_{r}\right]$ satisfying the growth condition

$$
r_{m}+\left|k_{m}^{t}\right| \leq C_{1} \operatorname{ord}_{p}\left(\eta_{m}^{t}\right)+C_{2}
$$

with $C_{1}:=\widetilde{C_{1}}, C_{2}=\widetilde{C_{2}}+2(g+1)$.

The elements $z^{(N)}$ can be chosen to be compatible for varying $N$ and we have $z=\lim z^{(N)}$. It is clear that the second condition of the lemma is also satisfied, this finishes the proof of Proposition 1.3.

Remark. - It will later be convenient to express the assertion in Proposition 1.3 using Gauss norms. Let $\left\{\gamma_{\varepsilon}\right\}_{\varepsilon>0}$ be the set of Gauss norms on $W \Omega_{A / k}$ obtained as quotient norms from the canonical Gauss norms on $W \Omega_{S / k}$ using the presentation $S \rightarrow A$. Let $\left\{\delta_{\varepsilon}\right\}_{\varepsilon>0}$ be the set of Gauss norms on $W \Omega_{A_{f} / k}$ obtained as quotient norms using the presentation $\widetilde{S}:=k\left[T_{1}, \ldots, T_{r}, U\right] \rightarrow A_{f}, T_{i} \mapsto t_{i}, U \mapsto \frac{1}{f}$. We now define another set of Gauss norms as follows. For $\omega \in W \Omega_{A_{f} / k}$ we consider the collection of all possible representations

$$
\omega=\sum_{l \geq 0}[f]^{-l} \eta_{l}, \text { for } \eta_{l} \in W \Omega_{A / k}
$$

such that for a given $t$, almost all $\eta_{l}$ are zero in $W_{t+1} \Omega_{A / k}$. We set

$$
\gamma_{\varepsilon}^{\text {quot }}(\omega)=\sup \left\{\inf _{l}\left\{\gamma_{\varepsilon}\left(\eta_{l}\right)-l \varepsilon\right\}\right\}
$$

where the sup is taken over all possible representations (*). Then Proposition 1.3 is equivalent to the assertion that the set $\left\{\gamma_{\varepsilon}^{\text {quot }}\right\}_{\varepsilon>0}$ is equivalent to the set $\left\{\delta_{\varepsilon}\right\}_{\varepsilon>0}$. Equally, we will obtain an equivalent set of Gauss norms $\left\{\gamma_{\varepsilon}^{\prime}\right\}_{\varepsilon>0}$ if in the above definition we only allow representations such that the exponents of $f$ are all divisible by $p$. 
Now we are ready to prove Proposition 1.2.

As $W \Omega^{\bullet}$ is a complex of Zariski sheaves we need to show - in order to prove part (a) of the proposition - the following claim:

Let $z \in W \Omega_{A / k}^{d}$ for some fixed $d$, let $\left\{f_{i}\right\}_{i}$ be a collection of finitely many elements in $A$ that generate $A$ as an ideal. Assume that for each $i$ the image $z_{i}$ of $z$ in $W \Omega_{A_{f_{i}} / k}^{d}$ is already in $W^{\dagger} \Omega_{A_{f_{i} / k}}^{d}$. Then $z \in W^{\dagger} \Omega_{A / k}^{d}$.

Let $\left[f_{i}\right]$ be the Teichmüller of $f_{i}$ with inverse $\frac{1}{\left[f_{i}\right]}=\left[\frac{1}{f_{i}}\right]$.

Lemma 1.4. - There are elements $r_{i} \in W^{\dagger}(A)$ such that $\sum_{i=1}^{n} r_{i}\left[f_{i}\right]=1$.

Proof. - Consider a relation $\sum_{i=1}^{n} a_{i} f_{i}=1$ in $A$. Then $\sum_{i=1}^{n}\left[a_{i}\right]\left[f_{i}\right]=1+{ }^{V} \eta \in W^{\dagger}(A)$. By Lemma 2.25 in [5],

Define $r_{i}=\left(1+{ }^{V} \eta\right)^{-1} \cdot\left[a_{i}\right]$.

$$
\left(1+{ }^{V} \eta\right)^{-1} \in W^{\dagger}(A) .
$$

Lemma 1.5. - For each there are polynomials $Q_{i, t}\left[T_{1}, \ldots, T_{2 n}\right]$ in $2 n$ variables such that

(1) degree $Q_{i, t} \leq 3 \cdot n t$

(2) $\sum_{i=1}^{n} Q_{i, t}\left(\left[f_{1}\right], \ldots,\left[f_{n}\right], r_{1}, \ldots, r_{n}\right)\left[f_{i}\right]^{t}=1$.

For the proof of this lemma, compare [13].

We know that $\operatorname{Spec} A=\cup_{i=1}^{n} D\left(f_{i}\right)$. For a tuple $1 \leq i_{1}<\cdots<i_{m} \leq n$, let $\mathfrak{U}_{i_{1} \ldots i_{m}}=\cap_{j=1}^{m} D\left(f_{i_{j}}\right)$. Fix $d \in \mathbb{N}$ and let

$$
\begin{aligned}
C^{m} & =C^{m}\left(\operatorname{Spec} A, W^{\dagger} \Omega_{A / k}^{d}\right) \\
& =\oplus_{1 \leq i_{1}<\cdots<i_{m} \leq n} W^{\dagger} \Omega_{A_{f_{i_{1}} \cdots f_{i_{m}}} / k}^{d} \\
& =\oplus_{1 \leq i_{1}<\cdots<i_{m} \leq n} \Gamma\left(\mathfrak{U}_{i_{1} \ldots i_{m}}, W^{\dagger} \Omega_{A / k}^{d}\right) .
\end{aligned}
$$

Then consider the Čech complex

$$
0 \rightarrow C^{0} \rightarrow C^{1} \rightarrow C^{2} \rightarrow \cdots
$$

We have $C^{0}=W^{\dagger} \Omega_{A / k}^{d}$ and $C^{0} \rightarrow C^{1}$ is the restriction map $W^{\dagger} \Omega_{A / k}^{d} \rightarrow W^{\dagger} \Omega_{A_{f_{i}} / k}^{d}$ for all $i$. It is then clear that Proposition 1.2 follows from the following.

Proposition 1.6. - The complex $C^{\bullet}$ is exact.

Proof. - The proof is very similar to the proof of Lemma 7 in [13]. We fix as before $k$-algebra generators $t_{1}, \ldots, t_{r}$ of $A$. Suppose $\sigma \in C^{m}, m \geq 2$, is a cocycle. Then $\sigma$ has components

$$
\sigma_{i_{1} \ldots i_{m}} \in \Gamma\left(\mathfrak{U}_{i_{1} \ldots i_{m}}, W^{\dagger} \Omega_{\operatorname{Spec} A / k}^{d}\right)=W^{\dagger} \Omega_{A_{f_{i_{1}} \ldots f_{i_{m}}} / k}^{d} .
$$

Applying Proposition 1.3 we see that $\sigma_{i_{1} \ldots i_{m}}$ has a representation as an overconvergent sum of Witt differentials as follows: $\sigma_{i_{1} \ldots i_{m}}=\sum_{l=0}^{\infty} M_{l}^{i_{1} \ldots i_{m}}$ with

$$
M_{l}^{i_{1} \ldots i_{m}}=\sum_{j} \frac{1}{\left[f_{i_{1} \ldots i_{m}}\right]^{j}} \bar{\eta}_{l i_{1} \ldots i_{m}}^{(j)} \text { a finite sum }
$$


where $\left[f_{i_{1} \ldots i_{m}}\right]^{j}:=\left[f_{i_{1}}\right]^{j} \cdots\left[f_{i_{m}}\right]^{j}, \bar{\eta}_{l i_{1} \ldots i_{m}}^{(j)}$ is a sum of images of basic Witt differentials $\eta_{d i_{1} \ldots i_{m}}^{\left(j_{t}\right)}$ in variables $\left[T_{1}\right], \ldots,\left[T_{r}\right],\left(T_{i} \rightarrow t_{i}\right)$ and weights $k_{l i_{1} \ldots i_{m}}^{\left(j_{t}\right)}$ satisfying

i) $j+\left|k_{l i_{1} \ldots i_{m}}^{\left(j_{t}\right)}\right| \leq C\left(\operatorname{ord}_{p} \eta_{l i_{1} \ldots i_{m}}^{\left(j_{t}\right)}+1\right)$

ii) $l \geq \operatorname{ord}_{p} \eta_{l i_{1} \ldots i_{m}}^{\left(j_{j}\right)} \geq l-1$.

Notation: We say that $M_{l}^{i_{1} \ldots i_{r}}$ has degree $\leq C(l+1)$.

We shall construct a cochain $\tau$ so that $\partial \tau=\sigma$. The reduced complex

$$
C^{\bullet} / \mathrm{Fil}^{n} C^{\bullet}=C^{\bullet}\left(\left\{D\left(f_{i}\right)\right\}_{i}, W_{n} \Omega_{A / k}^{\bullet}\right)
$$

is exact. We will inductively construct a sequence of cochains

$$
\tau_{k}=\sum_{1 \leq i_{1}<\cdots<i_{m-1} \leq n} \tau_{k i_{1} \ldots i_{m-1}}
$$

such that the sum

$$
\sum_{k=0}^{\infty} \tau_{k}
$$

converges in $C^{m-1}$ to a coboundary of $\sigma$. The $\tau_{k}$ are chosen to satisfy the following properties:

(1) $\partial\left(\sum_{k=0}^{l-1} \tau_{k}\right)=\sigma$ modulo $\mathrm{Fil}^{2^{l}-1} C^{m}$

(2) $\tau_{0 i_{1} \ldots i_{m-1}} \in W^{\dagger} \Omega_{A_{f_{i_{1}} \cdots f_{i_{m}}} / k}$, and $\tau_{k i_{1} \ldots i_{m-1}} \in \mathrm{Fil}^{2^{k}-1} W^{\dagger} \Omega_{A_{f_{i_{1}} \cdots f_{i_{m}}} / k}$ for $k \geq 1$.

(3) $\tau_{k i_{1} \ldots i_{m-1}} \in W \Omega_{A / k}^{\mathrm{fin}}\left[\left[f_{1}\right], \ldots,\left[f_{n}\right], r_{1}, \ldots, r_{n}, \frac{1}{\left[f_{i_{1} \ldots i_{m-1}}\right]}\right]$ to be understood as a polynomial in the "variables" $\left[f_{1}\right], \ldots,\left[f_{n}\right], r_{1}, \ldots, r_{n}$ and $\frac{1}{\left[f_{\left.i_{1} \ldots i_{m-1}\right]}\right]}$ with the coefficients being finite sums of elementary Witt differentials in $\left[t_{1}\right], \ldots,\left[t_{r}\right]$ such that the total degree (with $\left[t_{1}\right], \ldots,\left[t_{r}\right]$ contributing to the degree via possibly fractional weights) is bounded by $24 n C 2^{k}$. We write degree $\tau_{k i_{1} \ldots i_{m-1}} \leq 24 n C 2^{k}$.

(4) $\left[f_{i_{\alpha}}\right]^{C 2^{k+1}} \tau_{k i_{1} \ldots i_{m-1}} \in W \Omega_{A / k}^{\mathrm{fin}}\left[\left[f_{1}\right], \ldots,\left[f_{n}\right], r_{1}, \ldots, r_{n}, \frac{1}{\left[f_{i_{1} \ldots \hat{i}_{\alpha} \ldots i_{m-1}}\right]}\right]$ with degree $\left[f_{i_{\alpha}}\right]^{C 2^{k+1}} \tau_{k i_{1} \ldots i_{m-1}} \leq C 2^{k+1}+24 n C 2^{k}$.

Then (2) implies that all the coefficients $\eta$ of the polynomial representation (3) satisfy $\operatorname{ord}_{p} \eta \geq 2^{k}-1$. Also (1) implies that $\partial\left(\sum_{k=0}^{\infty} \tau_{k}\right)=\sigma$. Using (2) and (3) we will show that $\sum_{k=0}^{\infty} \tau_{k} \in C^{m-1}$, i.e. is overconvergent.

Define elements $\sigma_{s i_{1}, \ldots, i_{m}} \in W \Omega_{A_{f_{i_{1}} \ldots f_{i_{m}}}}^{d}$ for $n \geq 0$ by

$$
\sigma_{s i_{1}, \ldots, i_{m}}=\sum_{\alpha=0}^{2^{s+1}-1} M_{\alpha}^{i_{1} \ldots i_{m}} .
$$

Then $\sigma_{s i_{1}, \ldots, i_{m}} \equiv \sigma_{i_{1}, \ldots, i_{m}} \bmod \mathrm{Fil}^{2^{s+1}}$ and degree $\sigma_{s i_{1}, \ldots, i_{m}} \leq C 2^{2+1}$.

Define the cochain $\tau_{0} \in C^{m-1}$ by

$$
\tau_{0 i_{1} \ldots i_{m-1}}=\sum_{i=1}^{n} \alpha_{i, 2 C}\left[f_{i}\right]^{2 C} \sigma_{0 i_{1}, \ldots, i_{m} i} .
$$


Suppose we have constructed, for some integer $s>0$, cochains $\tau_{k} \in C^{m-1}$ for $0 \leq k<s$ satisfying (1)-(4). Then we construct $\tau_{s}$ as follows: Let $\gamma_{s i_{1} \ldots i_{m}}=\sigma_{s i_{1}, \ldots, i_{m}}-\partial\left(\sum_{k=0}^{s-1} \tau_{k}\right)_{i_{1} \ldots i_{m}}$. We see that $\gamma_{s i_{1} \ldots i_{m}} \in \mathrm{Fil}^{2^{s}-1} C^{m}$ is a cocycle modulo $\mathrm{Fil}^{2^{s}+1} C^{m}$ and degree $\gamma_{s i_{1} \ldots i_{m}} \leq 24 n C 2^{s-1}$.

Define

$$
\tau_{s i_{1} \ldots i_{m-1}}=\sum_{i=1}^{n} Q_{i, C 2^{s+1}}\left[f_{i}\right]^{C 2^{s+1}} \gamma_{s i_{1} \ldots i_{m-1} i} .
$$

Then $\sum_{k=0}^{s} \tau_{k}$ satisfies (1) by ([EGA], III.1.2.4.). We have

$$
\begin{aligned}
{\left[f_{i}\right]^{C 2^{s+1}} \gamma_{s i_{1} \ldots i_{m-1} i} } & \in W^{\dagger} \Omega_{A_{f_{i_{1}} \cdots f_{i_{m-1}}}} \cap \mathrm{Fil}^{2^{s}-1} W^{\dagger} \Omega_{f_{i_{1}} \cdots f_{i_{m}} / k} \\
& =\mathrm{Fil}^{2^{s}-1} W^{\dagger} \Omega_{A_{f_{i_{1}} \cdots f_{i_{m-1}}}}
\end{aligned}
$$

and therefore $\tau_{s i_{1} \ldots i_{m-1}}$ satisfies (2) (we have used (4) for $\tau_{k}, k<s$ ). Moreover, $\tau_{s i_{1} \ldots i_{m-1}}$ has total degree bounded by

$$
24 n C 2^{s-1}+3 n C 2^{s+1}+C 2^{s+1} \leq 24 n C 2^{s}
$$

and $\tau_{s}$ satisfies (3). It is straightforward to show property (4) for $\tau_{s}$. Therefore it remains to show that $\sum_{k=0}^{\infty} \tau_{k}$ is overconvergent. This will be derived from properties (2) and (3) as follows.

It follows from (3) that $\tau_{s i_{1} \ldots i_{m-1}}$ can be written as a finite sum $\tau_{s i_{1} \ldots i_{m-1}}=\sum_{I} r^{I} M_{s, I}$, where $I$ runs through a finite set of multi-indices in $\mathbb{N}_{0}^{n}, r^{I}=r_{1}^{\lambda_{1}} \cdots r_{n}^{\lambda_{n}}$ for $I=\left(\lambda_{1}, \ldots, \lambda_{n}\right)$ and $M_{s, I}$ is a finite sum of images of basic Witt differentials $\omega_{s}^{t}$ in variables $\left[T_{1}\right], \ldots,\left[T_{r}\right]$, $\left[Y_{1}\right], \ldots,\left[Y_{n}\right],[Z]$ with

$$
\left[T_{j}\right] \mapsto\left[t_{j}\right],\left[Y_{j}\right] \mapsto\left[f_{j}\right],[Z] \mapsto \prod_{j=1}^{m-1} \frac{1}{\left[f_{i_{j}}\right]}
$$

with weights $k_{s}^{t}$ satisfying

$$
|I|+\left|k_{s}^{t}\right| \leq 24 n C 2^{s}=C^{\prime} 2^{s}
$$

$\left(C^{\prime}:=24 n C\right)$ and

$$
\begin{aligned}
\operatorname{ord}_{p} \omega_{s}^{t} \geq 2^{s}-1 & =\frac{1}{C^{\prime}}\left(C^{\prime} 2^{s}\right)-1 \\
& \geq \frac{1}{C^{\prime}}\left(|I|+\left|k_{s}^{t}\right|\right)-1
\end{aligned}
$$

For fixed $I$ and varying $s$ we get a sum

$$
\sum_{s} r^{I} M_{s, I}=r^{I} \sum_{s} M_{s, I}
$$

Because of the condition $(*), \omega_{I}=\sum_{s} M_{s, I}$ is overconvergent with radius of convergence $\varepsilon=\frac{1}{C^{\prime}}$ and

$$
\hat{\gamma}_{\frac{1}{C^{\prime}}}\left(\omega_{I}\right) \geq \frac{1}{C^{\prime}}|I|-1
$$

Here $\hat{\gamma}_{\varepsilon}$ is the quotient norm of the canonical $\gamma_{\varepsilon}$ on $W \Omega_{k\left[T_{1}, \ldots, T_{r}, Y_{1}, \ldots, Y_{n}, Z\right] / k}$.

We now look again at the definition of $r_{i}$. There exist liftings $\widetilde{\eta}, \widetilde{r}_{i}$ of $\eta, r_{i}$ in $W^{\dagger}(S)$ and $\widetilde{a}_{i}$ of $a_{i}$ in $S$ where $\widetilde{\eta}$ is a finite sum of homogeneous elements such that

$$
\widetilde{r}_{i}=\left(1+{ }^{V} \widetilde{\eta}\right)^{-1}\left[\widetilde{a}_{i}\right] .
$$


For $\delta:=\frac{1}{C^{\prime}}$, there exist $\varepsilon>0, \frac{1}{C^{\prime}}>\varepsilon$ such that

$$
\breve{\gamma}_{\varepsilon}\left({ }^{V} \widetilde{\eta}\right) \geq-\delta
$$

because we have a finite sum of homogeneous elements. By [5] Lemma 2.25,

$$
\breve{\gamma}_{\varepsilon}\left(\widetilde{r}_{i}\right) \geq-\delta \text { as well. }
$$

Let $\widetilde{\omega}_{I}$ be a lifting of $\omega_{I}$ in $W^{\dagger} \Omega_{k\left[T_{1}, \ldots, T_{r}, Y_{1}, \ldots, Y_{n}, Z\right] / k}$ such that $\hat{\gamma}_{\varepsilon}\left(\omega_{I}\right)=\gamma_{\varepsilon}\left(\widetilde{\omega}_{I}\right)$. Then we obtain by Corollary 0.16 ,

$$
\begin{aligned}
\hat{\gamma}_{\varepsilon}\left(r^{I} \omega_{I}\right) & \geq \gamma_{\varepsilon}\left(\widetilde{r}^{I} \widetilde{\omega}_{I}\right) \\
& \geq \gamma_{\varepsilon}\left(\widetilde{\omega}_{I}\right)+\breve{\gamma}_{\varepsilon}\left(\widetilde{r}^{I}\right) \\
& =\hat{\gamma}_{\varepsilon}\left(\omega_{I}\right)+\breve{\gamma}_{\varepsilon}\left(\widetilde{r}^{I}\right) \\
& \geq \hat{\gamma}_{\frac{1}{C^{\prime}}}\left(\omega_{I}\right)+\breve{\gamma}_{\varepsilon}\left(\widetilde{r}^{I}\right) \\
& \geq \delta|I|-1+|I|(-\delta)=-1 .
\end{aligned}
$$

As this holds for all $I$, we see that $\sum_{s=0}^{\infty} \tau_{s i_{1} \ldots i_{m-1}}$ is overconvergent with radius of convergence $\varepsilon$, and hence Proposition 1.6 follows, and so does Proposition 1.2.

REMARK. - The above final arguments in the proof of Proposition 1.2 are very similar to the proof that $W^{\dagger}(A)$ is weakly complete in the sense of Monsky-Washnitzer (compare [14] and Proposition 2.28 of [5]). Hence $W^{\dagger} \Omega_{A / k}^{d}$ satisfies a certain property of weak completeness in positive degrees as well.

COROLlary 1.7. - The complex $W^{\dagger} \Omega_{\mathrm{Spec} A / k}$, defined for each affine scheme as above, extends to a complex of Zariski sheaves $W^{\dagger} \Omega_{X / k}$ on any variety $X / k$.

In the remainder of this section and the next, we prove the following.

Theorem 1.8. - Let $X$ be a smooth variety. Then $W^{\dagger} \Omega_{X / k}^{\bullet}$ defines a complex of étale sheaves on $X$.

Proof. - As $W^{\dagger} \Omega_{X / k}^{\bullet}$ is a complex of Zariski sheaves on $X$, the problem of being a sheaf on the étale site is local on $X$. By a result of Kedlaya [9] any smooth variety $X$ has a covering by affine smooth schemes Spec $A$ which are finite étale over distinguished opens in an affine space $\mathbb{A}_{k}^{n}$. It therefore suffices to show that if $A$ is a finite étale extension over a localized polynomial algebra, $A^{\prime}$ a standard étale extension of $A$, then an element $z$ in $W \Omega_{A / k}^{d}$ that becomes overconvergent in $W \Omega_{A^{\prime} / k}^{d}$ is already overconvergent over $A$. By localizing further we may assume first that there is an element $f$ in $A$ such that $A_{f}^{\prime}$ is finite étale over $A_{f}$, of the form $A_{f}^{\prime}=A_{f}[X] /(p(X))$ for some monic irreducible polynomial $p(X)$. The following proposition reduces the argument to the case $A_{f}=A_{f}^{\prime}$; hence we will need to show

$$
W \Omega_{A / k}^{d} \cap W^{\dagger} \Omega_{A_{f} / k}^{d}=W^{\dagger} \Omega_{A / k}^{d} .
$$


Proposition 1.9. - Let $B$ be a finite étale and monogenic A-algebra, where $A$ is smooth over a perfect field of char $p>0$. Let $B=A[X] /(f(X))$ for a monic irreducible polynomial $f(X)$ of degree $m=[B: A]$ such that $f^{\prime}(X)$ is invertible in $B$. Let $[x]$ be the Teichmüller of the element $X \bmod f(X)$ in $W(B)$. Then we have for each $d \geq 0$ a direct sum decomposition of $W^{\dagger}(A)$-modules

$$
W^{\dagger} \Omega_{B / k}^{d}=W^{\dagger} \Omega_{A / k}^{d} \oplus W^{\dagger} \Omega_{A / k}^{d}[x] \oplus \cdots \oplus W^{\dagger} \Omega_{A / k}^{d}[x]^{m-1} .
$$

Proof. - From Corollary 2.46 in [5] we know that this proposition is true for $d=0$ :

$W^{\dagger}(B)$ is a finite $W^{\dagger}(A)$-module with basis $1,[x], \ldots,[x]^{m-1}$. There is a unique lifting $\widetilde{f}(X) \in W^{\dagger}(A)[X]$ of $f(X)$ such that $W^{\dagger}(B)=W^{\dagger}(A)[X] / \widetilde{f}(X)$ and $\tilde{f}^{\prime}([x])$ is invertible in $W^{\dagger}(B)$. In particular $W^{\dagger}(B)$ is étale over $W^{\dagger}(A)$.

Let $\tilde{f}(X)=X^{m}+a_{m-1} X^{m-1}+\cdots+a_{1} X+a_{0}$, with $a_{i} \in W^{\dagger}(A)$ and

$$
\frac{1}{\widetilde{f}^{\prime}([x])}=c_{m-1}[x]^{m-1}+\cdots+c_{1}[x]+c_{0},
$$

with $c_{i} \in W^{\dagger}(A)$.

When we consider an element $z$ in $W^{\dagger} \Omega_{B / k}^{d}$ with radius of convergence $\varepsilon>0$ we will always assume that $\varepsilon$ is small enough such that all $a_{j}, c_{j}, j=0, \ldots, m-1$ are in $W^{\varepsilon}(A)$.

The equation

$$
\widetilde{f}([x])=[x]^{m}+a_{m-1}[x]^{m-1}+\cdots+a_{1}[x]+a_{0}=0
$$

(note that $\tilde{f}(X)$ is the minimal polynomial of $[x]$ over $W^{\dagger}(A)$ ) implies that

$$
d \tilde{f}([x])=0 .
$$

Hence we get

$$
\tilde{f}^{\prime}([x]) d[x]+d a_{m-1}[x]^{m-1}+\cdots+d a_{1}[x]+d a_{0}=0 .
$$

As $\left(\tilde{f}^{\prime}([x])\right)^{-1}$ has coefficients in $W^{\varepsilon}(A)$ and $W^{\varepsilon}(A)$ is a ring we see that

$$
\begin{aligned}
d[x] & =-\frac{1}{\widetilde{f^{\prime}([x])}}\left(d a_{m-1}[x]^{m-1}+\cdots+d a_{1}[x]+d a_{0}\right) \\
& =\sum_{l, j=0}^{m-1} \lambda_{l_{j}} d a_{l}[x]^{j} \text { with } \lambda_{l}, a_{l} \in W^{\varepsilon}(A) .
\end{aligned}
$$

The elements $a_{l} \in W^{\varepsilon}(A)$ are homogeneous as they are elementary symmetric functions in the $\left[t_{i}\right]$, where $\left[t_{i}\right], i=1, \ldots, m$ are the roots of $\tilde{f}$, lifting the roots $t_{i}$ of $f$.

We have $\lambda_{l_{j}} d a_{l}=d\left(a_{l} \lambda_{l_{j}}\right)-a_{l} d \lambda_{l_{j}}$ by the Leibniz rule. The elements $a_{l} \lambda_{l_{j}}$ are in $W^{\varepsilon}(A)$, hence $d\left(a_{l} \lambda_{l_{j}}\right) \in W^{\varepsilon} \Omega_{A / k}^{1}$. As $a_{l}$ is homogeneous, the element $a_{l} d \lambda_{l_{j}}$ is in $W^{\varepsilon} \Omega_{A / k}^{1}$ as well (Corollary 0.16 ). So we get

$$
d[x] \in W^{\varepsilon} \Omega_{A / k}^{1} \oplus \cdots \oplus W^{\varepsilon} \Omega_{A / k}^{1}[x]^{m-1} .
$$


One proves similarly that

$$
d[x]^{i} \in W^{\varepsilon} \Omega_{A / k}^{1} \oplus \cdots \oplus W^{\varepsilon} \Omega_{A / k}^{1}[x]^{m-1}
$$

for all $i, 1 \leq i \leq m-1$.

Let $b_{1}, \ldots, b_{r}$ be generators of the $k$-algebra $A$ and $z \in W^{\dagger} \Omega_{B / k}^{d}$ be an overconvergent sum of elementary Witt differentials $z_{i}$ in variables $\left[b_{1}\right], \ldots,\left[b_{r}\right],[x]$ with $\gamma_{\varepsilon}\left(z_{i}\right)>C$ for all $i$. If in $z_{i}$ the variable $[x]$ occurs with integral weight $k_{x}$ we may assume $1 \leq k_{x} \leq m-1$. If $[x]$ belongs to the interval $I_{0}$ with underlying partition $\mathscr{P}$ corresponding to $z_{i}$, then evidently $z_{i}=\eta_{i}[x]^{k_{x}}$ with $\eta_{i}$ an elementary Witt differential in the variables $\left[b_{1}\right], \ldots,\left[b_{r}\right]$ with $\gamma_{\varepsilon}\left(\eta_{i}\right)>C$. If $[x]$ occurs with integral weight $k_{x}, 1 \leq k_{x} \leq m-1$, and belongs to the interval $I_{j}, j \geq l$, then after applying the Leibniz rule and the previous case we see that

$$
z_{i}=\omega_{i}+\eta_{i} d[x]^{k_{x}}
$$

with $\omega_{i} \in W^{\varepsilon} \Omega_{A / k}^{d} \oplus \cdots \oplus W^{\varepsilon} \Omega_{A / k}^{d}[x]^{m-1}$ and $\eta_{i} \in W^{\varepsilon} \Omega_{A / k}^{d-1}$ with $\gamma_{\varepsilon}\left(\eta_{i}\right)>C$. In addition, all coefficients $\omega_{i}^{(j)}$ in $W^{\varepsilon} \Omega_{A / k}^{d}$ satisfy $\gamma_{\varepsilon}\left(\omega_{i}^{(j)}\right)>C$. We may also assume that all coefficients $\beta_{i}^{(j)}$ of $d[x]^{i}$ in $W^{\varepsilon} \Omega_{A / k}^{1}$ for all $1 \leq i \leq m-1$ satisfy $\gamma_{\varepsilon}\left(\beta_{i}^{(j)}\right)>C$. Then,

$$
\eta_{i} d[x]^{k_{x}} \in W^{\frac{\varepsilon}{2}} \Omega_{A / k}^{d} \oplus \cdots \oplus W^{\frac{\varepsilon}{2}} \Omega_{A / k}^{d}[x]^{m-1}
$$

and we have for all coefficients $\delta_{i}^{(j)} \in W^{\frac{\varepsilon}{2}} \Omega_{A / k}^{d}$ that occur in this representation of $\eta_{i} d[x]^{k_{x}}$ that

$$
\gamma_{\frac{\varepsilon}{2}}\left(\delta_{i}^{(j)}\right)>C
$$

Now we use [5] Corollary 2.46. If $\alpha=\sum_{i=0}^{m-1} \xi_{i} x^{i} \in W^{\varepsilon}(B)$ satisfies $\gamma_{\varepsilon}(\alpha)>C$ then $\xi_{i} \in W^{\varepsilon}(A)$ with $\gamma_{\varepsilon}\left(\xi_{i}\right)>C^{\prime}$ and $C^{\prime}$ only depends on $C$ and $\varepsilon$; wlog $C^{\prime}<C$.

Assume that in an elementary Witt differential $z_{i}$ occurring in the overconvergent $z$ we have

$$
z_{i}={ }^{t} \eta \cdot d \omega
$$

and $[x]$ occurs in $\eta$ with fractional weight $k_{x}, k_{x}=\frac{i}{p^{t}}, 1 \leq i \leq m-1$. Then applying the above fact we see that

$$
z_{i} \in W^{\varepsilon} \Omega_{A / k}^{d} \oplus \cdots \oplus W^{\varepsilon} \Omega_{A / k}^{d}[x]^{m-1}
$$

and the coefficients $z_{i}^{(j)}$ satisfy $\gamma_{\varepsilon}\left(z_{i}^{(j)}\right)>C^{\prime}$.

If $[x]$ occurs with fractional weight $k_{x}$ in an interval $I_{j}, j \geq 1$, of the underlying partition of $z_{i}$, then by combining the previous cases we see that

$$
z_{i} \in W^{\frac{\varepsilon}{2}} \Omega_{A / k}^{d} \oplus \cdots \oplus W^{\frac{\varepsilon}{2}} \Omega_{A / k}^{d}[x]^{m-1}
$$

and all coefficients $z_{i}^{(j)}$ satisfy $\gamma_{\frac{\varepsilon}{2}}\left(z_{i}^{(j)}\right)>C^{\prime}$.

This implies that the original $z \in W^{\varepsilon} \Omega_{B / k}^{d}$ with $\gamma_{\varepsilon}(z)>C$ has a representation

$$
z=\sum_{i=0}^{m-1} \sigma_{i}[x]^{i} \in W^{\frac{\varepsilon}{2}} \Omega_{A / k}^{d} \oplus \cdots \oplus W^{\frac{\varepsilon}{2}} \Omega_{A / k}^{d}[x]^{m-1}
$$

with $\gamma_{\frac{\varepsilon}{2}}\left(\sigma_{i}\right)>C^{\prime}$ for all $i=0, \ldots, m-1$. 
On the other hand, by possibly applying the Leibniz rule repeatedly, it is clear that an element in

$$
W^{\dagger} \Omega_{A / k}^{d} \oplus \cdots \oplus W^{\dagger} \Omega_{A / k}^{d}[x]^{m-1}
$$

can be represented as an overconvergent sum of elementary Witt differentials in variables $\left[b_{1}\right], \ldots,\left[b_{r}\right],[x]$, and hence lies in $W^{\dagger} \Omega_{B / k}^{d}$. This finishes the proof of the proposition.

REMARK. - Note that the isomorphism in the proposition is a restriction of the isomorphism

$$
W \Omega_{B / k}^{d} \cong W(B) \bigotimes_{W(A)} W \Omega_{A / k}^{d} \cong \bigoplus_{i=0}^{m-1} W \Omega_{A / k}^{d}[x]^{i}
$$

for the completed de Rham-Witt complex. As $W(B)$ is finite étale over $W(A)$ if B is finite étale over $A$, this latter isomorphism is a consequence of étale base change for the de Rham-Witt complex of finite level, by passing to the inverse limit (compare [10] Proposition 1.7 and Corollary 2.46 in [5]).

To prove the theorem, it remains to show that

$$
W \Omega_{B / k}^{d} \cap W^{\dagger} \Omega_{B g / k}^{d}=W^{\dagger} \Omega_{B / k}^{d}
$$

for a $k$-algebra $B$ which is a finite étale extension over a localization $A_{f}$ of a polynomial algebra $A=k\left[T_{1}, \ldots, T_{d}\right]$, and some $g \in B$. After possibly localizing again, we may assume wlog that $g$ itself is in the polynomial algebra. After applying Proposition 1.9 again, we reduce the proof of the étale sheaf property to the case where $B=A_{f}$. That is, we need to prove (1.10) in the special case $B=A_{f}$ and $g \in A$. This will follow from a further careful study of the Gauss norm properties on the de Rham-Witt complex of the polynomial algebra $A$ and a localization $A_{f}$, done in the next section.

\section{Gauss norm properties on the de Rham-Witt complex of localized polynomial algebras}

We will consider the Gauss norms on the truncated de Rham-Witt complexes $W_{t+1} \Omega_{A / k}$ and $W_{t+1} \Omega_{A_{f} / k}$ (and also $W_{t+1} \Omega_{A_{f g} / k}$ ) and describe overconvergence on the completed de Rham-Witt complexes via these truncated Gauss norms. Before we can do this, we need to review a few more properties of the de Rham complex $\Omega_{A / k}$ for the polynomial algebra $A=k\left[T_{1}, \ldots, T_{d}\right]$ over a perfect field $k$ of characteristic $p>0$.

We recall the basic differentials $\mathfrak{e}(k, \mathscr{P})$ from $(0.1)$ :

$$
\mathfrak{e}(k, \mathscr{P})=T^{k_{I_{0}}}\left(\frac{d T^{k_{I_{1}}}}{p^{\operatorname{ord}_{p} k_{I_{1}}}}\right) \cdots\left(\frac{d T^{k_{I_{l}}}}{p^{\operatorname{ord}_{p} k_{I_{l}}}}\right) .
$$

A basic differential is called primitive if $I_{0} \neq \varnothing$ and if the function $k$ is not divisible by $p$.

Proposition 2.2. - Let $\mathfrak{e}(k, \mathscr{P})$ be a primitive basic differential. Then for all $1 \leq j \leq d$

$$
T_{j}^{p} \mathfrak{e}(k, \mathscr{P})
$$

is a linear combination of primitive basic differentials with coefficients in $k$. 
Proof. - Let $I_{0}=\left\{i_{1}, \ldots, i_{t}\right\}$. Let $I_{0}^{\prime}=\left\{i_{1}, \ldots, i_{s}\right\} \subset I_{0}$ be the subset of all indices $i_{m}$, such that $\operatorname{ord}_{p} k_{i_{m}}=0$. Let $I_{0}^{\prime \prime}$ be the complement of $I_{0}^{\prime}$ in $I_{0}$. We have $I_{0}^{\prime} \neq \varnothing$ but possibly $I_{0}^{\prime \prime}=\varnothing$.

Consider the case where $j=i_{m} \in I_{0}^{\prime}$. We define $k^{\prime}$ such that $k_{i_{m}}^{\prime}=k_{i_{m}}+p$ and $k_{j}^{\prime}=k_{j}$ for all other indices. Then $\operatorname{Supp} k=\operatorname{Supp} k^{\prime}$ and the chosen order on these sets is the same. From this we see that

$$
T_{j}^{p} \mathfrak{e}(k, \mathscr{P})=\mathfrak{e}\left(k^{\prime}, \mathscr{P}\right) .
$$

Now we consider the case where $j$ does not belong to $I_{0}^{\prime}$. We write

$$
T_{j}^{p} T^{k_{I_{0}^{\prime \prime}}}\left(\frac{d T^{k_{I_{1}}}}{p^{\operatorname{ord}_{p} k_{I_{1}}}}\right) \cdots\left(\frac{d T^{k_{I_{l}}}}{p^{\operatorname{ord}_{p} k_{I_{l}}}}\right)
$$

as a linear combination of basic differentials $\mathfrak{e}(h, Q)$ for possibly different partitions $Q$. Let $\iota$ be the weight such that $\iota(j)=p$ and such that $\iota$ vanishes on the remaining indices. Then $h=k+\iota$.

Consider the subcase where $\operatorname{ord}_{p} k_{j}=0$. Then $j$ must belong to one of the sets $I_{0}, \ldots, I_{r}$ and therefore $j$ must be bigger than any of the indices appearing in $I_{0}^{\prime}$. Then

$$
T^{k_{I_{0}^{\prime}}^{\prime}(h, Q)}
$$

is a primitive basic differential for each partition $Q$. Its weight function $k^{\prime \prime}$ is the sum of $k_{\mid I_{0}^{\prime}}$ (the restriction of $k$ to $I_{0}^{\prime}$ ) and $h$. That we obtain a basic differential follows from the fact that for the order given by $k^{\prime \prime}$ any element of $I_{0}^{\prime}$ precedes any element in Supp $h$.

This last sentence is still true in the subcase $\operatorname{ord}_{p} k_{j}>0$, because this implies $\operatorname{ord}_{p} h_{j}>0$. This finishes the proof.

We consider $\Omega_{A / k}$ throughout this section as an $A$-module via restriction of scalars by $F: A \rightarrow A$. We will say that we consider $\Omega_{A / k}$ as an $A-F$-module.

Proposition 2.3. - Let $P^{l} \subset \Omega_{A / k}^{l}$ be the $k$-subvector space generated by primitive basic differentials. We have a direct decomposition:

$$
\Omega_{A / k}^{l}=P^{l} \oplus d P^{l-1} \oplus F \Omega_{A / k}^{l} .
$$

Each summand on the right hand side is a free A-F-module which has a basis consisting of basic differentials

Proof. - The decomposition (2.4) is direct because the second $k$-vector space is generated by basic differentials whose weights are not divisible by $p$ and such that we have $I_{0}=\varnothing$ in the partition while $F \Omega_{A / k}^{l}$ is generated by basic differentials whose weights are divisible by $p$.

It follows from Proposition 2.2 that $P^{l}$ is an $A-F$-module. Then the other two summands of (2.4) are clearly $A-F$-modules. Therefore all summands are projective $A$ - $F$-modules. All summands are graded by the absolute value of weights and are therefore graded $A-F$-modules. Let $\mathfrak{a}$ be the ideal of $A$ generated by $T_{1}, \ldots, T_{d}$. A basis of the $A-F$-module $P^{l}$ is obtained by lifting a basis of the (graded) $k$-vector space $P^{l} / F \mathfrak{a} P^{l}$. This proves the last sentence of the proposition.

4 e SÉRIE - TOME $44-2011-$ No $^{\circ} 2$ 
Next we consider the de Rham-Witt complex $W \Omega_{A / k}$. We denote by $\mathrm{Fil}^{n}$ the kernel of the canonical map $W \Omega_{A / k} \rightarrow W_{n} \Omega_{A / k}$. It is an abelian group generated by the basic Witt differentials $e(\xi, k, \mathscr{P})$ such that $\operatorname{ord}_{V} \xi \geq n$ (compare [10]). We set:

$$
G^{n, l}=\mathrm{Fil}^{n} W \Omega_{A / k}^{l} / \mathrm{Fil}^{n+1} W \Omega_{A / k}^{l} .
$$

We consider it as a $W(A)-F$-module. Clearly the module structure factors via $W(A) \rightarrow A$. We consider throughout this $A$-module structure on $G^{n}$. On $G^{0}=\Omega_{A / k}^{l}$ it agrees with the $A-F$-module structure considered above.

The $A$-module $G^{n, l}$ has a direct decomposition into free $A$-modules:

$$
\begin{aligned}
G^{n, l}= & V^{n} P^{l} \oplus p V^{n-1} P^{l} \oplus \cdots \oplus p^{n} P^{l} \\
& \oplus d V^{n} P^{l-1} \oplus p d V^{n-1} P^{l-1} \oplus \cdots \oplus p^{n} d P^{l} \\
& \oplus p^{n} F \Omega_{A / k}^{l} .
\end{aligned}
$$

This follows from Proposition 2.3 and the decompositon of $W \Omega_{A / k}$ defined by basic Witt differentials. It is clear that each summand has a basis consisting of basic Witt differentials.

Proposition 2.6. - For each $n \geq 0$ there is a family $\omega_{i}^{(n)} \in \mathrm{Fil}^{n} W \Omega_{A / k}^{l}$ of basic Witt differentials, where i runs through some finite index set $J_{n}$, satisfying the following:

For each $n$ the elements $\omega_{i}^{(n)}$ for $i \in J_{n}$ form a basis of the A-module $G^{n, l}$.

$A$ Witt differential $\omega \in W_{t+1} \Omega_{A / k}^{l}$ has a unique expression

$$
\omega=\sum_{n=0}^{t} \sum_{i \in J_{n}} F_{[}\left[a_{i}^{(n)}\right] \omega_{i}^{(n)},
$$

where $a_{i}^{(n)} \in A$.

Moreover the truncated Gauss norm $\gamma_{\varepsilon}[t]$ is given by the following formula:

$$
\gamma_{\varepsilon}[t](\omega)=\min _{n, i \in J_{n}}\left\{p \gamma_{\varepsilon}\left(a_{i}^{(n)}\right)+\gamma_{\varepsilon}\left(\omega_{i}^{(n)}\right)\right\} .
$$

Proof. - For a fixed $n$ and each of the summands of (2.5) we choose basic Witt differentials in $\mathrm{Fil}^{n}$ which form a basis of this summand as an $A$-module. Therefore we obtain a basis $\omega_{i}^{(n)}$. Then we write:

$$
\omega=\sum_{i \in J_{0}} F a_{i}^{(0)} \omega_{i}^{(0)} \quad \text { modulo Fil }{ }^{1}
$$

Then we consider the Witt differential

$$
\omega(1)=\omega-\sum_{i \in J_{0}} F\left[a_{i}^{(0)}\right] \omega_{i}^{(0)} \in \mathrm{Fil}^{1} .
$$

Then we consider $\omega(1) \in G^{1, l}$ and express it by the chosen basis of this $A$-module. This process may be continued to obtain the expression (2.7).

Finally we have to prove the assertion about the Gauss norm. We consider first the case of a differential $\omega \in G^{n, l} \subset W_{n+1} \Omega_{A / k}$. We decompose $\omega$ according to the decomposition (2.5):

$$
\omega=\sum \omega_{m}
$$


Since the decomposition (2.5) is defined by a partition of the set of basic Witt differentials we deduce the formula:

$$
\gamma_{\varepsilon}[n]\left(\sum \omega_{m}\right)=\min _{m}\left\{\gamma_{\varepsilon}\left(\omega_{m}\right)\right\}
$$

Let us denote by $S$ an arbitrary summand of the decomposition (2.5). All nonzero elements $\sigma \in S$ have the same order $\operatorname{ord}_{V} \sigma=\mathfrak{o}_{S}$. As explained, $S$ is a free graded module over $A$ :

$$
S=\bigoplus S_{t}
$$

such that $S_{t}$ has a basis of basic Witt differentials whose weights have absolute value $t$. We find that for $z \in S_{t}$, such that $z \neq 0$ :

$$
\gamma_{\varepsilon}[n](z)=\mathfrak{o}_{S}-\varepsilon t
$$

Now we assume that $z=\sum^{F} a_{i}^{(n)} \omega_{i}^{(n)}$. Since $S$ is free we deduce from this the formula:

$$
\gamma_{\varepsilon}[n]\left(\sum{ }^{F} a_{i}^{(n)} \omega_{i}^{(n)}\right)=\min \left\{\gamma_{\varepsilon}\left({ }^{F} a_{i}^{n}\right)+\gamma_{\varepsilon}\left(\omega_{i}^{(n)}\right)\right\}
$$

Now we consider the element $\omega \in W_{t+1} \Omega_{A / k}$ with the expansion (2.7). We set $\gamma_{\varepsilon}[t](\omega)=C$. Then we have:

$$
C \leq \gamma_{\varepsilon}[0](\omega)=\gamma_{\varepsilon}[0]\left(\sum^{F}\left[a_{i}^{(0)}\right] \omega_{i}^{(0)}\right)=\min _{i}\left\{\gamma_{\varepsilon}\left({ }^{F}\left[a_{i}^{(0)}\right]\right)+\gamma_{\varepsilon}\left(\omega_{i}^{(0)}\right)\right\}
$$

On the other hand we have the inequality:

$$
\gamma_{\varepsilon}[t]\left(\sum^{F}\left[a_{i}^{(0)}\right] \omega_{i}^{(0)}\right) \geq \min _{i}\left\{\gamma_{\varepsilon}\left({ }^{F}\left[a_{i}^{(0)}\right]\right)+\gamma_{\varepsilon}\left(\omega_{i}^{(0)}\right)\right\}
$$

We obtain that

$$
\gamma_{\varepsilon}[t]\left(\omega-\sum F^{F}\left[a_{i}^{(0)}\right] \omega_{i}^{(0)}\right) \geq \gamma_{\varepsilon}[t](\omega)=C .
$$

Applying the same argument to $\omega(1)=\omega-\sum^{F}\left[a_{i}^{(0)}\right] \omega_{i}^{(0)} \in \mathrm{Fil}^{1}$ we find that in the decomposition (2.7) the following inequality holds:

$$
\gamma_{\varepsilon}\left({ }^{F}\left[a_{i}^{(n)}\right]\right)+\gamma_{\varepsilon}\left(\omega_{i}^{(n)}\right) \geq C .
$$

But on the other hand we have:

$$
C=\gamma_{\varepsilon}\left(\sum_{n=0}^{t} \sum_{i \in J_{n}} F^{F}\left[a_{i}^{(n)}\right] \omega_{i}^{(n)}\right) \geq \min _{n . i \in J_{n}}\left\{\gamma_{\varepsilon}\left({ }^{F}\left[a_{i}^{(n)}\right]\right)+\gamma_{\varepsilon}\left(\omega_{i}^{(n)}\right)\right\} .
$$

This proves the last assertion.

ReMARK. - Let $f=\sum \alpha_{k} T^{k} \in A$, where $\alpha_{k} \in k$, be a polynomial. We set $\tilde{f}=\sum\left[a_{k}\right][T]^{k} \in W(A)$. This is an integral Witt vector which lifts $f$. We can replace in the proof the Teichmüller representatives $\left[a_{i}^{(n)}\right]$ by $\tilde{a}_{i}^{(n)}$ and the element ${ }^{F}\left[a_{i}^{(n)}\right]$ by the element ${ }^{F} \tilde{a}_{i}^{(n)}$. Then we obtain a unique expression:

$$
\omega=\sum_{n=0}^{t} \sum_{i \in J_{n}}{ }^{F} \tilde{a}_{i}^{(n)} \omega_{i}^{(n)}
$$

The Gauss norm is given by the Formula (2.8).

4 e SÉRIE - TOME $44-2011-$ No $^{\circ} 2$ 
Our next aim is to prove a similar proposition for the localization $A_{f}$ of the polynomial algebra $A=k\left[T_{1}, \ldots, T_{d}\right]$ for an element $f \in A$. We write $\delta=\operatorname{deg} f$.

Let $\omega \in W_{t+1} \Omega_{A_{f} / k}$. We have seen that an admissible pseudovaluation $\gamma_{\varepsilon}^{\prime}[t]$ on this de Rham-Witt complex is obtained as follows. We consider all possible representations:

$$
\omega=\sum_{l}\left(\eta_{l} /[f]^{l p}\right), \quad \text { where } \eta_{l} \in W_{t+1} \Omega_{A / k} .
$$

Then $\gamma_{\varepsilon}^{\prime}[t](\omega)$ is the maximum over all possible numbers

$$
\min \left\{\gamma_{\varepsilon}[t] \eta_{l}-\varepsilon l p\right\}
$$

There is always a representation where this maximum is taken. Such representations will be called optimal. The following inequalities are immediate:

$$
\begin{aligned}
& \gamma_{\varepsilon}^{\prime}[t](\omega) \leq \gamma_{\varepsilon}^{\prime}[t-1](\omega) \\
& \gamma_{\varepsilon}^{\prime}[t](\omega) \leq \gamma_{\delta}^{\prime}[t](\omega) \quad \text { for } \varepsilon \geq \delta .
\end{aligned}
$$

We could also consider all representations of the form $\omega=\sum_{l}\left(\eta_{l} /[f]^{l}\right)$ without the extra factor $p$. Then we denote by $\hat{\gamma}_{\varepsilon}^{\prime}(\omega)$ the maximum of the numbers $\min \left\{\gamma_{\varepsilon}[t] \eta_{l}-\varepsilon l\right\}$. We will use this Gauss norm only for the Witt ring.

We write $\mathrm{Fil}_{f}^{m}=\mathrm{Fil}^{m} W \Omega_{A_{f} / k}$. By étale base change $\mathrm{Fil}_{f}^{m}$ is obtained from $\mathrm{Fil}^{m}$ by localizing with respect to $[f]$.

Lemma 2.11. - Let $\omega \in \mathrm{Fil}_{f}^{m}$. Then there is an optimal representation (2.10) of $\omega$ such that $\eta_{l} \in \mathrm{Fil}^{m}$.

Proof. - The case $m=0$ is trivial. We assume by induction that there is an optimal representation such that $\eta_{l} \in \mathrm{Fil}^{m-1}$. Consider the residue classes of $\bar{\eta}_{l}$ of $\eta_{l}$ in $G^{m-1}=\oplus_{l} G^{m-1, l}=\mathrm{Fil}^{m-1} / \mathrm{Fil}^{m}$. We use the abbreviation $\delta_{\varepsilon}\left(\bar{\eta}_{l}\right)=\gamma_{\varepsilon}[i-1](\eta)$. Clearly we have that $\delta_{\varepsilon}\left(\bar{\eta}_{l}\right) \geq \gamma_{\varepsilon}[t]\left(\eta_{l}\right)$. Then we have in $G^{m-1}$ the relation:

$$
\sum_{l=0}^{M}\left(\bar{\eta}_{l} /[f]^{l}\right)=0 .
$$

We may assume that $\bar{\eta}_{M} \neq 0$ and that $M$ is the minimal possible value for all optimal representations. Then we have to show that $M \geq 1$ is impossible. We see that $\bar{\eta}_{M}$ is divisible by $[f]$. Then we write:

$$
\bar{\eta}_{M}=[f] \bar{\tau} .
$$

We obtain that $\delta_{\varepsilon}(\bar{\tau})-\varepsilon \delta=\delta_{\varepsilon}\left(\bar{\eta}_{M}\right)$. We may lift $\bar{\tau}$ to an element $\tau \in$ Fil $^{m-1}$ such that $\gamma_{\varepsilon}[t](\tau)=\delta_{\varepsilon}(\bar{\tau})$. We write:

$$
\eta_{M}=[f] \tau+\rho, \quad \text { where } \rho \in \mathrm{Fil}^{m} .
$$

Since $\gamma_{\varepsilon}[t]([f] \tau)=\gamma_{\varepsilon}(\tau)-\varepsilon \delta=\delta_{\varepsilon}(\bar{\tau})-\varepsilon \delta=\delta_{\varepsilon}\left(\eta_{m}^{-}\right) \geq \gamma_{\varepsilon}\left(\eta_{M}\right)$ we conclude that $\gamma_{\varepsilon}(\rho) \geq \gamma_{\varepsilon}\left(\eta_{M}\right)$. Now we consider the equation:

$$
\left(\eta_{M} /[f]^{M}\right)=\left(\tau /[f]^{M-1}\right)+\left(\rho /[f]^{M}\right)
$$

Inserting this in (2.10) we obtain again an optimal expression, since:

$$
\begin{aligned}
\gamma_{\varepsilon}(\tau)-(M-1) \varepsilon & \geq \gamma_{\varepsilon}\left(\eta_{M}\right)-M \varepsilon \\
\gamma_{\varepsilon}(\rho)-M \varepsilon & \geq \gamma_{\varepsilon}\left(\eta_{M}\right)-M \varepsilon
\end{aligned}
$$


Reducing this modulo $\mathrm{Fil}^{m}$ we see that the number $M$ becomes smaller.

Lemma 2.13. - Let $\omega \in G^{t} \subset W_{t+1} \Omega_{A_{f} / k}$. Then $\omega$ has a unique expression:

$$
\omega=\sum{ }^{F}\left[c_{i}\right] \omega_{i}^{(t)}, \quad c_{i} \in A_{f} .
$$

Then we have:

$$
\gamma_{\varepsilon}^{\prime}[t](\omega)=\min _{i \in J_{n}}\left\{p \hat{\gamma}_{\varepsilon}^{\prime}\left(c_{i}\right)+\gamma_{\varepsilon}\left(\omega_{i}^{(t)}\right)\right\} .
$$

Proof. - Since $G^{t}$ is a free $A-F$-module it is clear that the localization is a free $A_{f}-F$-module with the same basis. From this it follows that such a decomposition exists.

We choose an optimal representation:

$$
\omega=\sum_{l}\left(\eta_{l} /[f]^{p l}\right)
$$

By the last lemma we may assume that $\eta_{l} \in G^{t}$. Then we find for $\eta_{l}$ an expression:

$$
\eta_{l}=\sum{ }^{F} a_{i l} \omega_{i}^{(t)}, \quad a_{i l} \in A .
$$

Therefore we obtain by definition and Proposition 2.6:

$$
\gamma_{\varepsilon}^{\prime}[t](\omega)=\min _{i l}\left\{\gamma_{\varepsilon}[t]\left(\sum{ }^{F} a_{i l} \omega_{i}^{(t)}\right)-\varepsilon l p\right\}=\min _{i l}\left\{p \gamma_{\varepsilon}\left(a_{i l}\right)+\gamma_{\varepsilon}\left(\omega_{i}^{(t)}\right)-\varepsilon l p\right\}
$$

We set

$$
c_{i}^{(n)}=\sum_{l}\left(a_{i l}^{(n)} / f^{l}\right)
$$

We can assume that this expression is optimal for $\hat{\gamma}_{\varepsilon}^{\prime}$. Because in the other case we could insert the optimal expression in the equation:

$$
\omega=\sum_{i}\left(\sum_{l}{ }^{F} a_{i l} / f^{l p}\right) \omega_{i}^{(t)} .
$$

This would make the right hand side of (2.15) bigger. But then (2.16) would again be an optimal expression of the form (2.14).

We obtain $\hat{\gamma}_{\varepsilon}^{\prime}\left(c_{i}^{(n)}\right)=\min _{l}\left\{\gamma_{\varepsilon}\left(a_{i l}^{(n)}\right)-\varepsilon l\right\}$. This shows the last formula of the lemma.

Let $c \in A_{f}$ be an element. We choose an optimal representation:

$$
c=\sum\left(a_{l} / f^{l}\right) .
$$

We set:

$$
\hat{c}=\sum\left[a_{l}\right] /[f]^{l} \in W_{t+1}\left(A_{f}\right) .
$$

We find

$$
\hat{\gamma}_{\varepsilon}^{\prime}[t](\hat{c}) \geq \hat{\gamma}_{\varepsilon}^{\prime}(c)
$$

But the other inequality is obvious since $\hat{\gamma}_{\varepsilon}^{\prime}[t](\hat{c}) \leq \hat{\gamma}_{\varepsilon}^{\prime}[1](\hat{c})$. Therefore we have an equation:

$$
\hat{\gamma}_{\varepsilon}^{\prime}[t](\hat{c})=\hat{\gamma}_{\varepsilon}^{\prime}(c) \text {. }
$$

In the same way we obtain:

$$
\gamma_{\varepsilon}^{\prime}[t](\hat{c})=\gamma_{\varepsilon}^{\prime}(c)
$$

Indeed we have:

$$
\gamma_{\varepsilon}^{\prime}\left({ }^{F} c\right)=p \hat{\gamma}_{\varepsilon}^{\prime}(c) \quad \text { for } c \in A_{f}
$$

$4{ }^{\text {e }}$ SÉRIE - TOME $44-2011-$ N $^{\circ} 2$ 
To see this we can reduce to the case, where $f$ is regular with respect to one variable. Then one uses that reduced representations are optimal.

Proposition 2.20. - With the same notation as in Proposition 2.6 consider a Witt differential $\eta \in W_{t+1} \Omega_{A_{f} / k}$. Then there is a unique decomposition:

$$
\eta=\sum_{i, n}{ }^{F} \hat{c}_{i}^{(n)} \omega_{i}^{(n)}, \quad c_{i}^{(n)} \in A_{f} .
$$

The truncated Gauss norm is given by the formula:

$$
\gamma_{\varepsilon}^{\prime}[t](\eta)=\min _{i, n}\left\{p \hat{\gamma}_{\varepsilon}^{\prime}\left(c_{i}^{(n)}\right)+\gamma_{\varepsilon}\left(\omega_{i}^{(n)}\right)\right\} .
$$

Proof. - Since $t$ is fixed we will set $\gamma_{\varepsilon}^{\prime}=\gamma_{\varepsilon}^{\prime}[t]$ Consider an expression in $\mathrm{Fil}^{m}$ :

$$
z=\sum_{i}{ }^{F} \hat{c}_{i}^{(m)} \omega_{i}^{(m)}
$$

We claim that:

$$
\gamma_{\varepsilon}^{\prime}(z)=\gamma_{\varepsilon}^{\prime}[m](z)=\min _{i}\left\{\gamma_{\varepsilon}^{\prime}\left({ }^{F} \hat{c}_{i}^{(m)}\right)+\gamma_{\varepsilon}\left(\omega_{i}^{(m)}\right)\right\} .
$$

Indeed, the second equality follows from Lemma 2.13. We see easily that $\gamma_{\varepsilon}^{\prime}(z)$ is greater than the right hand side of (2.21). Indeed, we choose optimal representations for $c_{i}^{(m)}$ :

$$
c_{i}^{(m)}=\sum_{l} a_{i l} / f^{l}
$$

We obtain:

$$
z=\sum_{l, i}\left[a_{i l}\right]^{p} \omega_{i}^{(m)} /[f]^{l p}=\sum_{l}\left(\sum_{i}\left[a_{i l}\right]^{p} \omega_{i}^{(m)}\right) /[f]^{l p} .
$$

This shows that

$$
\begin{aligned}
\gamma_{\varepsilon}^{\prime}(z) & \geq \min _{l}\left\{\gamma_{\varepsilon}\left(\sum_{i}\left[a_{i l}\right]^{p} \omega_{i}^{(m)}\right)-l p \varepsilon\right\} \\
& =\min _{l}\left\{\min _{i}\left\{p \gamma_{\varepsilon}\left(a_{i l}\right)+\gamma_{\varepsilon}\left(\omega_{i}^{(m)}\right)\right\}-l p \varepsilon\right\} .
\end{aligned}
$$

The last equation follows from Proposition 2.6. By definition we have the equation:

$$
p \hat{\gamma}_{\varepsilon}\left(c_{i}^{(m)}\right)=\min \left\{\gamma_{\varepsilon}\left(a_{i l}\right)-l \varepsilon\right\} .
$$

This shows the inequality:

$$
\gamma_{\varepsilon}^{\prime}(z) \geq \min \left\{\gamma_{\varepsilon}^{\prime}\left({ }^{F} \hat{c}_{i}^{(m)}\right)+\gamma_{\varepsilon}\left(\omega_{i}^{(m)}\right)\right\} .=\gamma_{\varepsilon}^{\prime}[m](z) .
$$

On the other hand we have $\gamma_{\varepsilon}^{\prime}(z) \leq \gamma_{\varepsilon}^{\prime}[m](z)$, and this proves the equality (2.21).

As in the proof of Proposition 2.6 we find an expansion with the desired properties.

Remark. - Consider the natural map $B=k\left[T_{1}, \ldots, T_{d}, S\right] \rightarrow A_{f}$, which maps $S$ to $f^{-1}$. We have defined the overconvergent Witt vectors $W^{\dagger} \Omega_{A_{f} / k}$ as the image of $W^{\dagger} \Omega_{B / k}$ by the canonical map:

$$
W \Omega_{B / k} \rightarrow W \Omega_{A_{f} / k} .
$$

Assume that we are given $\omega \in W \Omega_{A_{f} / k}$, such that there is a constant $C$ with

$$
\gamma_{\varepsilon}^{\prime}[t](\omega) \geq C
$$


for all $t \geq 0$. We claim that $\omega \in W^{\dagger} \Omega_{A_{f} / k}$. By the unicity statement of the last proposition we have an infinite expansion:

$$
\omega=\sum_{i, n} F_{\hat{c}_{i}^{(n)}} \omega_{i}^{(n)}
$$

As in the proof above we take optimal representations:

$$
c_{i}^{(n)}=\sum_{l} a_{i l}^{(n)} / f^{l}
$$

Then we find a convergent sum in the Fil-topology:

$$
\omega=\sum_{l}\left(\sum_{i}\left[a_{i l}^{n}\right]^{p} \omega_{i}^{(n)}\right) /[f]^{l p},
$$

where $p \gamma_{\varepsilon}\left(a_{i l}^{n}\right)-\varepsilon l p+\gamma_{\varepsilon}\left(\omega_{i}^{(n)}\right) \geq C$. But then

$$
\sum_{l}\left(\sum_{i}\left[a_{i l}^{n}\right]^{p} \omega_{i}^{(n)}\right)[S]^{l p} \in W \Omega_{B / k}
$$

is clearly an overconvergent Witt differential which lifts $\omega$. Conversely the condition (2.23) is clearly fulfilled for an overconvergent $\omega$, because $\gamma_{\varepsilon}^{\prime}$ is equivalent to the quotient norm induced by (2.22).

Corollary 2.24. - For $\eta \in W_{t+1} \Omega_{A_{f} / k}$ we have the equation:

$$
\gamma_{\varepsilon}^{\prime}[t+1](p \eta)=1+\gamma_{\varepsilon}^{\prime}[t](\eta)
$$

Proof. - We note that the proposition holds for each set $\omega_{i}^{(n)} \in W \Omega_{A / k}$ of basic Witt differentials which for each given $n$ induce a basis of $G^{n}$ as $A-F$-module. But clearly $p \omega_{i}^{(n)}$ is part of a basis of $G^{n+1}$ consisting of basic Witt differentials. This gives with the notations of the proposition:

$$
\gamma_{\varepsilon}^{\prime}[t+1](\eta)=\gamma_{\varepsilon}^{\prime}\left(\sum_{i, n} F_{\hat{c}_{i}}^{(n)}\left(p \omega_{i}^{(n)}\right)\right)=\min \left\{p \hat{\gamma}_{\varepsilon}\left(c_{i}^{(n)}\right)+\gamma_{\varepsilon}\left(p \omega_{i}^{(n)}\right)\right\} .
$$

This proves the result.

Proposition 2.25. - Let $f, g \in A$ be two non-zero elements without common divisors. There is a constant $Q>1$ with the following property. Let t be a rational number and let $\varepsilon>0$ be a real number. We denote by $\gamma_{\varepsilon}^{\prime}=\gamma_{\varepsilon}^{\prime}[t]$ the natural Gauss norm on $W_{t+1} \Omega_{A_{f} / k}$ and by $\gamma_{\varepsilon}^{\prime \prime}$ the natural Gauss norm on $W_{t+1} \Omega_{A_{f g} / k}$.

We denote the image of a Witt differential $\omega \in W_{t+1} \Omega_{A_{f} / k}$ in $W_{t+1} \Omega_{A_{f g} / k}$ by the same letter. Then the following inequality holds:

$$
\begin{aligned}
& \gamma_{\varepsilon / Q}^{\prime}(\omega) \geq \gamma_{\varepsilon}^{\prime \prime}(\omega) \\
& \gamma_{\varepsilon / Q}^{\prime \prime}(\omega) \geq \gamma_{\varepsilon}^{\prime}(\omega) .
\end{aligned}
$$


Proof. - We begin with the proof of the first inequality, which is the nontrivial one. We may extend the ground field $k$ and assume that $k$ is infinite. After a coordinate change we may assume that $f$ and $g$ are regular with respect to $T_{1}$. Consider an element $c \in A_{f}$ with the reduced representation

$$
c=\sum a_{l} / f^{l}
$$

If we regard $c$ as an element of $A_{f g}$ it has the reduced representation:

$$
c=\sum\left(a_{l} g^{l}\right) /(f g)^{l} .
$$

We have defined a lifting $\hat{c} \in W\left(A_{f}\right)$ of $c$ (2.17). This coincides with the lifting $\hat{c} \in W\left(A_{f g}\right)$ :

$$
\sum\left[a_{l}\right] /[f]^{l}=\sum\left(\left[a_{l} g^{l}\right]\right) /([f g])^{l} .
$$

We set $C=\gamma_{\varepsilon}^{\prime \prime}(\omega)$. By Proposition 2.20 we have the expansion:

$$
\omega=\sum_{i, n} \hat{c}_{i}^{(n)} \omega_{i}^{(n)}, \quad c_{i}^{n} \in A_{f} .
$$

Since the $\hat{c}$ with respect to $A_{f}$ and with respect to $A_{f g}$ means the same (2.27) is also the expansion of $\omega$ with respect to $A_{f g}$ according to Proposition 2.20.

Therefore we conclude that:

$$
C=\min \left\{p \hat{\gamma}_{\varepsilon}^{\prime \prime}\left(c_{i}^{(n)}\right)+\gamma_{\varepsilon}\left(\omega_{i}^{(n)}\right)\right\}
$$

By Proposition 1.30 of [5] there are constants which depend only on $\operatorname{deg} f$ and $\operatorname{deg} g$, such that the pseudovaluation $\hat{\gamma}_{\varepsilon}^{\prime}$ on $A_{f}$ (respectively $\hat{\gamma}_{\varepsilon}^{\prime \prime}$ on $A_{f g}$ ) compare to the $\mu$-functions:

$$
\begin{aligned}
Q_{1} \mu^{\prime}(c) & \leq \hat{\gamma}_{\varepsilon}^{\prime}(c) \leq Q_{2} \mu^{\prime}(c) \text { for } c \in A_{f}, \\
Q_{1} \mu^{\prime \prime}(d) & \leq \hat{\gamma}_{\varepsilon}^{\prime \prime}(d) \leq Q_{2} \mu^{\prime \prime}(d) \text { for } d \in A_{f g} .
\end{aligned}
$$

If $c \in A_{f}$ has denominator $f^{n}$, then $c$ regarded as an element of $A_{f g}$ has denominator $(f g)^{n}$. This shows the equality

$$
\mu^{\prime}(c)=\mu^{\prime \prime}(c)
$$

We find the inequalities:

$$
\hat{\gamma}_{\varepsilon}^{\prime \prime}(c) \leq Q_{2} \mu^{\prime \prime}(c)=Q_{2} \mu^{\prime}(c) \leq\left(Q_{2} / Q_{1}\right) \hat{\gamma}_{\varepsilon}^{\prime}(c) .
$$

We set $Q=\max \left\{1,\left(Q_{1} / Q_{2}\right)\right\}$ and rewrite the above inequality:

$$
\hat{\gamma}_{\varepsilon}^{\prime \prime}(c) \leq \hat{\gamma}_{\varepsilon / Q}^{\prime}(c), \quad \text { for } c \in A_{f}
$$

From this we find:

$$
p \hat{\gamma}_{\varepsilon / Q}^{\prime}\left(c_{i}^{(n)}\right)+\gamma_{\varepsilon / Q}\left(\omega_{i}^{(n)}\right) \geq p \hat{\gamma}_{\varepsilon}^{\prime \prime}\left(c_{i}^{(n)}\right)+\gamma_{\varepsilon}\left(\omega_{i}^{(n)}\right) \geq C .
$$

Using Proposition 2.20 this implies the first inequality (2.26).

The second inequality is straightforward: We choose an optimal representation of $\omega \in W_{t+1} \Omega_{A_{f} / k}$ with respect to $\varepsilon$

$$
\omega=\eta_{l} /[f]^{l p}, \quad \eta_{l} \in W_{t+1} \Omega_{A / k} .
$$

From the representation

$$
\omega=\eta_{l} g^{l p} /[f g]^{l p}, \quad \eta_{l} \in W_{t+1} \Omega_{A / k}
$$


we obtain that:

$$
\begin{aligned}
\gamma_{\varepsilon / Q}^{\prime \prime}(\omega) & \geq \gamma_{\varepsilon / Q}\left(\eta_{l}[g]^{l p}\right)-l p \varepsilon / Q \\
& =\gamma_{\varepsilon / Q}\left(\eta_{l}\right)-l p \varepsilon(\operatorname{deg} g+1) / Q \\
& \geq \gamma_{\varepsilon}\left(\eta_{l}\right)-\varepsilon l p=\gamma_{\varepsilon}^{\prime}(\omega) .
\end{aligned}
$$

Using the remark before Corollary 2.24, we see that Proposition 2.25 implies the claim in (1.10) and finishes the proof of Theorem 1.8.

COROLlary 2.28. - With the notations of the proposition we have the inequality:

$$
\gamma_{\varepsilon}^{\prime}\left([g]^{p} \omega\right) \leq \gamma_{\varepsilon / Q^{2}}^{\prime}(\omega)+p \varepsilon / Q .
$$

Let $c \in A_{f}$, such that $c \neq 0$. Then there are constants $C, Q \in \mathbb{R}, Q>1$, such that for every $\omega \in W_{t+1} \Omega_{A_{f} / k}$

$$
\gamma_{\varepsilon}^{\prime}([c] \omega) \leq \gamma_{\varepsilon / Q^{2}}^{\prime}(\omega)+C \varepsilon
$$

This shows in particular that an element $\omega \in W \Omega_{A_{f} / k}$ is overconvergent if for some $c \in A_{f}, c \neq 0$, the element $[c] \omega$ is overconvergent.

Proof. - We begin to show the inequalities:

$$
\begin{aligned}
& \gamma_{\varepsilon}^{\prime}\left(\frac{1}{\left[f^{p}\right]} \omega\right) \geq \gamma_{\varepsilon}^{\prime}(\omega)-p \varepsilon \\
& \gamma_{\varepsilon}^{\prime}\left(\left[f^{p}\right] \omega\right) \leq \gamma_{\varepsilon}^{\prime}(\omega)+p \varepsilon .
\end{aligned}
$$

To verify the first of these inequalities we choose an optimal representation:

$$
\omega=\sum_{l} \eta_{l} /[f]^{l p}
$$

After dividing by $[f]^{p}$ we conclude:

$$
\gamma_{\varepsilon}^{\prime}\left(\frac{1}{\left[f^{p}\right]} \omega\right) \geq \min _{l}\left\{\gamma_{\varepsilon}\left(\eta_{l}\right)-(l+1) p \varepsilon\right\}=\gamma_{\varepsilon}^{\prime}(\omega)-p \varepsilon .
$$

From this we deduce formally the second inequality:

$$
\gamma_{\varepsilon}^{\prime}(\omega)=\gamma_{\varepsilon}^{\prime}\left(\frac{1}{\left[f^{p}\right]}[f]^{p} \omega\right) \geq \gamma_{\varepsilon}^{\prime}\left([f]^{p} \omega\right)-p \varepsilon
$$

Let $h \in A$ be arbitrary. If we multiply (2.31) by [h] we obtain the inequality

$$
\gamma_{\varepsilon}^{\prime}([h] \omega) \geq \gamma_{\varepsilon}(h)+\gamma_{\varepsilon}^{\prime}(\omega) .
$$

As above we obtain from this formally:

$$
\gamma_{\varepsilon}^{\prime}\left(\frac{1}{\left[f^{p}\right]} \omega\right) \leq \gamma_{\varepsilon}^{\prime}(\omega)-\gamma_{\varepsilon}\left([f]^{p}\right) .
$$

Using (2.30) for $\gamma_{\varepsilon}^{\prime \prime}$ and the proposition we obtain:

$$
\gamma_{\varepsilon / Q}^{\prime \prime}\left([g]^{p} \omega\right) \leq \gamma_{\varepsilon / Q}^{\prime \prime}(\omega)+p \varepsilon / Q \leq \gamma_{\varepsilon / Q^{2}}^{\prime}(\omega)+p \varepsilon / Q .
$$

But on the other hand the proposition shows:

$$
\gamma_{\varepsilon / Q}^{\prime \prime}\left([g]^{p} \omega\right) \geq \gamma_{\varepsilon}^{\prime}\left([g]^{p} \omega\right)
$$

This shows (2.29).

For the last statement we remark that it is true for $[c]$, if there is an $h$ such that the statement is true for $[h c]$. Indeed this follows from (2.32). Therefore it suffices to assume that 
$c=f^{m} g$, where $g$ has no common divisor with $f$. This case is easily deduced from (2.29) and (2.30).

\section{Comparison with Monsky-Washnitzer cohomology}

Let $B / k$ be a finitely generated, smooth algebra over a perfect field $k$ of $\operatorname{char} p>0$. Let $\widetilde{B}^{\dagger}$ be the weak completion (in the sense of [14]) of a smooth finitely generated $W(k)$-algebra $\widetilde{B}$ lifting $B$. To begin this section we prove the existence of a map $\sigma: \widetilde{B}^{\dagger} \rightarrow W^{\dagger}(B)$ which we call an overconvergent Witt lift. It depends on a choice of Frobenius lift $F$ and is the same as the map $t_{F}: \widetilde{B}^{\dagger} \rightarrow W(B)$ described in [8]. We must prove that this map has image in $W^{\dagger}(B)$. We do this first for the case of a polynomial algebra (and any choice of Frobenius lift), and deduce the general result easily by functoriality.

Proposition 3.1. - Let $A=k\left[T_{1}, \ldots, T_{d}\right]$ and $\widetilde{A}^{\dagger}=W(k)\left\langle T_{1}, \ldots, T_{d}\right\rangle^{\dagger}$. Fix a Frobenius lift $F$ on $\widetilde{A}^{\dagger}$. Then the map $t_{F}$ defined in [8] p. 509 (and recalled below) has image in $W^{\dagger}(A)$.

Proof. - Let $a \in \widetilde{A}^{\dagger}$ have the form

$$
\sum_{k \in \mathbb{N}^{d}} \alpha_{k} T_{1}^{k_{1}} \cdots T_{d}^{k_{d}}
$$

For $\varepsilon>0$, we define a Gauss norm on $\widetilde{A}^{\dagger}$ by

$$
\gamma_{\varepsilon}(a)=\inf _{k}\left\{\operatorname{ord}_{p} \alpha_{k}-\varepsilon|k|\right\} .
$$

We define

$$
W^{\dagger}\left(\widetilde{A}^{\dagger}\right):=\left\{\left(a_{0}, a_{1}, \ldots\right) \in W\left(\widetilde{A}^{\dagger}\right) \mid m+\gamma_{\frac{\varepsilon}{p^{m}}}\left(a_{m}\right) \geq C, \text { for some } \varepsilon>0, C \in \mathbb{R}\right\} .
$$

The projection map pr : $W\left(\widetilde{A}^{\dagger}\right) \rightarrow W(A)$ induces a map $W^{\dagger}\left(\widetilde{A}^{\dagger}\right) \rightarrow W^{\dagger}(A)$.

For $x \in W\left(\widetilde{A}^{\dagger}\right)$, write $x=\left(a_{0}, a_{1}, \ldots\right)$ and let $w_{m}(x) \in \widetilde{A}^{\dagger}$ denote the $m$-th ghost component. Then we find

$$
m+\gamma_{\frac{\varepsilon}{p^{m}}}\left(a_{m}\right) \geq C \Longleftrightarrow \gamma_{\frac{\varepsilon}{p^{m}}}\left(w_{m}(x)\right) \geq C .
$$

The map $t_{F}$ is defined as the composition

$$
\widetilde{A}^{\dagger} \stackrel{s_{F}}{\rightarrow} W\left(\widetilde{A}^{\dagger}\right) \stackrel{\text { pr }}{\rightarrow} W(A)
$$

where for any $a \in \widetilde{A}^{\dagger}, s_{F}(a)$ is the unique element with ghost components $\left(a, F(a), F^{2}(a), \ldots\right)$. We claim that for any $a \in \widetilde{A}^{\dagger}$, there exist $\varepsilon, C$ with $\gamma_{\frac{\varepsilon}{p^{m}}}\left(F^{m}(a)\right) \geq C$ for all $m$. From the definition of $s_{F}$ and the above equivalence, this will immediately show that $s_{F}(a) \in W^{\dagger}\left(\widetilde{A}^{\dagger}\right)$, and so by the remark in our first paragraph, $t_{F}(a) \in W^{\dagger}(A)$.

Abbreviate $T$ for $\left(T_{1}, \ldots, T_{d}\right)$. Write $F\left(T_{i}\right)=T_{i}^{p}+p f_{i}(T)$ for each $i$. We can find $\varepsilon$ sufficiently small such that $\gamma_{\varepsilon}\left(f_{i}(T)\right)>-1$ for each $i$, and hence $\gamma_{\varepsilon}\left(p f_{i}(T)\right)>0$ for each $i$. From now on abbreviate $u_{i}:=p f_{i}(T)$.

Assume $\gamma_{\varepsilon}(a) \geq C$. For $k \in \mathbb{N}^{d}$, let $\partial_{k} a$ denote the partial derivative $\frac{\partial^{k_{1}}}{\partial T_{1}^{k_{1}}} \cdots \frac{\partial^{k_{d}}}{\partial T_{d}^{k_{d}}} a$. It is clear that $\gamma_{\varepsilon}\left(\frac{1}{k !} \partial_{k} a\right) \geq \gamma_{\varepsilon}(a) \geq C$. It is also clear that for any $h \in \widetilde{A}^{\dagger}$,

$$
\gamma_{\frac{\varepsilon}{p}}\left(h\left(T_{1}^{p}, \ldots, T_{d}^{p}\right)\right)=\gamma_{\varepsilon}\left(h\left(T_{1}, \ldots, T_{d}\right)\right) .
$$


Assuming still $\gamma_{\varepsilon}(a) \geq C$, we prove $\gamma_{\frac{\varepsilon}{p}}(F(a)) \geq C$. The result $\gamma_{\frac{\varepsilon}{p^{m}}}\left(F^{m}(a)\right) \geq C$ then follows by induction. We compute

$$
\begin{aligned}
F(a) & =a\left(T_{1}^{p}+u_{1}, \ldots, T_{d}^{p}+u_{d}\right) \\
& =\sum \frac{1}{k !} \partial_{k} a\left(T_{1}^{p}, \ldots, T_{d}^{p}\right) u^{k} ;
\end{aligned}
$$

so,

$$
\gamma_{\frac{\varepsilon}{p}}(F(a)) \geq \inf \left\{\gamma_{\frac{\varepsilon}{p}}\left(\frac{1}{k !} \partial_{k} a\left(T_{1}^{p}, \ldots, T_{d}^{p}\right)\right)+\gamma_{\frac{\varepsilon}{p}}\left(u^{k}\right)\right\} \geq C,
$$

as required.

Proposition 3.2. - For $B / k$ a smooth, finitely generated algebra with lift $\widetilde{B}^{\dagger}$ and Frobenius lift $F^{\prime}$, the map

$$
t_{F^{\prime}}: \widetilde{B}^{\dagger} \rightarrow W(B)
$$

has image in $W^{\dagger}(B)$.

Proof. - Take a surjective map from a polynomial algebra $\phi: A \rightarrow B$ and a lift of Frobenius $F$ on $\widetilde{A}^{\dagger}$ inducing $F^{\prime}$. Then the result follows from the functoriality of the map $t_{F}$ and the fact that the natural projection $W(A) \rightarrow W(B)$ sends $W^{\dagger}(A) \rightarrow W^{\dagger}(B)$.

Let $B / k$ be a finitely generated, smooth algebra over a perfect field $k$ of char $p>0$. We have just shown that $B$ admits an overconvergent Witt lift:

$$
\sigma: \widetilde{B}^{\dagger} \rightarrow W^{\dagger}(B)
$$

If we restrict $\sigma$ to the smooth $W(k)$-algebra $\widetilde{B}$ lifting $B$, we obtain an induced map

$$
\left.\sigma\right|_{\widetilde{B}}: \widetilde{B} \rightarrow W^{\dagger}(B)
$$

which we will call the underlying Witt lift associated to $\sigma$. Conversely, if we assume that $B$ admits a Witt lift, $\sigma: \widetilde{B} \rightarrow W(B)$ such that image $(\sigma) \subseteq W^{\dagger}(B)$, then $\sigma$ extends canonically to the weak completion of $\widetilde{B}$, i.e. to an overconvergent Witt lift

$$
\sigma: \widetilde{B}^{\dagger} \rightarrow W^{\dagger}(B)
$$

because $W^{\dagger}(B)$ is weakly complete (Proposition 2.28 in [5]). We derive from this a map of complexes, also denoted by $\sigma$

$$
\Omega_{\widetilde{B}^{\dagger} / W(k)} \rightarrow W^{\dagger} \Omega_{B / k}^{\bullet} \subset W \Omega_{B / k}^{\bullet}
$$

If $\hat{B}$ denotes the $p$-adic completion of $\widetilde{B}$ we also have a map

$$
\lim _{\leftarrow} \Omega_{\widetilde{B}_{n} / W_{n}(k)}^{\bullet}=: \Omega_{\hat{B} / W(k)}^{\bullet} \rightarrow W \Omega_{B / k}^{\bullet} .
$$

In the following we show that $\sigma$ in (3.4) is a quasi-isomorphism if $B$ is finite étale and monogenic over a localized polynomial algebra $A_{f}=k\left[T_{1}, \ldots, T_{n}\right]_{f}$.

Let $\widetilde{f} \in \widetilde{A}:=W(k)\left[T_{1}, \ldots, T_{n}\right]$ be a lifting of $f$ and $\widetilde{A}_{\widetilde{f}}:=W(k)\left[T_{1}, \ldots, T_{n}\right]_{\widetilde{f}} . B$ lifts to a finite étale extension $\widetilde{B}$ over $\widetilde{A}_{\widetilde{f}}$. If $B=A_{f}[x]$, then $\widetilde{B}=\widetilde{A}_{\widetilde{f}}[x]$. We write $u=[x]$ for the Teichmüller representative of $x$ in $W(B)$. Consider the canonical map

$$
\sigma: \widetilde{B} \rightarrow W^{\dagger}(B)=W^{\dagger}\left(A_{f}\right)[u]
$$


which extends the canonical map $\widetilde{A}_{\widetilde{f}} \rightarrow W^{\dagger}\left(A_{f}\right)$. The existence of $\sigma$ is derived from Hensel's lemma [5] Proposition 2.30. Hence $B$ has a canonical overconvergent Witt lift. Let $\widetilde{B}^{\dagger}, \widetilde{A}_{\widetilde{f}}^{\dagger}$ be the weak completions of $\widetilde{B}, \widetilde{A}_{\widetilde{f}}$. Then $\widetilde{B}^{\dagger}=\widetilde{A}_{\widetilde{f}}^{\dagger}[x]$ is finite étale over $\widetilde{A}_{\widetilde{f}}^{\dagger}$. Using Proposition 1.9 we see that $\sigma$ extends to a comparison map

$$
\sigma: \Omega_{\widetilde{B}^{\dagger} / W(k)}=\widetilde{B}^{\dagger} \bigotimes_{\widetilde{A}_{\tilde{f}}^{\dagger}} \Omega_{\widetilde{A}_{\tilde{f}}^{\dagger} / W(k)}^{\bullet} \rightarrow W^{\dagger} \Omega_{B / k}^{\bullet}=\bigoplus_{i=0}^{m-1} W^{\dagger} \Omega_{A_{f} / k}^{\bullet} x^{i}
$$

(here $m=\left[B: A_{f}\right]$ ).

We want to show that $\sigma$ is a quasi-isomorphism. First we treat the special case $B=A_{f}=k\left[T_{1}, \ldots, T_{n}\right]_{f}$. So we need to show:

$$
\sigma: \Omega_{\widetilde{A}_{\tilde{f}}^{\dagger} / W(k)} \rightarrow W^{\dagger} \Omega_{A_{f} / k}^{\bullet} \text { is a quasi-isomorphism. }
$$

We also consider $\widetilde{f}_{l}=\operatorname{image}(\widetilde{f})$ in $W_{l}(k)\left[T_{1}, \ldots, T_{n}\right]=: \widetilde{A}_{l}$. The $\widetilde{A}_{l}$-module structure in $W_{l} \Omega_{A / k}^{\bullet}$ respects the decomposition

$$
W_{l} \Omega_{A / k}^{\bullet}=W_{l} \Omega_{A / k}^{\bullet, \text { int }} \oplus W_{l} \Omega_{A / k}^{\bullet \text {,frac }}
$$

into integral and fractional part. This follows from [11] Lemma 4.

Hence we have a direct sum decomposition

$$
\begin{aligned}
W_{l} \Omega_{A_{f} / k}^{\bullet} & \cong \widetilde{A}_{l}\left[\frac{1}{\widetilde{f}_{l}}\right] \underset{\widetilde{A}_{l}}{\otimes} W_{l} \Omega_{A / k}^{\bullet} \\
& \cong \widetilde{A}_{l}\left[\frac{1}{\widetilde{f}_{l}}\right] \underset{\widetilde{A}_{l}}{\otimes} W_{l} \Omega_{A / k}^{\bullet, \text { int }} \oplus \widetilde{A}_{l}\left[\frac{1}{\widetilde{f}_{l}}\right] \underset{\widetilde{A}_{l}}{\otimes} W_{l} \Omega_{A / k}^{\bullet, \text { frac }}
\end{aligned}
$$

where the first isomorphism follows from the étale base change and the isomorphism

$$
W_{l}(A) \underset{\widetilde{A}_{l}}{\bigotimes} \widetilde{A}_{l}\left[\frac{1}{\widetilde{f}_{l}}\right] \cong W_{l}\left(A_{f}\right)
$$

When taking inverse limits, we put

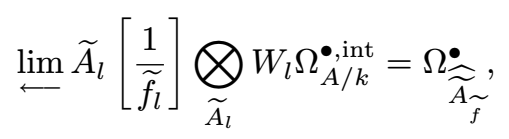

where $\widehat{\widetilde{A}}_{\widetilde{f}}$ is the $p$-adic completion of $\widetilde{A}_{\widetilde{f}}$. Then (3.6) yields a direct sum decomposition

$$
W \Omega_{A_{f} / k}^{\bullet} \cong W \Omega_{A_{f} / k}^{\bullet \text { int }} \oplus W \Omega_{A_{f} / k}^{\bullet \text { frac }}
$$

into two parts which we denote again by the integral and fractional part. We can identify $W \Omega_{A_{f} / k}^{\bullet \text {,int }}$ with $\Omega_{\underset{A_{\tilde{f}}}{\stackrel{\sim}{\sim}} \text { and we know that } W \Omega_{A_{f} / k}^{\bullet \text {,frac }}}$ is acyclic. With regards to $W^{\dagger} \Omega_{A_{f} / k}^{\bullet}$ we apply Proposition 1.3 and the remark after Proposition 1.3:

Any $z \in W^{\dagger} \Omega_{A_{f} / k}^{\bullet}$ can be written as a convergent series

$$
z=\sum_{l=0}^{\infty} \frac{1}{[f]^{r_{l}}} \eta_{l}
$$


where $\eta_{l}$ is a finite sum of basic Witt differentials $\eta_{l}^{(t)}$, such that there are real numbers $C$ and $\epsilon>0$ with

$$
\gamma_{\epsilon}\left(\eta_{l}\right)-\epsilon r_{l} \geq C
$$

The supremum over all $C$ for all possible representations of $z$ is by definition $\gamma_{\epsilon}(z)$, the Gauss norm on the localization.

We can also define an order function on $W \Omega_{A_{f} / k}^{\bullet}$ by considering representations of $z$ of the form

$$
z=\sum_{l=0}^{\infty} \frac{1}{\tilde{f}^{r_{l}}} \tau_{l} .
$$

We call $z$ convergent with radius $\epsilon$ with respect to $\tilde{f}$ if there are a representation and a constant $C \in \mathbb{R}$, such that

$$
\gamma_{\epsilon}\left(\tau_{l}\right)-\epsilon r_{l} \geq C
$$

We denote the supremum over all $C$ for all possible representations by $\gamma_{\epsilon}^{(\tilde{f})}(z)$. We will also express the last condition of convergence a little differently: We extend the function $\gamma_{\epsilon}$ to $W \Omega_{A / k}^{\bullet}[1 / \tilde{f}]$ as follows:

$$
\tilde{\gamma}_{\epsilon}\left(\omega / \tilde{f}^{k}\right)=\gamma_{\epsilon}(\omega)-k \gamma_{\epsilon}(\tilde{f})
$$

If $z=\sum_{l} z_{l}$ with $z_{l} \in W \Omega_{A / k}^{\bullet}[1 / \tilde{f}]$, and if we denote by $k_{l}$ the denominator of $z_{l}$ in this localization, it is easy to see that $\gamma_{\epsilon}^{(\tilde{f})}(z)$ is the supremum over all constants $C$ such that for a suitable representation $z=\sum z_{\alpha}$ we have

$$
k_{\alpha} \leq \frac{1}{\varepsilon(1+\operatorname{deg} \widetilde{f})}\left(\widetilde{\gamma}_{\varepsilon}\left(z_{\alpha}\right)+C\right) .
$$

We will prove that the notions of overconvergence and overconvergence with respect to $\tilde{f}$ are the same. We start with representations (3.8) such that (3.9) holds. We write

$$
\tilde{f}=[f]-\rho .
$$

It is enough to consider the case where $\epsilon$ is small enough. Therefore we may assume that $\gamma_{\epsilon}(\rho) \geq \epsilon$. We show that $z$ is convergent with respect to $\gamma_{\epsilon}$ :

$$
\begin{aligned}
\frac{1}{\widetilde{f}} & =\frac{1}{[f]} \frac{1}{1-\frac{1}{[f]} \rho} \\
& =\frac{1}{[f]}\left(1+\sum_{n \geq 1} \frac{1}{[f]^{n}}(\rho)^{n}\right) \\
& =\sum_{n \geq 1} \frac{1}{[f]^{n}}(\rho)^{n-1} .
\end{aligned}
$$

Then $\frac{1}{\widetilde{f}^{k}}=\sum_{m \geq k} a_{m} \frac{1}{[f]^{m}}(\rho)^{m-k}$ with $a_{m} \in \mathbb{Z}$.

Then we find

$$
\gamma_{\epsilon}\left(\sum_{l} \frac{1}{\left[\tilde{f}^{r_{l}}\right]} \tau_{l}\right)=\gamma_{\epsilon}\left(\sum_{l} \sum_{m \geq r_{l}} a_{m} \frac{1}{[f]^{m}} \tau_{l} \rho^{m-r_{l}}\right)
$$

$4^{\mathrm{e}}$ SÉRIE - TOME $44-2011$ - No 2 
We give an estimation for each summand separately:

$$
\begin{aligned}
\gamma_{\epsilon}\left(a_{m} \frac{1}{[f]^{m}} \tau_{l} \rho^{m-r_{l}}\right) & \geq \gamma_{\epsilon}\left(\tau_{l} \rho^{m-r_{l}}\right)-\epsilon m \geq \gamma_{\epsilon}\left(\tau_{l}\right)+\breve{\gamma}_{\epsilon}\left(\rho^{m-r_{l}}\right)-\epsilon m \\
& \geq \gamma_{\epsilon}\left(\tau_{l}\right)-r_{l} \epsilon-\left(m-r_{l}\right) \epsilon+\left(m-r_{l}-1\right) \gamma_{\epsilon}(\rho)+\breve{\gamma}_{\epsilon}(\rho) .
\end{aligned}
$$

The last inequality holds by [5] (2.22). Since $\gamma_{\epsilon}(\rho) \geq \epsilon$ we conclude:

$$
\gamma_{\epsilon}\left(a_{m} \frac{1}{[f]^{m}} \tau_{l} \rho^{m-r_{l}}\right) \geq C+\left(m-r_{l}-1\right)\left(\gamma_{\epsilon}(\rho)-\epsilon\right)-\epsilon+\breve{\gamma}_{\epsilon}(\rho) \geq C-\epsilon(1+\operatorname{deg} f) .
$$

The last inequality was explained at the end of the proof of Corollary 0.13 . Finally we obtain

$$
\gamma_{\epsilon}(z) \geq \gamma_{\epsilon}^{(\tilde{f})}(z)-\epsilon(1+\operatorname{deg} f) .
$$

If we interchange the roles of $[f]$ and $\tilde{f}$ in the argument above we see that:

$$
\gamma_{\epsilon}^{(\tilde{f})}(z) \geq \gamma_{\epsilon}(z)-\epsilon(1+\operatorname{deg} f) .
$$

The Gauss norms $\gamma_{\epsilon}^{(\tilde{f})}$ are appropriate to study overconvergence on the integral and fractional part of $W \Omega_{A_{f} / k}$ separately. More precisely let $z \in W \Omega_{A_{f} / k}$ and let $z=z_{1}+z_{2}$ according to the decomposition (3.7). We have just seen that $\gamma_{\epsilon}(z)>-\infty \Leftrightarrow \gamma_{\epsilon}^{(\tilde{f})}(z)>-\infty$ for small $\epsilon$. We claim that

$$
\gamma_{\epsilon}^{(\tilde{f})}(z)>-\infty \text { implies } \gamma_{\epsilon}^{(\tilde{f})}\left(z_{1}\right)>-\infty \text { and } \gamma_{\epsilon}^{(\tilde{f})}\left(z_{2}\right)>-\infty \text {. }
$$

Let $\gamma_{\epsilon}^{(\tilde{f})}(z) \geq C$; then there exists a representation

$$
z=\sum_{l=0}^{\infty} \frac{1}{\tilde{f}^{r_{l}}} \tau_{l}
$$

such that

$$
\gamma_{\epsilon}\left(\tau_{l}\right)-\epsilon r_{l} \geq C
$$

Let $\tau_{l}=\tau_{l}^{1}+\tau_{l}^{2}$ be the decomposition in integral and fractional part. Then

$$
z_{1}=\sum_{l=0}^{\infty} \frac{1}{\tilde{f}^{r_{l}}} \tau_{l}^{1} \text { and } z_{2}=\sum_{l=0}^{\infty} \frac{1}{\tilde{f}^{r_{l}}} \tau_{2}^{1}
$$

As $\gamma_{\epsilon}\left(\tau_{l}\right)=\min \left\{\gamma_{\epsilon}\left(\tau_{l}^{1}\right), \gamma_{\epsilon}\left(\tau_{l}^{2}\right)\right\}$ the claim follows. Hence we obtain a direct sum decomposition

$$
W^{\dagger} \Omega_{A_{f} / k}^{\bullet}=W^{\dagger} \Omega_{A_{f} / k}^{\bullet \text { int }} \bigoplus W^{\dagger} \Omega_{A_{f} / k}^{\bullet, \text { frac }} .
$$

We will also consider the truncated Gauss norms $\gamma_{\epsilon}^{(\tilde{f})}[t]$ on

$$
\begin{aligned}
\widetilde{A}_{t+1}\left[\frac{1}{\tilde{\widetilde{f}}}\right] \otimes_{\widetilde{A}_{t+1}} W_{t+1} \Omega_{A / k}^{\bullet} & \\
& =\widetilde{A}_{t+1}\left[\frac{1}{\widetilde{f}}\right] \otimes_{\widetilde{A}_{t+1}} W_{t+1} \Omega_{A / k}^{\bullet, \text { int }} \oplus \widetilde{A}_{t+1}\left[\frac{1}{\widetilde{f}}\right] \otimes_{\widetilde{A}_{t+1}} W_{t+1} \Omega_{A / k}^{\bullet, \text { frac }} .
\end{aligned}
$$

We can define the Gauss norm $\widetilde{\gamma}_{\varepsilon}$ on $\widetilde{A}_{t+1}\left[\frac{1}{\widetilde{f}}\right] \underset{\widetilde{A}_{t+1}}{\bigotimes W_{t+1} \Omega_{A / k}^{\bullet, \text { frac }}}$ in the same way as before. We fix $\varepsilon>0$ and $C \in \mathbb{R}$. 
We define $W_{t+1} \Omega_{A_{f} / k}^{\bullet, \text { frac }, C, C}$ as the set of finite sums $\sum_{k} \frac{\eta_{k}}{\widetilde{f}^{k}} \in W_{t+1} \Omega_{A_{f} / k}^{\bullet \text { frac }}=$ $\widetilde{A}_{t+1}\left[\frac{1}{\widetilde{f}}\right] \underset{\widetilde{A}_{t+1}}{\bigotimes} W_{t+1} \Omega_{A / k}^{\mathrm{frac}}$ satisfying the following. Let $K_{0}$ be the largest integer divisible by $p$ such that

$$
K_{0} \leq \frac{1}{\varepsilon(1+\operatorname{deg} \widetilde{f})}\left(\widetilde{\gamma}_{\varepsilon}\left(\frac{w}{\widetilde{f}_{l}^{k}}\right)+C\right) .
$$

Then we require the following two conditions:

(i) $K_{0} \geq 0$

(ii) $k \leq K_{0}$.

We know that the complex $\widetilde{A}_{t+1}\left[\frac{1}{\widetilde{f}}\right] \underset{\widetilde{A}_{t+1}}{\bigotimes} W_{t+1} \Omega_{A / k}^{\bullet, \text { frac }}$ is acyclic. We show that for $\varepsilon>0$ sufficiently small $W_{t+1} \Omega_{A_{f} / k}^{\mathrm{frac}, C, C}$ is acyclic.

Let us assume that $f$ is regular in the variable $T_{1}$. Let $c \in A_{f}$. Then $c$ has a unique reduced representation:

$$
c=\sum_{l} a_{l} / f^{l}
$$

where $a_{l} \in A$. We write $a=\sum \alpha_{k} T^{k} \in A$, with $\alpha_{k} \in k$, and we set $\tilde{a}=\sum\left[\alpha_{k}\right][T]^{k} \in W(A)$. Then we define

$$
\tilde{c}=\sum_{l} \tilde{a}_{l} / \tilde{f}^{l}
$$

This is an integral element in $W\left(A_{f}\right)$. In the following we consider still another admissible Gauss norm on $W_{t+1} \Omega_{A_{f} / k}$. Let $\omega \in W_{t+1} \Omega_{A_{f} / k}$. Then we consider all possible expressions of the type:

$$
\omega=\sum_{l} \eta_{l} / \tilde{f}^{l p}, \quad \eta_{l} \in W_{t+1} \Omega_{A / k} .
$$

We forget our old notation and denote by $\gamma_{\varepsilon}^{\prime}[t](\omega)$ the maximum over all possible numbers

$$
\min \left\{\gamma_{\varepsilon}[t]\left(\eta_{l}\right)-\varepsilon l p\right\} .
$$

It is easy to see that the condition $\gamma_{\epsilon}^{\prime}[t](\omega) \geq C$ for $\omega \in W_{t+1} \Omega_{A_{f} / k}^{\text {frac }}$ is equivalent to condition $\omega \in W_{t+1} \Omega_{A_{f} / k}^{\mathrm{frac}, C}$.

We should remark that $\gamma_{\varepsilon}^{\prime}[1]$ coincides with the formerly defined function. As before we define a modified $\hat{\gamma}_{\varepsilon}^{\prime}[t]$. Then we have $\hat{\gamma}_{\epsilon}^{\prime}[t]=\gamma_{\epsilon}^{(\tilde{f})}[t]$

We find the equalities:

$$
\hat{\gamma}_{\varepsilon}^{\prime}[t](\tilde{c})=\hat{\gamma}_{\varepsilon}^{\prime}(c), \quad \gamma_{\varepsilon}^{\prime}[t](\tilde{c})=\gamma_{\varepsilon}^{\prime}(c) .
$$

Indeed we verify the first equation as follows: By the representation (3.13) we find:

$$
\hat{\gamma}_{\varepsilon}^{\prime}[t](\tilde{c}) \geq \min \left\{\gamma_{\varepsilon}[t]\left(\tilde{a}_{l}\right)-\varepsilon l\right\}=\min _{l}\left\{\gamma_{\varepsilon}\left(a_{l}\right)-\varepsilon l\right\}=\hat{\gamma}_{\varepsilon}^{\prime}(c)=\hat{\gamma}_{\varepsilon}^{\prime}[1](\tilde{c}) .
$$

The other inequality is obvious. 
LeMmA 3.14. - Each $\omega \in W_{t+1} \Omega_{A_{f} / k}$ has a unique representation:

$$
\omega=\sum{ }^{F} \tilde{c}_{i}^{(n)} \omega_{i}^{(n)}
$$

This decomposition respects the non integral and the integral part, i.e. if $\omega$ is integral (resp. non integral) then all $\omega_{i}^{(n)}$ are integral (respectively non integral). For the Gauss norm we have:

$$
\gamma_{\varepsilon}^{\prime}[t](\omega)=\min \left\{p \hat{\gamma}_{\varepsilon}^{\prime}\left(c_{i}^{n}\right)+\gamma_{\varepsilon}\left(\omega_{i}^{(n)}\right)\right\}
$$

Proof. - The same as that of Proposition 2.20: The Lemmas 2.11 and 2.13 continue to hold with ${ }^{F} \tilde{c}_{i}^{(n)}$ in place of ${ }^{F}\left[c_{i}^{(n)}\right]$, because the action of both elements is the same on the graded part $G^{n}$. We need to verify that for fixed $n$ :

$$
\begin{aligned}
\gamma_{\varepsilon}^{\prime}[t]\left(\sum_{i}{ }^{F} \tilde{c}_{i}^{(n)} \omega_{i}^{(n)}\right) & =\min \left\{\gamma_{\varepsilon}^{\prime}[t]\left({ }^{F} \tilde{c}_{i}^{(n)}\right)+\gamma_{\varepsilon}\left(\omega_{i}^{(n)}\right)\right\} \\
& =\min \left\{p \hat{\gamma}_{\varepsilon}\left(c_{i}^{(n)}\right)+\gamma_{\varepsilon}\left(\omega_{i}^{(n)}\right)\right\} .
\end{aligned}
$$

It is clear from Lemma 2.13 that this is true for $\gamma_{\varepsilon}^{\prime}[n]$ in place of $\gamma_{\varepsilon}^{\prime}[t]$. We choose reduced representations:

$$
c_{i}^{(n)}=\sum_{l} a_{i, l}^{(n)} / f^{l}
$$

Then we find:

$$
\gamma_{\varepsilon}^{\prime}[t]\left(\sum_{i}{ }^{F} \tilde{c}_{i}^{(n)} \omega_{i}^{(n)}\right)=\gamma_{\varepsilon}^{\prime}[t]\left(\sum_{l}\left(\sum_{i}{ }^{F} \tilde{a}_{i, l}^{(n)} \omega_{i}^{(n)} / \tilde{f}^{p l}\right)\right) .
$$

From this we see that:

$$
\begin{aligned}
\gamma_{\varepsilon}^{\prime}[t]\left(\sum_{i} F^{F} \tilde{c}_{i}^{(n)} \omega_{i}^{(n)}\right) & \geq \min \left\{\gamma_{\varepsilon}[t]\left(\sum\left({ }^{F} \tilde{a}_{i, l}^{(n)} \omega_{i}^{(n)}\right)\right)-\varepsilon l p\right\} \\
& =\min \left\{\gamma_{\varepsilon}[t]\left({ }^{F} \tilde{a}_{i, l}^{(n)}\right)+\gamma_{\varepsilon}\left(\omega_{i}^{(n)}\right)-\varepsilon l p\right\} \\
& =\min \left\{\gamma_{\varepsilon}^{\prime}[t]\left({ }^{F} \tilde{c}_{i}^{(n)}\right)+\gamma_{\varepsilon}\left(\omega_{i}^{(n)}\right)\right\}
\end{aligned}
$$

This shows the Equation (3.16) because $\gamma_{\varepsilon}^{\prime}[t] \leq \gamma_{\varepsilon}^{\prime}[n]$. The rest of the proof of the lemma is the same.

Proposition 3.17. - Let $\varepsilon \in \mathbb{R}$ be sufficiently small. Let $\omega \in W_{t+1} \Omega_{A_{f} / k}$ be a closed Witt differential in the non integral part such that $\gamma_{\varepsilon}^{\prime}(\omega) \geq C$. Then $\omega=d \eta$, where $\eta \in W_{t+1} \Omega_{A_{f} / k}$ is a Witt differential in the non integral part, such that $\gamma_{\varepsilon}^{\prime}(\eta) \geq C$.

Proof. - The problem does not change if we make a finite extension of the base field $k$. Therefore we may assume that $f$ is regular in $T_{1}$ as above.

Consider the residue class $\bar{\omega} \in W_{2} \Omega_{A_{f} / k}$ of $\omega$. This is a closed form in the fractional part, i.e. it is contained in the module:

$$
\left(d V^{n} P^{l-1}\right)_{f} \oplus\left(p d V^{n-1} P^{l-1}\right)_{f} \oplus \cdots \oplus\left(p^{n-1} d V P^{l}\right)_{f}
$$

for $n=2$. This means that all basic Witt differentials $\omega_{i}^{(1)}$, which appear in the decomposition (3.15) must be of the form $\omega_{i}^{(1)}=d \eta_{i}^{(1)}$ for some primitive basic Witt differential $\eta_{i}^{(1)}$, such that $\gamma_{\varepsilon}\left(\omega_{i}^{(1)}\right)=\gamma_{\varepsilon}\left(\eta_{i}^{(1)}\right)$. We set:

$$
\eta(1)=\sum{ }^{F} \tilde{c}_{i}^{(1)} \eta_{i}^{(1)}
$$


Clearly $\gamma_{\varepsilon}^{\prime}(\eta(1))=\min \left\{p \hat{\gamma}_{\varepsilon}^{\prime}\left(c_{i}^{(1)}\right)+\gamma_{\varepsilon}\left(\omega_{i}^{(1)}\right)\right\} \geq \gamma_{\varepsilon}(\omega)$.

We will verify that for small $\varepsilon$ :

$$
\gamma_{\varepsilon}^{\prime}(d \eta(1)) \geq \gamma_{\varepsilon}^{\prime}(\omega)
$$

Then we consider $\omega(1)=\omega-d \eta(1)$. We conclude that $\gamma_{\varepsilon}^{\prime}\left(\omega(1) \geq \gamma_{\varepsilon}^{\prime}(\omega)\right.$ and that $\omega(1) \in \mathrm{Fil}^{2} W_{t+1} \Omega_{A_{f} / k}$. Then we expand $\omega(1)$ in the form (3.15) and consider the reduction in $W_{3} \Omega_{A_{f} / k}$. We apply the same argument and find $\eta(2)$ with $\gamma_{\varepsilon}^{\prime}(\eta(2)) \geq \gamma_{\varepsilon}^{\prime}(\omega(1))$ and $\gamma_{\varepsilon}^{\prime}(d \eta(2)) \geq \gamma_{\varepsilon}^{\prime}(\omega(1))$. Continuing we obtain:

$$
\omega=d \eta(1)+d \eta(2)+d \eta(3)+\cdots
$$

This proves the result if we verify (3.18).

We set $C=\gamma_{\varepsilon}^{\prime}(\omega)$. By definition ${ }^{F} \tilde{c}_{i}^{(n)}$ is a sum of expressions $[u]^{p} / \tilde{f}^{l p}$ such that:

$$
p \gamma_{\varepsilon}([u])-\varepsilon l p+\gamma_{\varepsilon}\left(\eta_{i}^{(1)}\right) \geq C .
$$

Here $u$ is a monomial in the variables $T$. We have to verify that

$$
\gamma_{\varepsilon}^{\prime}\left(d\left([u]^{p} \eta_{i}^{(1)} / \tilde{f}^{l p}\right)\right) \geq C .
$$

We write:

$$
d\left([u]^{p} \eta_{i}^{(1)} / \tilde{f}^{l p}\right)=\left(d\left([u]^{p} \eta_{i}^{(1)}\right)\right) / \tilde{f}^{l p} \stackrel{+}{-} l p\left([u]^{p} \eta_{i}^{(1)} \tilde{f}^{p-1} d \tilde{f}\right) / \tilde{f}^{(l+1) p} .
$$

Clearly $\gamma_{\varepsilon}^{\prime}$ of the first summand is greater than $C$. We have:

$$
\gamma_{\varepsilon}^{\prime}\left(p l[u]^{p} \eta_{i}^{(1)} \tilde{f}^{p-1} d \tilde{f} / \tilde{f}^{(l+1) p}\right) \geq p \gamma_{\varepsilon}([u])-\varepsilon(l+1) p+\gamma_{\varepsilon}\left(\omega_{i}^{(1)}\right)+p \gamma_{\varepsilon}(\tilde{f})+1 .
$$

The last expression is bigger $C$ if

$$
p \gamma_{\varepsilon}(\tilde{f})+1-p \varepsilon \geq 0 .
$$

But this is clearly fulfilled for small $\varepsilon$.

Hence $W_{t+1} \Omega_{A_{f} / k}^{\mathrm{frac}, \varepsilon}$ is acyclic. As the notions of overconvergence on $W \Omega_{A_{f} / k}$ and overconvergence with respect to $\tilde{f}$ are the same we can apply the remark preceding Corollary 2.24. We see that the complex $W^{\dagger} \Omega_{A_{f} / k}^{\mathrm{frac}, \varepsilon}$ consisting of elements $\omega \in W \Omega_{A_{f} / k}^{\mathrm{frac}}$ satisfying $\gamma_{\varepsilon}^{\prime}[t](\omega) \geq C$ for some $C$ independently of $t$ is exact as well. Hence

$$
W^{\dagger} \Omega_{A_{f} / k}^{\bullet \text { frac }}=\lim _{\varepsilon \rightarrow 0} W^{\dagger} \Omega_{A_{f} / k}^{\text {frac, } \varepsilon}
$$

is exact, as desired.

Now we can prove the following comparison result.

Theorem 3.19. - Let $f \in k\left[T_{1}, \ldots, T_{d}\right]=A$. Let $B$ be finite étale and monogenic over $A_{f}$.

Then the map $\sigma$, explicitly given in 3.5, of complexes

$$
\sigma: \Omega_{\widetilde{B}^{\dagger} / W(k)} \cong W^{\dagger} \Omega_{B / k}
$$

is a quasi-isomorphism.

4 ${ }^{\mathrm{e}}$ SÉRIE - TOME 44 - 2011 - No 2 
Proof. - We consider a lift $\tilde{A}_{\tilde{f}}$ of $A_{f}$ over $W(k)$ and a finite monogenic étale algebra $\tilde{B}$ over $\tilde{A}_{\tilde{f}}$ which lifts $B$. We write $\tilde{B}=\tilde{A}_{\tilde{f}}[x]$. We denote by $\widetilde{B}^{\dagger}$ the weak completion of $\tilde{B}$. By choosing a Frobenius on the weak completions we find morphisms

$$
\tilde{B} \rightarrow W(B), \quad \tilde{A}_{\tilde{f}} \rightarrow W\left(A_{f}\right) .
$$

The elements

$$
1, x, \ldots, x^{m-1}
$$

form a basis of the free $\tilde{A}_{\tilde{f}}$-module $\tilde{B}$. For any power $p^{n}$ the elements

$$
1, x^{p^{n}}, x^{2 p^{n}} \ldots, x^{(n-1) p^{n}}
$$

form also a basis of $\tilde{B}$ over $\tilde{A}_{\tilde{f}}$.

We have the isomorphism of modules (not of complexes):

$$
W \Omega_{B / k}=\tilde{B} \otimes_{\tilde{A}_{\tilde{f}}} W \Omega_{A_{f} / k}=\bigoplus_{i=0}^{m-1} x^{i p} W \Omega_{A_{f} / k} .
$$

Let $\gamma_{\epsilon}^{\prime}$ be the Gauss norms on $W \Omega_{A_{f} / k}$ considered in Lemma 3.14. We consider the product norms on the right hand side of 3.20 . We write $\omega \in W \Omega_{B / k}$ :

$$
\omega=\sum \eta_{j} x^{j p}
$$

Then we set

$$
\gamma_{\epsilon}(\omega)=\min \left\{\gamma_{\epsilon}^{\prime}\left(\eta_{j}\right)\right\}
$$

According to (3.20) we find:

$$
d x^{i p}=p i x^{i p-1} d x=\sum_{j=0}^{m-1} x^{j p} \vartheta_{i j},
$$

where the $\vartheta_{i j} \in \Omega_{\tilde{A}_{\tilde{f}} / W(k)} \subset W \Omega_{A_{f} / k}$ are integral differentials. We restrict our attention to small $\epsilon$. Then we may assume that

$$
\gamma_{\epsilon}^{\prime}\left(\vartheta_{i j}\right)>0
$$

This is possible because the $\vartheta_{i j}$ are divisible by $p$ and $\gamma_{\epsilon}(p)=1$. The last assumption ensures that

$$
\gamma_{\epsilon}(d \omega) \geq \gamma_{\epsilon}(\omega)
$$

We define the fractional part of $W \Omega_{B / k}$ :

$$
W \Omega_{B / k}^{\mathrm{frac}}=\tilde{B} \otimes_{\tilde{A}_{\tilde{f}}} W \Omega_{A_{f} / k}^{\mathrm{frac}} .
$$

This is a subcomplex of $W \Omega_{B / k}$. We denote by $W^{\dagger} \Omega_{B / k}^{\text {frac }}$ the overconvergent differentials in $W \Omega_{B / k}^{f r a c}$. By the decompositions (3.5), (3.7), and (3.11), it remains to show that this complex of overconvergent fractional differentials is acyclic.

From (3.20) we obtain decompositions for the filtrations:

$$
\mathrm{Fil}^{n} W \Omega_{B / k}^{\mathrm{frac}}=\bigoplus_{j=0}^{m-1} x^{j p} \mathrm{Fil}^{n} W \Omega_{A_{f} / k}^{\mathrm{frac}} .
$$

Consider a closed overconvergent Witt differential $\omega \in W \Omega_{B / k}^{\mathrm{frac}}$ :

$$
d \omega=0, \quad \gamma_{\epsilon}(\omega) \geq-C .
$$


We will show that $\omega=d \eta$ for $\eta \in W \Omega_{B / k}^{\text {frac }}$ with $\gamma_{\epsilon}(\eta) \geq-C$. This implies that the complex $W^{\dagger} \Omega_{B / k}^{\mathrm{frac}}$ is acyclic.

We note that $\omega \in \mathrm{Fil}^{1} W \Omega_{B / k}^{\mathrm{frac}}=W \Omega_{B / k}^{\mathrm{frac}}$. We set $\omega_{1}=\omega$. We construct inductively fractional differentials $\omega_{i}, \eta_{i} \in \mathrm{Fil}^{i} W \Omega_{B / k}^{\mathrm{frac}}$, such that $\gamma_{\epsilon}\left(\omega_{i}\right) \geq-C, \gamma_{\epsilon}\left(\eta_{i}\right) \geq-C$ and

$$
\omega_{i}=\omega_{i+1}+d \eta_{i}
$$

We consider $\omega_{i}$ modulo Fil ${ }^{i+1} W \Omega_{B / k}^{\text {frac }}$ i.e. as an element of $\operatorname{gr}^{i} W \Omega_{B / k}^{\text {frac }} \subset W_{i+1} \Omega_{B / k}^{\text {frac }}$. Then, using (3.22), we may write:

$$
\omega_{i}=\sum x^{j p}\left(V^{i} \sigma_{j}+d^{V^{i}} \rho_{j}\right)
$$

Since $\operatorname{gr}^{i} W \Omega_{B / k}^{\mathrm{frac}}$ is annihilated by $p$ we have

$$
0=d \omega_{i}=\sum x^{j p} d^{V^{i}} \sigma_{j}
$$

This shows that $V^{i} \sigma_{j}=0$, for $j=0, \ldots, m-1$. We find for the truncated norms:

$$
\min \left\{\gamma_{\epsilon}^{\prime}[i]\left(d^{V^{i}} \rho_{j}\right)\right\}=\gamma_{\epsilon}[i]\left(\omega_{i}\right) \geq-C .
$$

Using Proposition 3.17 we may assume after a possible modification of the $\rho_{j}$ that $\gamma_{\epsilon}^{\prime}[i]\left(V^{i} \rho_{j}\right) \geq-C$. We choose liftings $V^{i} \tilde{\rho}_{j} \in W \Omega_{A_{f} / k}^{\text {frac }}$, such that

$$
\gamma_{\epsilon}^{\prime}\left(V^{i} \tilde{\rho}_{j}\right)=\gamma_{\epsilon}^{\prime}[i]\left(V^{i} \rho_{j}\right) \geq-C .
$$

Since $d$ increases the product norm we find

$$
\gamma_{\epsilon}\left(d \sum x^{j p V^{i}} \tilde{\rho}_{j}\right) \geq \gamma_{\epsilon}\left(\sum x^{j p V^{i}} \tilde{\rho}_{j}\right) \geq-C .
$$

We set

$$
\eta_{i}=\sum x^{j p V^{i}} \tilde{\rho}_{j}, \quad \omega_{i+1}=\omega_{i}-d \eta_{i} .
$$

This ends the induction and the proof of the proposition.

For an arbitrary smooth algebra $A$, consider an overconvergent Witt lift

$$
\psi: \widetilde{A}^{\dagger} \rightarrow W^{\dagger}(A)
$$

which is uniquely determined by a lifting of the Frobenius to $\widetilde{A}^{\dagger}$. (Compare Proposition 3.2.) It induces a map of complexes, also denoted by $\psi$,

$$
\psi: \Omega_{\widetilde{A}^{\dagger} / W(k)} \rightarrow W^{\dagger} \Omega_{A / k} .
$$

Passing to cohomology we will prove the following comparison result.

Proposition 3.24. - Let $\kappa=\left\lfloor\log _{p} \operatorname{dim} A\right\rfloor$. Then the kernel and cokernel of the induced homomorphism

$$
\psi_{*}: H^{i}\left(\Omega_{\widetilde{A}^{\dagger} / W(k)}\right) \rightarrow H^{i}\left(W^{\dagger} \Omega_{A / k}\right)
$$

are annihilated by $p^{2 \kappa}$.

$4^{\text {e }}$ SÉRIE - TOME $44-2011$ - No 2 
Corollary 3.25. - (a) Let $\operatorname{dim} A<p$. Then $\psi_{*}$ is an isomorphism.

(b) In general, there is a (rational) isomorphism

$$
H_{\mathrm{MW}}^{*}(A / K) \cong H^{*}\left(W^{\dagger} \Omega_{A / k} \otimes_{W(k)} K\right)
$$

between Monsky-Washnitzer cohomology and overconvergent de Rham-Witt cohomology. (Here $K=W(k)\left[\frac{1}{p}\right]$.)

We will reduce the proof of the proposition to a local homotopy argument. The map $\psi$ induces a map of complexes of Zariski sheaves on $\operatorname{Spec} A$ :

$$
\widetilde{\psi}: \widetilde{\Omega}_{\widetilde{A}^{\dagger} / W(k)} \rightarrow W^{\dagger} \Omega_{\mathrm{Spec} A / k} .
$$

As $H_{\mathrm{Zar}}^{i}\left(\operatorname{Spec} A, \widetilde{\Omega}_{\widetilde{A}^{\dagger} / W(k)}^{d}\right)=H_{\mathrm{Zar}}^{i}\left(W^{\dagger} \Omega_{\operatorname{Spec} A / k}^{d}\right)=0$ for all $d \geq 0$ and all $i>0$ (Proposition 1.2 and [13] Lemma 7), we have

$$
\begin{aligned}
R \Gamma\left(\operatorname{Spec} A, \widetilde{\Omega}_{\widetilde{A}^{\dagger} / W(k)}^{\bullet}\right) & =\Omega_{\widetilde{A}^{\dagger} / W(k)}^{\bullet} \text { and } \\
R \Gamma\left(\operatorname{Spec} A, W^{\dagger} \Omega_{\text {Spec } A / k}\right) & =W^{\dagger} \Omega_{A / k},
\end{aligned}
$$

hence we can reconstruct $\psi$ from $\widetilde{\psi}$ by applying $R \Gamma\left(\operatorname{Spec} A\right.$, .). Let $\left\{U_{j}\right\}_{j}$ be a finite affine covering of Spec $A$ such that each $U_{j}$ is finite étale and monogenic over a localized polynomial algebra. By a result of Kedlaya [9], such a covering always exists. Let $U_{j}=\operatorname{Spec} B_{j}$ and $\widetilde{B}_{j}^{\dagger}$ the Monsky-Washnitzer lift of $B_{j}$. Then we consider the "localization" $\psi_{j}$ of $\psi$ to $U_{j}$ :

$$
\psi_{j}: \Omega_{\widetilde{B}_{j}^{\dagger} / W(k)} \rightarrow W^{\dagger} \Omega_{B_{j} / k}
$$

We compare the map $\psi_{j}$ with the explicitly given comparison map $\sigma$ in (3.5) from which we know it is a quasi-isomorphism and show the following.

Proposition 3.26. - The maps $p^{\kappa} \psi_{j}$ and $p^{\kappa} \sigma$ are homotopic, hence induce the same map on cohomology.

Before proving the proposition we finish the proof of Proposition 3.24. We know that the kernel and cokernel of $\left(p^{\kappa} \psi_{j}\right)_{*}$ are annihilated by $p^{\kappa}$. As $\operatorname{Ker}\left(\psi_{j}\right)_{*} \subseteq \operatorname{Ker}\left(p^{\kappa} \psi_{j}\right)_{*}$ and $\operatorname{Coker}\left(\psi_{j}\right)_{*}$ is a subquotient of $\operatorname{Coker}\left(p^{\kappa} \psi_{j}\right)_{*}, \operatorname{Ker}\left(\psi_{j}\right)_{*}$ and $\operatorname{Coker}\left(\psi_{j}\right)_{*}$ are annihilated by $p^{\kappa}$ as well.

Define $C^{\bullet}$ as the complex of Zariski sheaves obtained by taking the cokernel of $\widetilde{\psi}$. Then one has an exact sequence of complexes of Zariski sheaves

$$
0 \rightarrow \widetilde{\Omega}_{\widetilde{A}^{\dagger} / W(k)}^{\bullet} \rightarrow W^{\dagger} \Omega_{\operatorname{Spec} A / k}^{\bullet} \rightarrow C^{\bullet} \rightarrow 0 .
$$

The cohomology sheaves $H^{i}\left(C^{\bullet}\right)$ are annihilated by $p^{2 \kappa}$. Hence the map $C^{\bullet} \stackrel{p^{2 \kappa}}{\rightarrow} C^{\bullet}$ induces the zero map on cohomology. Therefore it is zero in the derived category. Applying the functor $R \Gamma$ we see that $R^{i} \Gamma\left(\operatorname{Spec} A, C^{\bullet}\right) \stackrel{p^{2 \kappa}}{\rightarrow} R^{i} \Gamma\left(\operatorname{Spec} A, C^{\bullet}\right)$ is the zero map. This finishes the proof of Proposition 3.24.

We now prove Proposition 3.26. It is implied by the following more general result. Let $B, C$ denote smooth $k$-algebras which are finite and étale over localized polynomial algebras, with smooth lifts $\widetilde{B}, \widetilde{C}$ and corresponding weak completions $\widetilde{B}^{\dagger}, \widetilde{C}^{\dagger}$. 
Proposition 3.27. - Let $\tilde{\phi}_{1}, \tilde{\phi}_{2}: \widetilde{B}^{\dagger} \rightarrow W^{\dagger}(C)$ denote two lifts of a map $\phi: B \rightarrow C$. Then the induced maps

$$
p^{\kappa} \tilde{\phi}_{1}, p^{\kappa} \tilde{\phi}_{2}: \Omega_{\widetilde{B}^{\dagger} / W(k)} \rightarrow W^{\dagger} \Omega_{C / k}
$$

are chain homotopic, where $\kappa=\left\lfloor\log _{p} \operatorname{dim} B\right\rfloor$.

We will closely follow the argument on pages 205-206 of [14].

Proof. - The chain homotopy we produce will factor through the following algebra.

Definition 3.28. - Denote by $D^{\prime \prime}(C)$ the differential graded algebra with $i$-th graded piece

$$
D^{\prime \prime}(C)^{i}=W^{\dagger} \Omega_{C / k}^{i}[[U]] \oplus W^{\dagger} \Omega_{C / k}^{i-1}[[U]] \wedge d U .
$$

Denote by $D^{\prime}(C)$ the sub-differential graded algebra of $D^{\prime \prime}(C)$ generated in degree zero by terms

$$
f=\sum_{i=0}^{\infty} U^{i} \omega_{i}
$$

for which $\omega_{i} \in p^{i-1} V W^{\dagger}(C)$ for $i \geq 1$ and such that there exist $\varepsilon, G$ with $\gamma_{\varepsilon}\left(\omega_{i}\right) \geq G$ for all $i$.

For such a term $f$, we define

$$
\gamma_{\varepsilon}(f)=\inf _{i}\left\{\gamma_{\varepsilon}\left(\omega_{i}\right)\right\}
$$

Note that $D^{\prime}(C)^{0}$ is an algebra. The only non-obvious fact is that it is closed under multiplication, and this follows from the property $V\left(w_{a}\right) V\left(w_{b}\right)=p V\left(w_{c}\right)$.

We now define a map

as follows. Fix a presentation

$$
\varphi: \Omega_{\widetilde{B}^{\dagger} / W(k)} \rightarrow D^{\prime}(C)
$$

$$
\widetilde{B}^{\dagger}=W(k)\left\langle x_{1}, \ldots, x_{n}, \frac{1}{g}\right\rangle^{\dagger}[z] /(P(z)) .
$$

Our map will send

$$
\varphi: x_{i} \mapsto \tilde{\phi}_{1}\left(x_{i}\right)+U\left(\tilde{\phi}_{2}\left(x_{i}\right)-\tilde{\phi}_{1}\left(x_{i}\right)\right)
$$

Because we have for $a, b \in D^{\prime}(C)^{0}, \gamma_{\varepsilon}(a b) \geq \gamma_{\varepsilon}(a)+\gamma_{\varepsilon}(b)$ and $\gamma_{\varepsilon}(a+b) \geq \min \left(\gamma_{\varepsilon}(a), \gamma_{\varepsilon}(b)\right)$, the proof of Proposition 2.28 in [5] can be mimicked to show that $D^{\prime}(C)^{0}$ is weakly complete. This immediately shows that $\varphi$ extends to $W(k)\left\langle x_{1}, \ldots, x_{n}\right\rangle^{\dagger}$.

As $g \in W(k)\left\langle x_{1}, \ldots, x_{n}\right\rangle^{\dagger}$, we have just shown $\varphi(g) \in D^{\prime}(C)$, and we must show this element is invertible. Write $\varphi(g)=\tilde{\phi}_{1}(g)+U f$, some $f$ such that $U f \in D^{\prime}(C)$. Because $\tilde{\phi}_{1}(g)$ is invertible in $W^{\dagger}(C)$,

$$
\frac{1}{\varphi(g)}=\frac{\tilde{\phi}_{1}(g)^{-1}}{1-U\left(-\tilde{\phi}_{1}(g)^{-1} f\right)},
$$

so to show $\varphi(g)$ is invertible it suffices to show that any $1-U \tilde{g} \in D^{\prime}(C)^{0}$ is invertible. Write

$$
\tilde{g}=V w_{0}+U p V w_{1}+U^{2} p^{2} V w_{2}+\cdots .
$$

It follows by a simple induction on $k$, starting with the base case $k=1$, that

$$
\tilde{g}^{k}=\sum_{i=0}^{\infty} U^{i} p^{k+i-1} V w_{i},
$$

$4{ }^{\text {e }}$ SÉRIE - TOME $44-2011-\mathrm{N}^{\circ} 2$ 
with $\gamma_{\varepsilon}\left(p^{k+i-1} V w_{i}\right) \geq 0$, same $\varepsilon$ as above. Hence

$$
1+U \tilde{g}+U^{2} \tilde{g}^{2}+\cdots \in D^{\prime}(C),
$$

as required.

Next we prove that $\varphi$ extends to $z$.

Lemma 3.29. - There exists

$$
\sum_{i=0}^{\infty} U^{i} c_{i} \in D^{\prime}(C)
$$

which is a root of $\varphi(P)(z)=z^{r}+\varphi\left(f_{1}\right) z^{r-1}+\cdots+\varphi\left(f_{r}\right)$.

Proof. - Because $D^{\prime}(C)$ is weakly complete (with respect to $(p)$ ), by Hensel's Lemma (Proposition 2.30 in [5]) it suffices to find a root modulo $p$. Because the ideal $\left(U^{2}\right) \subseteq(p)$, it will suffice for us to find a root modulo $U^{2}$. Thus we need only find the terms $c_{0}$ and $c_{1}$. As usual, $c_{0}=\tilde{\phi}_{1}(z)$. For $c_{1}$, we simply set $z=\sum_{i=0}^{\infty} U^{i} c_{i}$ in $\varphi(P)(z)=z^{r}+\varphi\left(f_{1}\right) z^{r-1}+$ $\cdots+\varphi\left(f_{r}\right)=0$ and check that this forces

$$
c_{1}=-\left(\tilde{\phi}_{1}(P)^{\prime}(z)\right)^{-1}\left(\left(\tilde{\phi}_{2}\left(f_{1}\right)-\tilde{\phi}_{1}\left(f_{1}\right)\right) c_{0}^{r-1}+\cdots+\tilde{\phi}_{2}\left(f_{r}\right)-\tilde{\phi}_{1}\left(f_{r}\right)\right) .
$$

We have now shown the existence of a map $\varphi: \widetilde{B}^{\dagger} \rightarrow D^{\prime}(C)^{0}$. We extend it to a map, also denoted by $\varphi$, of complexes,

$$
\varphi: \Omega_{\widetilde{B}^{\dagger} / W(k)} \rightarrow D^{\prime}(C)
$$

The chain homotopy promised in our proposition will factor through its image. This motivates the following.

Definition 3.30. - Let $D(C) \subseteq D^{\prime}(C)$ denote the image of $\varphi$.

We give now a more explicit description of what terms in $D(C)$ look like.

Lemma 3.31. - (i) Let $x$ denote some element of $\Omega_{\widetilde{B}^{\dagger} / W(k)}^{d}$. Write

$$
\varphi(x)=\cdots+U^{i+1} w^{\prime}+U^{i} d U w^{\prime \prime}+\cdots
$$

where $i \geq 0$. Then we may write $w^{\prime}=p^{\max (i-d, 0)} \mu_{i}$ and $w^{\prime \prime}=p^{\max (i-d+1,0)} \eta_{i}$ with $\mu_{i}, \eta_{i} \in \mathrm{Fil}^{1} W^{\dagger} \Omega_{C / k}$.

(ii) We may find $\varepsilon, G$ depending only on $x$ such that $\gamma_{\varepsilon}(w) \geq G$ for each coefficient $w$.

Proof. - (i) We prove this by induction on $d$. The base case $d=0$ has already been shown. Inductively assume the result for $x$ of degree $d-1$.

A term $x$ in degree $d$ may be written as a finite sum of terms $b d x_{i_{1}} \cdots d x_{i_{d}}$ with $b \in \widetilde{B}^{\dagger}$ and $x_{i_{j}}$ one of the generators of the polynomial algebra of which we have taken an étale extension. We will show the result for $b d x_{1} \cdots d x_{d}$. Extending to other index sets is trivial, and extending to finite sums is easy.

We are assuming the result for $\varphi\left(b d x_{1} \cdots d x_{d-1}\right)$, which is possibly just $\varphi(b)$. And we know

$$
\varphi\left(d x_{d}\right)=d \tilde{\phi}_{1}\left(x_{d}\right)+d U V\left(w_{d}\right)+U d V\left(w_{d}\right) .
$$

The result concerning the form of the coefficients now follows easily.

(ii) We again may restrict to the case of a term $b d x_{i_{1}} \cdots d x_{i_{d}}$. Concerning $\varphi(b)$, we already know the result. There are only finitely many nonzero terms of the form $d x_{i_{1}} \cdots d x_{i_{d}}$ 
(varying $d$ allowed). Thus we can find $\varepsilon^{\prime}, G^{\prime}$ such that every coefficient $w$ appearing in some term $\varphi\left(d x_{i_{1}} \cdots d x_{i_{d}}\right)$ satisfies $\gamma_{\varepsilon^{\prime}}(w) \geq G^{\prime}$. The result now follows from the fact that there exist $\varepsilon^{\prime \prime}, G^{\prime \prime}$ such that for any $\gamma_{\varepsilon}(\eta) \geq G, \gamma_{\varepsilon^{\prime}}(w) \geq G^{\prime}$ we have $\gamma_{\varepsilon^{\prime \prime}}(\eta \wedge w) \geq G^{\prime \prime}$.

Let $h_{0}, h_{1}$ denote the maps of differential graded algebras $D^{\prime}(C) \rightarrow W^{\dagger} \Omega_{C / k}$ which send $U \mapsto 0$ and $U \mapsto 1$, respectively. Our definition of $D^{\prime}(C)^{0}$ immediately implies that the image in degree zero really does land in $W^{\dagger}(C)$, and hence the image lands there in every degree. We also let $h_{0}, h_{1}$ denote their restrictions to $D(C)$.

Clearly we have $h_{0} \circ \varphi=\tilde{\phi}_{1}$ and $h_{1} \circ \varphi=\tilde{\phi}_{2}$, because both sides agree in degree zero. We define $p^{\kappa} L: D(C)^{\bullet} \rightarrow W^{\dagger} \Omega_{C / k}^{\bullet-1}$ by setting

$$
p^{\kappa} L\left(U^{j} \omega_{j}\right)=0 \text { and } p^{\kappa} L\left(U^{j} d U \wedge \omega_{j}\right)=\frac{p^{\kappa} \omega_{j}}{j+1},
$$

and then extending to all of $D(C)$ in the obvious way. Of course, it is not at all clear that our map has image where we claim.

Lemma 3.32. - The map $p^{\kappa} L$ has image in $W^{\dagger} \Omega_{C / k}$.

Proof. - We first show it maps to $W \Omega_{C / k}$, and then establish overconvergence. For an arbitrary $x \in \Omega_{\widetilde{B}^{\dagger} / W(k)}$, write

$$
\varphi(x)=\cdots+U^{j} d U \wedge \omega_{j}+\cdots
$$

as in the previous lemma. From the lemma, it suffices that

$$
\kappa+\max (j-\operatorname{dim} B+1,0) \geq\left\lfloor\log _{p}(j+1)\right\rfloor .
$$

For the case $j-\operatorname{dim} B+1>0$, check the specific case $j=\operatorname{dim} B$, then note that the left hand side grows faster with $j$ than the right hand side. For the case $j \leq \operatorname{dim} B-1$, we want to prove $\left\lfloor\log _{p} \operatorname{dim} B\right\rfloor \geq\left\lfloor\log _{p}(j+1)\right\rfloor$, which in this case is obvious.

Now we must check overconvergence. We are done if we verify the existence of $\varepsilon^{\prime}, G^{\prime}$ independent of $j$ such that $\gamma_{\varepsilon^{\prime}}\left(\frac{p^{\kappa} \omega_{j}}{j+1}\right) \geq G^{\prime}$. For arbitrary $\omega_{j} \in W^{\dagger} \Omega_{C / k}$ with $\gamma_{\varepsilon}\left(\omega_{j}\right) \geq G$ this is not true. But as before we know that

$$
p^{m^{\prime}} \mid \frac{p^{\kappa} \omega_{j}}{j+1}, \text { where } m^{\prime} \geq j-\operatorname{dim} B+\kappa+1-\left\lfloor\log _{p}(j+1)\right\rfloor .
$$

There exists $N$ depending only on $\operatorname{dim} B$ such that for $j \geq N, m^{\prime} \geq\left\lfloor\log _{p}(j+1)\right\rfloor$. So the following claim applies to all but finitely many terms in $\varphi(x)$.

ClaIm. - Let $\omega_{j} \in W^{\dagger} \Omega_{C / k}$. If $p^{\left\lfloor\log _{p}(j+1)\right\rfloor} \mid \frac{p^{\kappa} \omega_{j}}{j+1}$ and $\gamma_{\varepsilon}\left(p^{\kappa} \omega_{j}\right) \geq G$, then there exist $\varepsilon^{\prime}, G^{\prime}$ depending only on $\varepsilon, G$ with $\gamma_{\varepsilon^{\prime}}\left(\frac{p^{\kappa} \omega_{j}}{j+1}\right) \geq G^{\prime}$.

Proof. - It suffices to prove this for the equivalent norm $\gamma^{\prime}$ of page 223. We shall prove the result for $\left(\varepsilon^{\prime}, G^{\prime}\right)=\left(\frac{\varepsilon}{2}, \frac{G}{2}\right)$. Let $l:=\log _{p}(j+1)$. Pick an $\eta$ such that $p^{2 l} \eta=p^{\kappa} \omega_{j}$. Write $C:=\gamma_{\varepsilon}^{\prime}(\eta)$. From Corollary 2.24 or rather its evident generalisation to finite étale extensions over $A_{f}$ we know $\gamma_{\varepsilon}^{\prime}\left(p^{2 l} \eta\right)=C+2 l$, so from our assumption $C+2 l \geq G$. We also have $\gamma_{\frac{\varepsilon}{2}}^{\prime}(\eta) \geq \frac{C}{2}$, and so

as claimed.

$$
\gamma_{\frac{\varepsilon}{2}}^{\prime}\left(p^{l} \eta\right) \geq \frac{C}{2}+l \geq \frac{G}{2}
$$

4 e SÉRIE - TOME $44-2011-$ No $^{\circ}$ 
This proves that for all but finitely many terms $a$ in $p^{\kappa} L(\varphi(x)), \gamma_{\frac{\varepsilon}{2}}(a) \geq \frac{G}{2}$. For the other terms $b$ in $p^{\kappa} L(\varphi(x))$, we know $\gamma_{\varepsilon}(b(j+1)) \geq G$, with $j+1 \leq N+1$. Thus we can find $\varepsilon^{\prime \prime}, G^{\prime \prime}$ with $\gamma_{\varepsilon^{\prime \prime}}(a) \geq G^{\prime \prime}$ and $\gamma_{\varepsilon^{\prime \prime}}(b) \geq G^{\prime \prime}$ for all $a, b$ as above, which covers everything. This completes the proof that $p^{\kappa} L(\varphi(x))$ is indeed overconvergent.

Now we are basically done. It is trivial to check that $p^{\kappa} L$ is a homotopy between $p^{\kappa} h_{0}$ and $p^{\kappa} h_{1}$. Thus $p^{\kappa} L \circ \varphi$ is a homotopy between $p^{\kappa} h_{0} \circ \varphi=\tilde{\phi}_{1}$ and $p^{\kappa} h_{1} \varphi=\tilde{\phi}_{2}$. For the convenience of the reader, we state explicitly the sign convention:

$$
d(\omega \wedge \eta)=d \omega \wedge \eta+(-1)^{i} \omega \wedge d \eta
$$

where $\omega$ is in degree $i$.

\section{Comparison with rigid cohomology}

Let $X=\operatorname{Spec} A$ be a smooth affine scheme over a perfect field $k$ of characteristic $p>0$. In this section we define a canonical morphism from the rigid cohomology of $X$ to the de Rham-Witt cohomology.

Let $W=W(k)$ be the ring of Witt vectors.

Definition 4.1. - A special frame is a pair $(X, F)$ such that $F=\operatorname{Spec} B$ is a smooth affine scheme over $W$ and $X=\operatorname{Spec} A$ is a smooth affine scheme over $k$ which is a closed subscheme of $F$. The comorphism of this embedding is an epimorphism $B \rightarrow A$. We will also say that $(A, B)$ is a special frame.

Assume moreover that we are given a homomorphism $\varkappa: B \rightarrow W(A)$ which lifts $B \rightarrow A$. Then we call $(X, F, \varkappa)$ a Witt frame. If the image of $\varkappa$ is contained in $W^{\dagger}(A)$ the Witt frame is called overconvergent.

Let $(X, F, \varkappa)$ be a Witt frame. We denote by $\hat{F}$ the formal scheme which is the completion of $F$ in the ideal sheaf generated by $p$. Let $] X[\hat{F}$ be the tubular neighborhood (Berthelot [1]) of $X$ in the rigid analytic space $\hat{F}_{K}$ associated to the formal scheme $\hat{F}$. We will construct a natural map

$$
\Gamma(] X\left[\hat{F}, \Omega_{X[\hat{F}}\right) \rightarrow W \Omega_{X / k} \otimes \mathbb{Q} .
$$

It is enough to define a map

$$
\Gamma(] X\left[_{\hat{F}}, \theta_{] X[\hat{F}}\right) \rightarrow W(A) \otimes \mathbb{Q} .
$$

From this we can deduce (4.2) by the universal property of Kähler differentials. Let $\hat{F}_{/ X}$ be the formal completion of $F$ along $X$. By [1] 1.1.4 (ii) the tubular neighborhood $] X\left[{ }_{\hat{F}}\right.$ coincides with the rigid analytic space associated with the formal scheme $\hat{F}_{/ X}$. Let $I$ be the kernel of the homomorphism $B \rightarrow A$. We denote by $R$ the completion of $B$ in the ideal $I$. We have $\hat{F}_{/ X}=\operatorname{Spf} R$. The associated rigid analytic space is defined as follows: We choose a set of generators $f_{1}, \ldots, f_{m}$ of $I$. For a natural number $n$ we denote by $R_{n}^{\wedge}$ the $p$-adic completion of

$$
R_{n}=R\left[T_{1}, \ldots, T_{m}\right] /\left(f_{1}^{n}-p T_{1}, \ldots, f_{m}^{n}-p T_{m}\right)
$$


Then $R_{n}^{\wedge} \otimes \mathbb{Q}$ is an affinoid algebra and we have by definiton

$$
\Gamma(] X\left[_{\hat{F}}, \theta_{] X{ }_{\hat{F}}}\right)=\lim _{\longleftarrow} R_{n}^{\wedge} \otimes \mathbb{Q} .
$$

To define (4.3) it suffices to define a compatible system of maps

$$
R_{n} \rightarrow W(A)
$$

for $n$ large enough. The homomorphism $\varkappa$ maps $I$ to $V W(A)$. Since $W(A)$ is complete in the ideal $V W(A)$ the homomorphism $\varkappa$ extends to a morphism

$$
R \rightarrow W(A) .
$$

Since $\varkappa\left(f_{i}\right) \in V W(A)$ for $i=1, \ldots, m$ we obtain for $n \geq 2$ :

$$
\varkappa\left(f_{i}^{n}\right) \in p^{n-1} V W(A) .
$$

Since $p$ is not a zero divisor in $W(A)$ the element $(1 / p) \varkappa\left(f_{i}^{n}\right) \in W(A)$ is well defined. Mapping $T_{i}$ to this element we obtain the desired compatible system of maps (4.4). This finishes the definition of (4.3).

This construction is clearly functorial in the following sense: Assume we have a second special frame $\left(X_{1}, F_{1}, \varkappa_{1}\right)$. We set $X_{1}=\operatorname{Spec} A_{1}$ and $F_{1}=\operatorname{Spec} B_{1}$. Assume that we are given a morphism of Witt frames

$$
(X, F, \varkappa) \rightarrow\left(X_{1}, F_{1}, \varkappa_{1}\right) .
$$

This induces a morphism of formal schemes $\hat{F}_{/ X} \rightarrow \hat{F}_{1 / X_{1}}$ and therefore a morphism of the tubular neighborhoods $] X\left[{ }_{\hat{F}} \rightarrow\right] X_{1}\left[{ }_{\hat{F}}\right.$. Our construction gives a commutative diagram

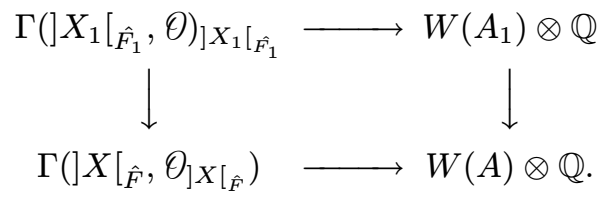

This also establishes the functoriality of the morphism (4.2).

Let $(X, F)$ be a special frame. We choose an embedding $F \subset \mathbb{A}_{W}^{n}$ in the affine space with comorphism

$$
W\left[X_{1}, \ldots, X_{n}\right] \rightarrow B .
$$

We write $E=\mathbb{A}_{W}^{n}$. Let $\mathbb{A}_{W}^{n} \subset P=\mathbb{P}_{W}^{n}$ be the canonical embedding

$$
X \rightarrow E \rightarrow P .
$$

We see easily that $] X\left[_{\hat{E}}=\right] X\left[_{\hat{P}}\right.$. We denote by $Q$ the closure of $F$ in $P$. Let $Y$ be the closure of $X$ in $P$. Let $\hat{Q}$ be the completion in the ideal $p$. Then

$$
X \rightarrow Y \rightarrow \hat{Q}
$$

is a frame in the sense of rigid cohomology. By this we mean that the embeddings $X \rightarrow Y$ and $Y \rightarrow \hat{Q}$ satisfy the assumptions for the definition of the rigid cohomology groups of $X$ in [2] 1.3 .

Our aim is to give an explicit description of a fundamental system of strict neighborhoods [1] (1.2.1) of $] X\left[_{\hat{Q}}=\right] X\left[_{\hat{F}}\right.$ in $] Y\left[_{\hat{Q}}\right.$.

Let us denote by $F_{K}^{\text {an }}$ the rigid analytic space associated to the scheme $F_{K}$. We have $F_{K}^{\mathrm{an}} \subset Q_{K}^{\mathrm{an}}=\hat{Q}_{K}$. It is clear that $\left.F_{K}^{\mathrm{an}} \cap\right] Y{ }_{\hat{Q}}$ is a strict neighborhood of $] X\left[_{\hat{F}}\right.$. We propose 
to give an intrinsic description of the strict neighborhoods which does not depend on the particular embedding $F \subset \mathbb{A}_{W}^{n}$.

It is enough to describe a fundamental system of strict neighborhoods of $] X[=] X{ }_{\hat{P}}$ in $] Y[=] Y\left[\hat{P}_{P}\right.$. The strict neighborhoods above are then obtained by intersecting with $\hat{Q}_{K} \subset \hat{P}_{K}$.

Let $X=\operatorname{Spec} k\left[X_{1}, \ldots, X_{n}\right] /\left(\bar{f}_{1}, \ldots, \bar{f}_{m}\right)$. Let $f_{j} \in W\left[X_{1}, \ldots, X_{n}\right]$ for $j=1, \ldots, m$ be liftings of the polynomials $\bar{f}_{j}$, such that $d_{j}=\operatorname{deg} \bar{f}_{j}=\operatorname{deg} f_{j}$. We take homogeneous coordinates $X_{i}=T_{i} / T_{0}$ for $i=1, \ldots, n$. Consider the homogeneous polynomials for $j=1, \ldots, m$ :

$$
F_{j}\left(T_{0}, \ldots, T_{n}\right)=T_{0}^{d_{j}} f_{j}\left(T_{1} / T_{0}, \ldots, T_{n} / T_{0}\right) .
$$

We denote by $\bar{F}_{j}$ the residue class modulo $p$. Then $Y \subset \mathbb{P}_{k}^{n}$ is given by the equations:

$$
\bar{F}_{j}\left(T_{0}, \ldots, T_{n}\right)=0 .
$$

We write a point $\left(t_{0}, \ldots, t_{n}\right)$ of $\hat{P}_{K}=P_{K}^{\text {an }}$ always in such a way that $\left|t_{i}\right| \leq 1$ for all $i=1, \ldots, n$ and such that we have equality for at least one index. The tubular neighborhood of $Y$ is:

$$
] Y\left[=\left\{\left(t_{0}, \ldots, t_{n}\right) \in \hat{P}_{K}|| F_{j}\left(t_{0}, \ldots, t_{n}\right) \mid<1\right\} .\right.
$$

For $\eta<1$ we write:

$$
] Y{ }_{\eta}=\left\{\left(t_{0}, \ldots, t_{n}\right) \in \hat{P}_{K}|| F_{j}\left(t_{0}, \ldots, t_{n}\right) \mid \leq \eta\right\} .
$$

Let $Z \subset Y$ denote the intersection of $Y$ with the hyperplane $\left\{T_{0}=0\right\}$. We have disjoint decompositions

$$
Y=X \sqcup Z, \quad] Y[=] X[\sqcup] Z[.
$$

We follow the notations of [1] 1.2. For $\lambda<1$ we have

$$
] Z\left[_{\lambda}=\right] Y\left[\cap\left\{\left|t_{0}\right|<\lambda\right\} .\right.
$$

Then $\left.U_{\lambda}^{\prime}=\right] Y[\backslash] Z\left[{ }_{\lambda}\right.$ is a strict neighborhood of $] X\left[\right.$. We set $\left.U_{\lambda, \eta}^{\prime}=\right] Y\left[{ }_{\eta} \cap U_{\lambda}^{\prime}\right.$. We have the inclusions

$$
\begin{aligned}
& U_{\lambda, \eta}^{\prime} \subset U_{\lambda^{\prime}, \eta}^{\prime}, \text { for } 1>\lambda>\lambda^{\prime}>0, \\
& U_{\lambda, \eta^{\prime}}^{\prime} \subset U_{\lambda, \eta}^{\prime}, \text { for } 1>\eta>\eta^{\prime}>0 .
\end{aligned}
$$

Let $\underline{\lambda}=\left\{\lambda_{i}\right\}$ and $\underline{\eta}=\left\{\eta_{i}\right\}$ be two monotonically increasing sequences of real numbers which converge to 1 . Then we set

$$
U_{\underline{\lambda}, \underline{\eta}}^{\prime}=\bigcup U_{\lambda_{i}, \eta_{i}}^{\prime}
$$

By [1] the sets $U_{\underline{\lambda}, \underline{\eta}}^{\prime}$ form a fundamental system of strict neighborhoods of $] X[$.

Let $E^{\text {an }} \subset \hat{P}_{K}=P^{\text {an }}=\left(\mathbb{P}_{K}^{n}\right)^{\text {an }}$ be the analytic variety associated to $\mathbb{A}_{K}^{n}$. We have $U_{\lambda}^{\prime} \subset E^{\text {an }}$. If $\mathbf{B}(0,1 / \lambda)$ denotes the closed ball of radius $1 / \lambda$ around 0 in $E^{\text {an }}$ we can write

$$
\left.U_{\lambda}^{\prime}=\right] Y\left[\cap \mathbf{B}(0,1 / \lambda), \quad U_{\lambda, \eta}^{\prime}=\right] Y\left[{ }_{\eta} \cap \mathbf{B}(0,1 / \lambda) .\right.
$$

We describe $] Y\left[\cap E^{\text {an }}\right.$ in affine coordinates. Consider a point $\left(t_{0}, \ldots, t_{n}\right) \in P_{K}^{\text {an }}$ with $t_{0} \neq 0$ and let $\left(x_{1}, \ldots, x_{n}\right)$ be the affine coordinates. We find:

$$
1 /\left|t_{0}\right|=\max \left\{1,\left|x_{1}\right|, \ldots,\left|x_{n}\right|\right\} .
$$


Therefore the defining inequalities for $] Y$ [ respectively $] Y{ }_{\eta}$ become

$$
\begin{aligned}
& \left|f_{j}\left(x_{1}, \ldots, x_{n}\right)\right|<\max \left\{1,\left|x_{1}\right|^{d_{j}}, \ldots,\left|x_{n}\right|^{d_{j}}\right\}, \\
& \left|f_{j}\left(x_{1}, \ldots, x_{n}\right)\right| \leq \eta \max \left\{1,\left|x_{1}\right|^{d_{j}}, \ldots,\left|x_{n}\right|^{d_{j}}\right\},
\end{aligned}
$$

for $j=1, \ldots, m$.

We set

$$
U_{\lambda}=\left\{\left(x_{1}, \ldots, x_{n}\right) \in \mathbf{B}(0,1 / \lambda)|| f_{j}\left(x_{1}, \ldots, x_{n}\right) \mid<1, \text { for } j=1, \ldots, m\right\} .
$$

We find $U_{\lambda} \subset U_{\lambda}^{\prime}$. We set $\left.U_{\lambda, \eta}=U_{\lambda} \cap\right] Y{ }_{\eta}$. These are affinoid subsets of $U_{\lambda, \eta}^{\prime}$ :

$$
U_{\lambda, \eta}=\left\{\left(x_{1}, \ldots, x_{n}\right) \in \mathbf{B}(0,1 / \lambda)|| f\left(x_{1}, \ldots, x_{n}\right) \mid \leq \eta\right\} .
$$

Lemma 4.12. - For each real $\eta<1$ there are reals $\lambda_{0}<1$ and $\eta_{0}<1$ such that

$$
U_{\lambda, \eta}^{\prime} \subset U_{\lambda, \eta^{\prime}}, \text { for } \lambda>\lambda_{0}, \eta^{\prime}>\eta_{0} .
$$

Proof. - We choose $\lambda_{0}$ in such a way that $|\eta|<\lambda_{0}^{d_{j}}$ for each index $d_{j}$. Then we find for $\lambda>\lambda_{0}$ and $\left|x_{i}\right| \leq 1 / \lambda$ that

$$
\eta\left|x_{i}\right|^{d_{j}} \leq \eta / \lambda^{d_{j}}<\eta / \lambda_{0}^{d_{j}}<\eta_{0}<1
$$

for a suitable $\eta_{0}$. This proves the assertion.

Lemma 4.13. - We define $U_{\underline{\lambda}, \underline{\eta}}$ for monotonic sequences $\underline{\lambda}$ and $\underline{\eta}$ by replacing $U^{\prime}$ by $U$ in (4.8). Then the $U_{\underline{\lambda}, \underline{\eta}}$ are a fundamental system of strict neighborhoods of $] X[$ in $] Y[$.

Proof. - Because of the inclusions $U_{\lambda_{i}, \eta_{i}} \subset U_{\lambda_{i}, \eta_{i}}^{\prime}$ it is enough to show that $U_{\underline{\lambda}, \underline{\eta}}$ is a strict neighborhood of $] X$. For each $i \in \mathbb{N}$ we set $\tilde{\eta}_{i}=\eta_{i}(1+(1 / i))^{-1}<\eta_{i}$. We choose $1>\tilde{\lambda}_{i}>\lambda_{i}$ such that $\tilde{\eta}_{i} / \tilde{\lambda}_{i}^{d_{j}}<\eta_{i}$ for each index $d_{j}$. The proof of the last lemma shows that

$$
U_{\tilde{\lambda}_{i}, \tilde{\eta}_{i}}^{\prime} \subset U_{\lambda_{i}, \eta_{i}} .
$$

Since $\tilde{\eta}_{i}<1$ and $\tilde{\lambda}_{i}<1$ are sequences which converge to 1 the set $U_{\underline{\tilde{\lambda}}, \tilde{\eta}}^{\prime}$ is a strict neighborhood of $] X\left[\right.$. The inclusion above shows that $U_{\underline{\lambda}, \underline{\eta}}$ is a strict neighborhood of $] X$.

Proposition 4.14. - Let $(X, F)$ be a special frame. Let $F \subset E=\mathbb{A}_{W}^{n}$ be an embedding in an affine space. Let $U_{\underline{\lambda}, \underline{\eta}} \subset E_{K}^{\text {an }}$ be defined by (4.11).

Let $X \rightarrow Y \rightarrow \hat{Q}$ be associated to the embedding $F \subset E(4.7)$. Then $V_{\underline{\lambda}, \underline{\eta}}=U_{\underline{\lambda}, \underline{\eta}} \cap F_{K}^{\text {an }}$ is a fundamental system of strict neighborhoods of $] X\left[_{\hat{Q}}\right.$ in $] Y\left[{ }_{\hat{Q}}\right.$.

Proof. - We just proved this in the case where $F=E$ is an affine space and $P=Q$ is the projective space. In general one obtains the strict neighborhoods of $] X\left[_{\hat{Q}}\right.$ in $] Y$ [ ${ }_{\hat{Q}}$ by intersecting with the strict neighborhoods of $] X{ }_{\hat{P}}$ in $] Y\left[{ }_{\hat{P}}\right.$. This proves the proposition.

It is easy to see that we end up with a cofinal system of neighborhoods if we replace in the definition of the $U_{\lambda, \eta}$ the polynomials $f_{j}$ by $f_{j}+p h_{j}$, where $h_{j} \in W\left[X_{1}, \ldots, X_{n}\right]$ are arbitrary polynomials. In other words, we may take for $f_{j}$ arbitrary liftings of $\bar{f}_{j}$ and drop the condition that $\operatorname{deg} f_{j}=\operatorname{deg} \bar{f}_{j}$. 
Corollary 4.15. - With the notations of the proposition let $F \subset \tilde{E}=\mathbb{A}_{W}^{l}$ be a second embedding which gives rise to a second frame $X \rightarrow \tilde{Y} \rightarrow \tilde{Q}$. Then the two systems of neighborhoods $V_{\underline{\lambda}, \underline{\eta}}$ and $\tilde{V}_{\underline{\lambda}, \underline{\eta}}$ of $] X\left[_{\hat{F}}\right.$ in $F_{K}^{\text {an }}$ are cofinal.

Proof. - We begin with a special case. Assume we are given a closed immersion $E \rightarrow \tilde{E}$ whose comorphism is of the form

$$
W\left[X_{1}, \ldots X_{n}, Z\right] \rightarrow W\left[X_{1}, \ldots X_{n}\right]
$$

where $Z$ is mapped to a polynomial $g\left(X_{1}, \ldots, X_{n}\right)$. Moreover we assume that the embedding $F \rightarrow \tilde{E}$ is the composite $F \rightarrow E \rightarrow \tilde{E}$.

We consider the morphism of frames in the sense of rigid cohomology

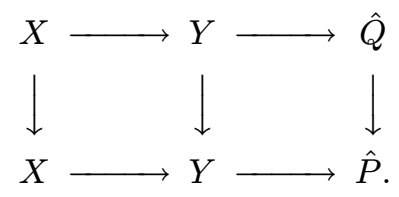

We obtain a fundamental system of strict neighborhoods of $] X\left[\hat{F}_{\hat{F}}\right.$ in $F_{K}^{\text {an }}$ by intersecting a fundamental system of strict neighborhoods of $] X\left[_{\hat{E}}\right.$ in $E_{K}^{\text {an }}$ with $F_{K}^{\text {an }}$. A similar remark applies for $\tilde{E}$.

We will now compare strict neighborhoods with respect to the frames

$$
X \rightarrow Y \rightarrow \hat{P}
$$

and

$$
X \rightarrow \tilde{Y} \rightarrow \hat{\tilde{P}}
$$

Let $f_{1}, \ldots, f_{m} \in W\left[X_{1}, \ldots, X_{n}\right]$ be polynomials whose reductions modulo $p$ define the closed subscheme $X \subset \mathbb{A}_{k}^{n}=E_{k}$.

For positive real numbers $\lambda, \eta<1$ we have considered the affinoid subsets:

$$
U_{\lambda, \eta} \subset \mathbf{B}(0,1 / \lambda) \subset E_{K}^{\text {an }},
$$

which are given by the inequalities

$$
\left|f_{j}\left(x_{1}, \ldots, x_{n}\right)\right| \leq \eta, \text { for } j=1, \ldots, m .
$$

Next we consider strict neighborhoods $\tilde{V} \subset \tilde{E}_{K}^{\text {an }}$ with respect to (4.18). We will show that $\tilde{V} \cap E_{K}^{\text {an }}$ is a strict neighborhood of $] X\left[_{\hat{E}}\right.$ with respect to (4.17). Moreover for each strict neighborhood $V$ of $] X\left[_{\hat{E}}\right.$ there is a strict neighborhood $\tilde{V}$ of $] X\left[_{\hat{E}}\right.$ such that $\tilde{V} \cap E_{K}^{\text {an }} \subset V$. By the remark after (4.16) this would imply that the strict neighborhoods of $] X{\left[\hat{F}_{K}\right.}_{E_{K}}$ in $F_{K}^{\text {an }}$ are the same with respect to the frames $X \rightarrow Y \rightarrow \hat{Q}$ and $X \rightarrow \tilde{Y} \rightarrow \hat{\tilde{Q}}$. This would prove the proposition in the special case above.

Let us consider the open sets (4.19) for the frame (4.18):

$$
\tilde{U}_{\lambda, \eta}=\mathbf{B}(0,1 / \lambda) \subset \tilde{E}_{K}^{\text {an }}
$$

They are given by the following inequalities

$$
\begin{aligned}
\left|f_{j}\left(x_{1}, \ldots, x_{n}\right)\right| & \leq \eta, \\
\left|z-g\left(x_{1}, \ldots, x_{n}\right)\right| & \leq \eta .
\end{aligned}
$$


This shows immediately that

$$
U_{\lambda, \eta} \supset \tilde{U}_{\lambda, \eta} \cap E_{K}^{\mathrm{an}}
$$

Therefore for each strict neighborhood $V=U_{\underline{\lambda}}, \underline{\eta}$ we have found the strict neighborhood $\tilde{V}=\tilde{U}_{\underline{\lambda}, \eta}$ such that $\tilde{V} \cap E_{K}^{\text {an }} \subset V$. We have to show that $\tilde{V} \cap E_{K}^{\text {an }}$ is a strict neighborhood. Let $t$ be the total degree of the polynomial $g$. Let $\rho>1$ be some real number. If $\left|x_{1}\right| \leq \rho, \ldots,\left|x_{n}\right| \leq \rho$ then we have

$$
\left|g\left(x_{1}, \ldots, x_{n}\right)\right| \leq \rho^{t} .
$$

This shows that

$$
U_{\lambda^{\frac{1}{t}, \eta}} \subset \tilde{U}_{\lambda, \eta}
$$

We see that $\tilde{V} \cap E_{K}^{\text {an }}$ is a strict neighborhood. This proves the proposition for the special case we started with.

Now we consider an arbitrary second closed immersion $F \rightarrow \mathbb{A}^{l}$. We obtain a diagonal embedding $F \rightarrow \mathbb{A}^{n} \times_{\text {Spec } W} \mathbb{A}^{l}$. We take coordinates $Y_{1}, \ldots, Y_{l}$ on $\mathbb{A}^{l}$. We compare the comorphisms of the diagonal embedding with the comorphism of $F \rightarrow \mathbb{A}^{n}$ :

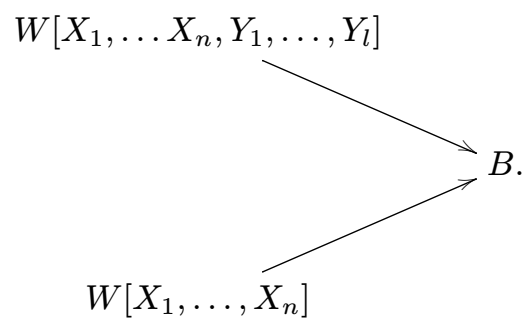

We find an epimorphism $W\left[X_{1}, \ldots X_{n}, Y_{1}, \ldots, Y_{l}\right] \rightarrow W\left[X_{1}, \ldots, X_{n}\right]$, which maps $X_{i}$ to $X_{i}$ which makes this diagram commutative. We obtain a diagram

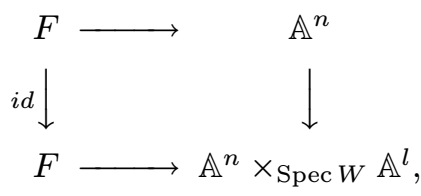

where the vertical arrow on the right hand side is the closed immersion defined above. But then the independence of strict neighborhoods in $F_{K}^{\text {an }}$ follows by induction from the case done above.

As a second corollary we prove the functoriality of strict neighborhoods.

Corollary 4.21. - Let $\left(X_{1}, F_{1}\right) \rightarrow\left(X_{2}, F_{2}\right)$ be a morphism of special frames. Let $V_{2} \subset F_{2, K}^{\text {an }}$ be a strict neighborhood of $] X_{2}\left[\hat{F}_{2}\right.$. Then the inverse image of $V_{2}$ by the map $F_{1, K}^{\mathrm{an}} \rightarrow F_{2, K}^{\mathrm{an}}$ contains a strict neighborhood of $] X_{1}\left[\hat{F}_{1}\right.$ in $F_{1, K}^{\mathrm{an}}$.

4 e SÉRIE - TOME $44-2011-\mathrm{N}^{\circ} 2$ 
Proof. - We may restrict to the case where the morphism of frames is of the following type:

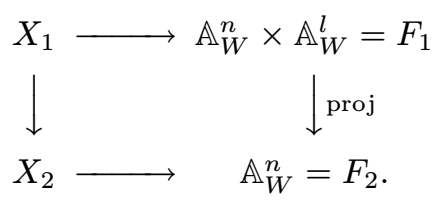

Consider the corresponding comorphisms

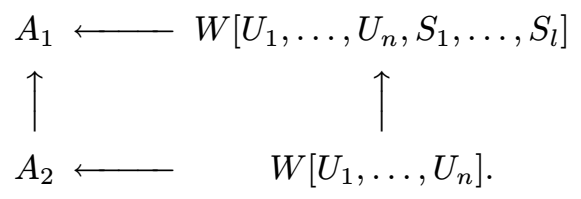

We choose polynomials $f_{1}, \ldots, f_{m} \in W\left[U_{1}, \ldots, U_{n}\right]$ whose reductions modulo $p$ generate the kernel of $k\left[U_{1}, \ldots, U_{n}\right] \rightarrow A_{2}$. Then we choose $g_{1}, \ldots, g_{k} \in W\left[U_{1}, \ldots, U_{n}, S_{1}, \ldots, S_{l}\right]$ such that the reductions of $f_{1}, \ldots, f_{m}, g_{1}, \ldots, g_{k}$ modulo $p$ generate the kernel of $k\left[U_{1}, \ldots, U_{n}, S_{1}, \ldots, S_{l}\right] \rightarrow A_{1}$. Then $U_{1, \lambda, \eta} \subset \mathbf{B}^{n+l}(1 / \lambda)$ is the subset of this closed ball given by the inequalities $\left|f_{i}\right| \leq \eta$ and $g_{j} \leq \eta$ for $j=1, \ldots m$ and $i=1, \ldots, k$. From this we conclude immediately that

$$
\operatorname{proj}\left(U_{1, \lambda, \eta}\right) \subset U_{2, \lambda, \eta},
$$

where proj : $\left(\mathbb{A}_{K}^{n}\right)^{\text {an }} \times\left(\mathbb{A}_{K}^{l}\right)^{\text {an }} \rightarrow\left(\mathbb{A}_{K}^{n}\right)^{\text {an }}$ is the projection. This proves the functoriality.

Let $(X, F, \varkappa)$ be an overconvergent Witt frame. Let $V \subset F_{K}^{\text {an }}$ be a strict neighborhood of ]$X\left[{ }_{\hat{F}}\right.$. For a sheaf of abelian groups $\mathcal{F}$ on $V$ Berthelot defines $j^{\dagger} \mathcal{F}$. If $W \subset V$ is an open and quasicompact subset

$$
\Gamma\left(W, j^{\dagger} \mathcal{F}\right)=\lim _{V^{\prime} \subset V} \Gamma\left(V^{\prime} \cap W, \mathcal{F}\right)
$$

The rigid cohomology of $X$ is by definition

$$
R \Gamma_{\text {rig }}(X)=\mathbf{R} \Gamma\left(V, j^{\dagger} \Omega_{V}\right) .
$$

In particular this is independent of the chosen $V$ ([2] (1.2.5)).

We will now define a map

$$
\Gamma\left(V, j^{\dagger} \Omega_{V}\right) \rightarrow W^{\dagger} \Omega_{X / k} \otimes \mathbb{Q}
$$

This will be compatible with the morphism (4.2)

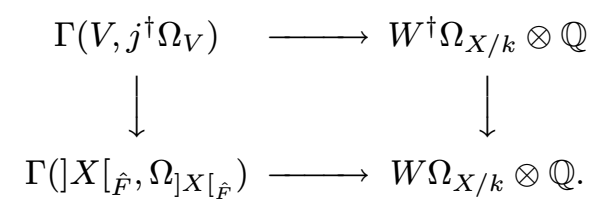

We begin with the case where $F=E$ is the affine space. We use on $W$ the $p$-adic absolute value, such that $|p|=1 / p$. For $\eta=p^{-1 / r}$ the affinoid algebra of $U_{\lambda, \eta}$ is

$$
\mathcal{T}=K\left\langle\lambda X_{1}, \ldots, \lambda X_{n}, T_{1}, \ldots T_{m}\right\rangle /\left(f_{1}^{r}-p T_{1}, \ldots, f_{m}^{r}-p T_{m}\right) .
$$

It consists of all power series

$$
\mathfrak{p}=\sum a_{I, J} \underline{X}^{I} \underline{T}^{J}, \quad a_{I, J} \in K,
$$


such that $\lim _{|I|+|J| \rightarrow \infty}\left|a_{I, J}\right|(1 / \lambda)^{|I|}=0$. We have seen that there is a homomorphism $\mathcal{J} \rightarrow W(A) \otimes \mathbb{Q}$ for $r \geq 2$. It maps the $X_{i}$ to $\xi_{i} \in W(A)$. Clearly we have $f_{j}\left(\xi_{1}, \ldots, \xi_{n}\right) \in$ $V W(A)$. We set

$$
f_{j}\left(\xi_{1}, \ldots, \xi_{n}\right)={ }^{V} \rho_{j}, \quad \text { for } j=1, \ldots, m .
$$

For $r \geq 3$ the variable $T_{j}$ is mapped to

$$
\left({ }^{V} \rho_{j}\right)^{r} / p=p^{r-2 V}\left(\rho_{j}^{r}\right) .
$$

Then the power series $\mathfrak{p}$ is mapped to

$$
\sum a_{I, J} p^{(r-2)|J|} \underline{\eta}^{I}\left({ }^{V}\left(\underline{\rho}^{r}\right)\right)^{J} .
$$

We have to show that this power series converges to an element in $W^{\dagger}(A) \otimes \mathbb{Q}$. Almost all coefficients $a_{I, J}$ are in $W$. Therefore we may assume that all these coefficients are in $W$. Since $W^{\dagger}(A)$ is a weakly complete $W$-algebra we see immediately that the series (4.23) represents an element of $W^{\dagger}(A)$.

Altogether we find a homomorphism

$$
\Gamma\left(U_{\lambda, \eta}, \theta_{U_{\lambda, \eta}}\right) \rightarrow W^{\dagger}(A) \otimes \mathbb{Q},
$$

which exists for each $\lambda$ and each $\eta$ with $\eta \geq p^{-1 / 3}$.

Let $V$ be a strict neighborhood of $] X$ [. It contains some $U_{\lambda, \eta}$ with $\eta \geq p^{-1 / 3}$. We have the morphism

$$
\Gamma\left(V, j^{\dagger} \theta_{V}\right) \rightarrow \lim _{V^{\prime} \subset V} \Gamma\left(V^{\prime} \cap U_{\lambda, \eta}, \theta_{V^{\prime} \cap U_{\lambda, \eta}}\right) .
$$

For each $V^{\prime}$ we find $\lambda^{\prime}>\lambda$ and $\eta^{\prime}>\eta$ such that $U_{\lambda^{\prime}, \eta^{\prime}} \subset V^{\prime}$. This implies $U_{\lambda^{\prime}, \eta} \subset V^{\prime} \cap U_{\lambda, \eta}$. This gives the canonical map

$$
\Gamma\left(V, j^{\dagger} \theta_{V}\right) \rightarrow \lim _{\overrightarrow{\lambda^{\prime}}} \Gamma\left(U_{\lambda^{\prime}, \eta}, \theta_{U_{\lambda^{\prime}, \eta}}\right) \rightarrow W^{\dagger}(A) \otimes \mathbb{Q} .
$$

By the universality of the de Rham complex we obtain a map

$$
\Gamma\left(V, j^{\dagger} \Omega_{V}\right) \rightarrow W^{\dagger} \Omega_{A / k} \otimes \mathbb{Q}
$$

where $V$ is any strict neighborhood of $] X[\hat{E}$.

Now we consider the case of a general overconvergent Witt frame $(X, F, \varkappa)$. We choose a closed embedding $F \subset E$ in an affine space $E$. Let

$$
W\left[X_{1}, \ldots, X_{n}\right] \rightarrow B
$$

be the corresponding comorphism as above. We obtain a commutative diagram

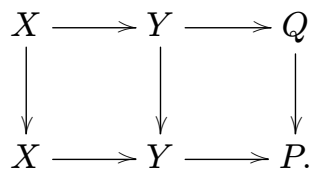

We have a closed immersion

$$
] Y\left[{ }_{\hat{Q}}=\hat{Q}_{K} \cap\right] Y[\hat{P} \rightarrow] Y[\hat{P}
$$

Let $U_{\lambda, \eta} \subset E_{K}^{\text {an }}$ as above. Then $V_{\lambda, \eta}=U_{\lambda, \eta} \cap F_{K}^{\text {an }}$ are exactly the neighborhoods " $U_{\lambda, \eta}$ " with respect to the frame $X \rightarrow Y \rightarrow Q$. The closed immersion of affinoids

$$
V_{\lambda, \eta} \rightarrow U_{\lambda, \eta}
$$

4 e SÉRIE - TOME $44-2011-$ No $^{\circ}$ 
is defined by the polynomials in the kernel of(4.26). Therefore we obtain an epimorphism

$$
\Gamma\left(U_{\lambda, \eta}, \theta_{U_{\lambda, \eta}}\right) \rightarrow \Gamma\left(V_{\lambda, \eta}, \theta_{V_{\lambda, \eta}}\right)
$$

whose kernel is generated by the elements in the kernel of (4.26). This shows that the morphism

$$
\Gamma\left(U_{\lambda, \eta}, \theta_{U_{\lambda, \eta}}\right) \rightarrow W^{\dagger}(A) \otimes \mathbb{Q}
$$

factors through a morphism

$$
\Gamma\left(V_{\lambda, \eta}, \vartheta_{V_{\lambda, \eta}}\right) \rightarrow W^{\dagger}(A) \otimes \mathbb{Q} .
$$

We conclude as above that for each strict neighborhood $V$ of $] X\left[_{\hat{F}}\right.$ we obtain a morphism

$$
\Gamma\left(V, j^{\dagger} \vartheta_{V}\right) \rightarrow W^{\dagger}(A) \otimes \mathbb{Q}
$$

and a comparison morphism

$$
\Gamma\left(V, j^{\dagger} \Omega_{V}\right) \rightarrow W^{\dagger} \Omega_{A / k} \otimes \mathbb{Q} .
$$

We will now show that the last morphism factors canonically through a morphism

$$
\mathbf{R} \Gamma\left(V, j^{\dagger} \Omega_{V}\right) \rightarrow W^{\dagger} \Omega_{A / k} \otimes \mathbb{Q} .
$$

Let $V$ be a fixed strict neighborhood of $] X\left[_{\hat{F}}\right.$ in $F_{K}^{\text {an }}$ as above. We begin with the natural restriction map

$$
\mathbf{R} \Gamma\left(V, j^{\dagger} \Omega_{V}\right) \rightarrow \mathbf{R} \Gamma\left(V_{\lambda, \eta}, j^{\dagger} \Omega_{V_{\lambda, \eta}}\right) .
$$

Let $V^{\prime} \subset V$ be a strict neighborhood. We write $\alpha_{V^{\prime}}: V^{\prime} \cap V_{\lambda, \eta} \rightarrow V_{\lambda, \eta}$ for the canonical immersion. By definition we have an isomorphism

$$
j^{\dagger} \Omega_{V_{\lambda, \eta}} \cong \lim _{\overrightarrow{V^{\prime}}} \alpha_{V^{\prime} *} \Omega_{V^{\prime} \cap V_{\lambda, \eta}} .
$$

Because $V_{\lambda, \eta}$ is quasicompact the inductive limit commutes with cohomology. We obtain a map:

$$
\mathbf{R} \Gamma\left(V, j^{\dagger} \Omega_{V}\right) \rightarrow \lim _{\overrightarrow{V^{\prime}}} \mathbf{R} \Gamma\left(V_{\lambda, \eta}, \alpha_{V^{\prime *}} \Omega_{V^{\prime} \cap V_{\lambda, \eta}}\right) .
$$

Again for each $V^{\prime}$ we find $\lambda^{\prime}$ such that $V_{\lambda^{\prime}, \eta} \subset V^{\prime} \cap V_{\lambda, \eta}$. The restriction to the affinoids $V_{\lambda^{\prime}, \eta}$ finally gives a map

$$
\mathbf{R} \Gamma\left(V, j^{\dagger} \Omega_{V}\right) \rightarrow \lim _{\overrightarrow{\lambda^{\prime}}} \mathbf{R} \Gamma\left(V_{\lambda^{\prime}, \eta}, \Omega_{V_{\lambda^{\prime}, \eta}}\right) \cong \lim _{\overrightarrow{\lambda^{\prime}}} \Gamma\left(V_{\lambda^{\prime}, \eta}, \Omega_{V_{\lambda^{\prime}, \eta}}\right) \rightarrow W^{\dagger} \Omega_{A / k} \otimes \mathbb{Q} .
$$

This completes the definition of the morphism (4.29). Taking into account (4.22) we obtain for each overconvergent Witt frame $(X, F, \varkappa)$ a morphism

$$
R \Gamma_{\text {rig }}(X) \rightarrow W^{\dagger} \Omega_{A / k} \otimes \mathbb{Q}
$$

This morphism is functorial in the triple $(X, F, \varkappa)$. We note that in the case where $F$ lifts $X$, i.e. $X \cong F \times{ }_{\text {Spec } W}$ Spec $k$, the complex $\Gamma\left(V, j^{\dagger} \Omega_{V}\right) \cong \mathbf{R} \Gamma\left(V, j^{\dagger} \Omega_{V}\right)$ is by [2] (1.10 Proposition) quasi-isomorphic to the Monsky-Washnitzer complex associated to the weak completion of $B$.

Proposition 4.31. - The comparison morphism (4.29) for overconvergent Witt frames is an isomorphism in the derived category. The induced isomorphism (4.30) is independent of the overconvergent Witt frame we have chosen. 
Proof. - We begin to show the independence of (4.30). Let $\left(A, B^{\prime}, \kappa^{\prime}\right)$ be a second overconvergent Witt frame. We set $F^{\prime}=\operatorname{Spec} B^{\prime}$ and $B^{\prime \prime}=B \otimes_{W(k)} B^{\prime}$ and $F^{\prime \prime}=\operatorname{Spec} B^{\prime \prime}=F \times F^{\prime}$. We obtain an overconvergent Witt frame $B^{\prime \prime} \rightarrow W^{\dagger}(A)$ by taking the product of the overconvergent Witt lifts for $B$ and $B^{\prime}$. We consider the two projections

$$
F \longleftarrow F^{\prime \prime} \longrightarrow F^{\prime} \text {. }
$$

We may choose strict neighborhood $V \subset F_{K}^{\text {an }}, V^{\prime} \subset F_{K}^{\prime \text { an }}, V^{\prime \prime} \subset F_{K}^{\prime \prime \text { an }}$ such that $V^{\prime \prime}$ is mapped to $V$ respectively $V^{\prime}$ by the two projections. By the functoriality (4.29) this induces a commutative diagram

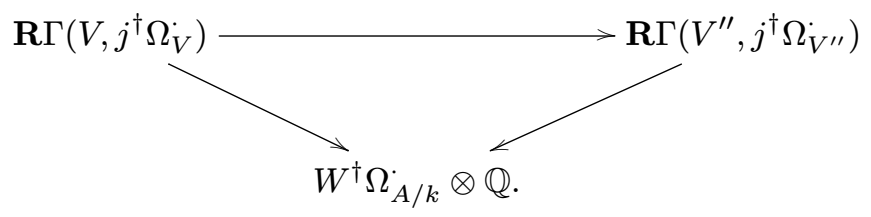

This shows that the comparison morphisms (4.30) for the overconvergent Witt frames $F$ and $F^{\prime \prime}$ are the same. Since the same is true for $F^{\prime}$ we have shown the independence.

By Proposition 3.24 there are overconvergent frames $(A, \tilde{A}, \psi)$ such that the associated morphism

$$
R^{i} \Gamma_{\text {rig }}(X) \cong H^{i}\left(\Omega_{\tilde{A}^{\dagger} / W} \otimes \mathbb{Q}\right) \rightarrow H^{i}\left(W^{\dagger} \Omega_{A / k}\right) \otimes \mathbb{Q}
$$

is an isomorphism for each $i \geq 0$. Therefore (4.29) is an isomorphism for arbitrarily chosen overconvergent frames.

To globalize our results we use dagger spaces [6]. We associate a dagger space to a special frame $(X, F)$. We choose an embedding $F \subset E=\mathbb{A}_{W}^{n}$. We begin to describe the dagger space structure on $] X[\hat{E}$.

We have

$$
] X\left[_{\hat{E}}=\left\{\left(x_{1}, \ldots, x_{m}\right) \in \mathbf{B}(0,1)|| f_{i}\left(x_{1}, \ldots, x_{n}\right) \mid<1\right\},\right.
$$

with the notations introduced after (4.7). We choose a natural number $u$ and we set $\eta_{u}=p^{-1 / u}$. Then $] X\left[_{\hat{E}}\right.$ is covered by the affinoids

$$
H_{\eta_{u}}=\left\{\left(x_{1}, \ldots, x_{m}\right) \in \mathbf{B}(0,1)|| f_{i}\left(x_{1}, \ldots, x_{n}\right) \mid \leq \eta_{u}\right\} .
$$

The affinoid algebra of $H_{\eta_{u}}$ is

$$
C_{\eta_{u}}=K<X_{1}, \ldots, X_{n}, S_{1}, \ldots, S_{m}>/\left(\ldots,\left(f_{i}^{u}-p S_{i}\right), \ldots\right),
$$

which over a suitable extension $\tilde{K}$ of $K$ becomes isomorphic to

$$
\tilde{K}\left\langle X_{1}, \ldots, X_{n}, T_{1}, \ldots, T_{m}\right\rangle /\left(\ldots,\left(f_{i}-p^{1 / u} T_{i}\right), \ldots\right) .
$$

We consider for $t>u$ the open immersion

$$
H_{\eta_{u}} \rightarrow U_{\lambda, \eta_{t}}
$$

(compare (4.11)). Over $\tilde{K}$ it is given by a comorphism

$$
\tilde{K}\left\langle\lambda X_{1}, \ldots, \lambda X_{n}, T_{1}^{\prime}, \ldots, T_{m}^{\prime}\right\rangle /\left(\ldots,\left(f_{i}-p^{1 / t} T_{i}^{\prime}\right), \ldots\right) \rightarrow C_{\eta_{u}}
$$

where $\lambda=p^{-1 / v}$ for an arbitrary chosen natural number $v$. The map sends the variables $\lambda X_{i}$ to $p^{1 / v} X_{i}$ and the variables $T_{i}^{\prime} \rightarrow p^{(1 / u)-(1 / t)} T_{i}$. This is an open immersion of $H_{\eta_{u}}$ to the interior of $U_{\lambda, \eta_{t}}$, i.e. $H_{\eta_{u}} \Subset U_{\lambda, \eta_{t}}$ in the sense of [3]. By [6] 2.21 this defines a dagger 
space structure on each $H_{\eta_{u}}$ and hence on $] X\left[_{\hat{E}}\right.$. We denote this dagger space by $] X\left[_{\hat{E}}^{\dagger}\right.$. Its completion is the rigid space $] X[\hat{E}$.

From the definition of the dagger space structure $H_{\eta_{u}}^{\dagger}$ we conclude that

$$
H^{0}\left(H_{\eta_{u}}^{\dagger}, \theta\right)=H^{0}\left(U_{\lambda, \eta_{t}}, j^{\dagger} \theta\right)
$$

We deduce an isomorphism for an arbitrary strict neighborhood $U \subset E^{\text {an }}$ of $] X\left[{ }_{\hat{E}}\right.$

$$
H^{0}(] X\left[_{\hat{E}}^{\dagger}, \theta\right)=H^{0}\left(U, j^{\dagger} \theta\right)
$$

(compare [6] §5).

Using the closed immersion $F_{K}^{\text {an }} \subset E_{K}^{\text {an }}$ we obtain also a dagger space structure on $] X\left[_{\hat{F}}\right.$. By definition this dagger structure depends only on the fundamental system of fundamental neighborhoods $V_{\underline{\lambda}, \underline{\eta}}$ given by Proposition 4.14. It follows that the dagger space $] X\left[_{\hat{F}}^{\dagger}\right.$ is functorial in $(X, F)$. If $U \subset F_{K}^{\text {an }}$ we obtain an isomorphism

$$
H^{0}(] X\left[_{\hat{F}}^{\dagger}, \theta\right)=H^{0}\left(U, j^{\dagger} \theta\right) \text {. }
$$

By [6] we have moreover that

$$
\mathbf{R} \Gamma(] X\left[_{\hat{F}}^{\dagger}, \Omega_{] X\left[_{\hat{F}}^{\dagger}\right.}\right)=R \Gamma_{\text {rig }}(X) .
$$

We associate to each special frame $(X, F)$ a specialization map. By [1] we have a morphism of ringed spaces

$$
] X\left[_{\hat{F}} \rightarrow \hat{F}_{/ X}\right.
$$

where the right hand side is the completion of $F$ in the closed subscheme $X$. If we view this as a morphism of Grothendieck topologies only we obtain a map

$$
\mathbf{s p}:] X\left[_{\hat{F}}^{\dagger}=\right] X[\hat{F} \rightarrow X
$$

(see [6] Thm. 2.19 for the last equality.)

We rewrite the comparison morphism as defined before (4.28) in terms of dagger spaces

$$
\Gamma(] X\left[_{\hat{F}}^{\dagger}, \Omega_{] X\left[_{\hat{F}}^{\dagger}\right.}\right) \rightarrow W^{\dagger} \Omega_{A / k} \otimes \mathbb{Q}
$$

where $X=\operatorname{Spec} A$.

We have also a local version of this morphism

$$
\mathbf{s p}_{*} \Omega_{] X\left[_{\hat{F}}^{\dagger}\right.} \rightarrow W^{\dagger} \Omega_{X / k} \otimes \mathbb{Q} \text {. }
$$

To see this we consider an open set $U=\operatorname{Spec} A_{\bar{f}} \subset X, \bar{f} \in A$. Let $f \in B$ be a lift of $\bar{f}$, where Spec $B=F$. The open set $] U\left[{ }_{\hat{F}} \subset\right] X\left[_{\hat{F}}\right.$ inherits the structure of a dagger space. To define (4.32) it is enough to show that this dagger space structure coincides with that given by the special frame $\left(U\right.$, Spec $\left.B_{f}\right)$. Indeed, form the commutative diagram

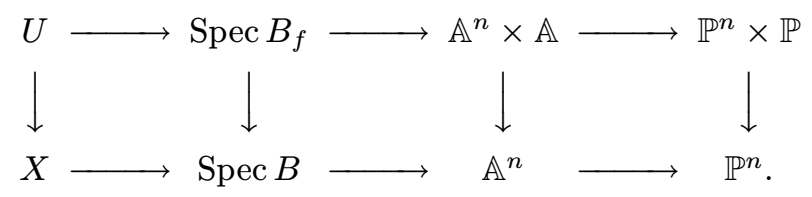


This induces a map of frames in the sense of rigid cohomology

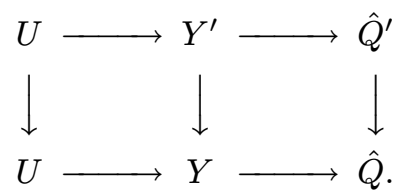

The last vertical arrow is proper and is an open immersion in a neighborhood of $U$. We conclude by [1], Thm. 1.3.5, that the strict tubular neighborhoods associated to the two frames are the same. This implies the desired isomorphism of dagger spaces.

Let now $X$ be a smooth quasiprojective scheme over $k$. Our next aim is the definition of a comparison morphism

$$
R \Gamma_{\text {rig }}(X) \rightarrow \mathbf{R} \Gamma\left(X, W^{\dagger} \Omega_{X / k}\right) \otimes \mathbb{Q} .
$$

Definition 4.33. - Let $R$ be a ring. We call $A$ a standard smooth algebra over $R$ if $A$ can be represented in the form

$$
A=R\left[X_{1}, \ldots X_{n}\right] /\left(f_{1}, \ldots f_{m}\right),
$$

where $m \leq n$ and the determinant

$$
\operatorname{det}\left(\frac{\partial f_{i}}{\partial X_{j}}\right), \quad i, j=1, \ldots m
$$

is a unit in $A$. We call $\mathrm{Spec} A$ a standard smooth scheme.

We remark that a localization of a standard smooth algebra by an element is again standard smooth. Since $X$ is smooth over $k$ it has a covering by standard smooth neighborhoods.

We choose an open embedding $X \rightarrow \operatorname{Proj} \phi$, where $\phi$ is a finitely generated graded algebra over $k$. We consider finite coverings $X=\cup_{i \in I} D^{+}\left(h_{i}\right)$, where the $h_{i} \in \&$ are homogeneous elements which have all the same degree. If we choose the covering sufficiently fine we may assume that all $X_{i}=D^{+}\left(h_{i}\right)$ are standard smooth schemes over $k$. For a subset $J=\left\{i_{1}, \ldots, i_{t}\right\} \subset I$ we set

$$
X_{J}=X_{i_{1}} \cap \cdots \cap X_{i_{t}} .
$$

We write $X_{J}=\operatorname{Spec} A_{J}$. Then $A_{J}$ is a localization of $A_{i_{1}}$ by a suitable element $\bar{g} \in A_{i_{1}}$.

Let $A$ as in Definition 4.33. We choose arbitrary liftings $\tilde{f}_{1}, \ldots, \tilde{f}_{m} \in W\left[X_{1}, \ldots, X_{n}\right]$. Let $B$ be a localization of $W\left[X_{1}, \ldots, X_{n}\right] /\left(\tilde{f}_{1}, \ldots, \tilde{f}_{m}\right)$ with respect to $\operatorname{det}\left(\frac{\partial \tilde{f}_{i}}{\partial X_{j}}\right)$, where $i, j=1, \ldots m$. Then $B$ is a standard smooth algebra which lifts $A$ over $W$.

We will choose for each $A_{i}$ a standard smooth lift $B_{i}$ as above. We set $F_{i}=\operatorname{Spec} B_{i}$ and obtain special frames $\left(X_{i}, F_{i}\right)$ for $i \in I$. For $J \subset I$ we consider the closed embedding

$$
X_{J} \rightarrow \prod_{i \in J} F_{i}
$$

This is a special frame.

4 e SÉRIE - TOME $44-2011$ - No 2 
Proposition 4.35. - Let us denote by $Q$ the dagger space which we introduced on the tubular neighborhood $] X_{J}\left[\right.$ with respect to the special frame (4.34) and let $\mathbf{s p ~ : Q} \rightarrow X_{J}$ be the specialization morphism. Then the canonical map

$$
\mathbf{s p}_{*} \Omega_{Q} \rightarrow \mathbf{R} \mathbf{s p}_{*} \Omega_{Q}
$$

is a quasiisomorphism.

Proof. - We will reduce this to a more special situation. The main ingredient is the strong fibration theorem of [1] 1.3.5. In terms of dagger spaces it has the following consequence.

Let $\left(Z, F_{1}\right)$ and $\left(Z, F_{2}\right)$ be special frames. We denote by $Q_{1}$ and $Q_{1}$ the corresponding dagger spaces. Let $\nu: F_{1} \rightarrow F_{2}$ be a morphism of frames which induces the identity on $Z$. If $\nu$ is étale in a neighborhood of $Z$ in $F_{1}$ then $\nu$ induces an isomorphism $Q_{1} \rightarrow Q_{2}$.

To see this we choose closed immersions $F_{1} \rightarrow \mathbb{A}_{W}^{m}$ and $F_{2} \rightarrow \mathbb{A}_{W}^{n}$. We consider the commutative diagram

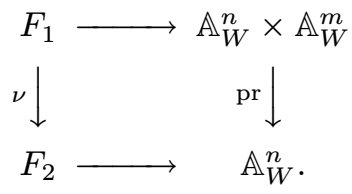

We denote by $P_{1}$ the closure of $F_{1}$ in $\mathbb{P}_{W}^{n} \times \mathbb{P}_{W}^{m}$ and by $P_{2}$ the closure of $F_{2}$ in $\mathbb{P}_{W}^{n}$. We note that $F_{1}$ is open in $P_{1}$ and $F_{2}$ is open in $P_{2}$. Let $Y_{1}$ resp. $Y_{2}$ be the closure of $Z$ in $P_{1}$ resp. $P_{2}$. Taking the $p$-adic completions we obtain a commutative diagram

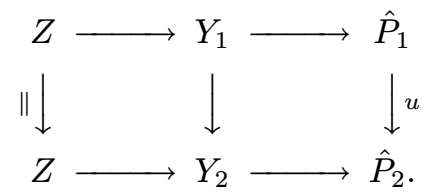

Then $u$ is proper and étale in a neighborhood of $Z$ in $\hat{P}_{1}$. Therefore [1] is applicable and shows that the obvious isomorphism $] Z\left[_{\hat{F}_{1}} \rightarrow\right] Z\left[_{\hat{F}_{2}}\right.$ extends to an isomorphism of strict neighborhoods. In particular the dagger spaces are the same.

This being said we continue the proof. We fix an index $i_{0} \in J$. If $J=\left\{i_{0}\right\}$ the assertion follows from the proof of [2] Prop. 1.10. By the choice of our covering $A_{J}$ is the localization of $A_{i_{0}}$ by an element $g \in A_{i_{0}}$. We take a lift $\tilde{g} \in B_{i_{0}}$ and we set $B_{i_{0}}^{\prime}=\left(B_{i_{0}}\right) \tilde{g}$. Then $F_{i_{0}}^{\prime}=\operatorname{Spec} B_{i_{0}}^{\prime}$ is a standard smooth scheme over $W$ which lifts $X_{J}$.

We set $E=\prod_{i \in J, i \neq i_{0}} F_{i}$. By the strong fibration theorem above the special frames $\left(X_{J}, F_{i_{0}} \times E\right)$ and $\left(X_{J}, F_{i_{0}}^{\prime} \times E\right)$ have isomorphic dagger spaces. It is enough to consider the latter one. Since $E$ is standard smooth we can choose an étale morphism $E \rightarrow \mathbb{A}_{W}^{n}$ for some number $n$. Again by the strong fibration theorem it is enough to prove our proposition for the special frame $\left(X_{J}, F_{i_{0}}^{\prime} \times \mathbb{A}_{W}^{n}\right)$.

We may assume the map $X_{J} \rightarrow \mathbb{A}_{W}^{n}$ induced by the last special frame factors over the zero section Spec $k \rightarrow \mathbb{A}_{W}^{n}$. This is seen by a simple coordinate change. Consider the comorphism of the closed embedding $X_{J} \rightarrow F_{i_{0}}^{\prime} \times \mathbb{A}_{W}^{n}$ :

$$
B_{i_{0}}^{\prime}\left[X_{1}, \ldots, X_{n}\right] \stackrel{\gamma}{\rightarrow} A_{J} .
$$

We find elements $b_{i} \in B_{i_{0}}^{\prime}$ such that $\gamma\left(b_{i}\right)=\gamma\left(X_{i}\right)$. Since we may take $X_{i}^{\prime}=X_{i}-b_{i}$, $i=1, \ldots, n$ as new indeterminates on the left hand side of (4.36) we see that our original 
special frame is isomorphic to one of the required form. Our proof will be finished by the Corollary 4.38 of the following:

Proposition 4.37. - Let $\breve{D}=\{z \in \bar{K}|| z \mid<1\}$ be the open unit ball with its natural dagger space structure. Let $n$ be a natural number. Let $Q=\operatorname{Sp}^{\dagger} A$ be a smooth affinoid dagger space, such that $\Omega_{Q}^{1}$ is a free $\theta$-module. Then the following hold:

1. $H^{0}\left(Q, \Omega_{Q}\right) \rightarrow H^{0}\left(Q \times \breve{D}^{n}, \Omega_{Q \times \breve{D}^{n}}\right)$ is a quasiisomorphism of complexes.

2. The complex $H^{1}\left(Q \times \breve{D}^{n}, \Omega_{Q \times \breve{D}^{n}}\right)$ is acyclic.

3. $H^{i}\left(Q \times \breve{D}^{n}, \Omega_{Q \times \breve{D}^{n}}^{q}\right)=0$ for $i \geq 2$ and all $q$.

This proposition is inspired by [2] Thm. 1.4. We postpone its proof to the end.

Corollary 4.38. - Let $Z=\operatorname{Spec} A$ be a smooth affine scheme over $k$. Let $F=\operatorname{Spec} B$ be a smooth affine scheme which lifts $A$. Let $Q=] Z\left[_{\hat{F}}^{\dagger}\right.$ be the tubular neighborhood with its dagger space structure. We consider the constant map to the origin $Z \rightarrow \mathbb{A}_{W}^{n}$.

The dagger space associated to the special frame $\left(Z, F \times \mathbb{A}_{W}^{n}\right)$ is $Q \times \breve{D}^{n}$. Let

$$
Q \times \breve{D}^{n} \rightarrow Z
$$

be the specialization map.

Then the natural morphism

$$
\mathbf{s p}_{*} \Omega_{Q \times \breve{D}^{n}} \rightarrow \mathbf{R} \mathbf{s p}_{*} \Omega_{Q \times \breve{D}^{n}}
$$

is a quasiisomorphism.

Proof. - We consider the spectral sequence of hypercohomology

$$
\mathcal{H}^{q}\left(R^{p} \mathbf{s p}_{*} \Omega_{Q \times \breve{D}^{n}}\right) \Rightarrow \mathbf{R}^{p+q} \mathbf{s p}_{*} \Omega_{Q \times \breve{D}^{n}} .
$$

For an affine subset $U \subset Z$ the inverse image $U \subset Q$ by sp : $Q \rightarrow Z$ is an affinoid dagger space. Choose $U$ sufficiently small, such that $\Omega_{\mathcal{U}}$ is free.

By Proposition 4.37 the complex $H^{p}\left(U \times \breve{D}^{n}, \Omega_{U \times \breve{D}^{n}}\right)$ is acyclic for $p \geq 1$. It follows that the complexes $R^{p} \mathbf{s p}_{*} \Omega_{Q \times \breve{D}^{n}}$ are acyclic. Therefore the spectral sequence (4.39) degenerates. This proves the corollary and Proposition 4.35.

THEOREM 4.40. - Let $X$ be a smooth quasiprojective scheme over $k$. Then we have a natural quasiisomorphism

$$
R \Gamma_{\text {rig }}(X) \rightarrow \mathbf{R} \Gamma\left(X, W^{\dagger} \Omega_{X / k}\right) \otimes \mathbb{Q} .
$$

Proof. - We choose a covering $\left\{X_{i}\right\}_{i \in I}$ as above. We consider the simplicial scheme $\mathfrak{X}_{\bullet}=\left\{X_{J}\right\}_{J \subset I}$ and its natural augmentation $\epsilon: \mathfrak{X}_{\bullet} \rightarrow X$. We set $F_{J}=\prod_{i \in J} F_{i}$. Then we obtain a simplicial object of frames $\left(X_{J}, F_{J}\right)$ which gives rise to a simplicial dagger space $\mathfrak{Q}_{\bullet}=\left\{Q_{J}\right\}$. For each $J \subset I$ we have the comparison morphism (4.32)

$$
\mathbf{s p}_{*} \Omega_{Q_{J}} \rightarrow W^{\dagger} \Omega_{X_{J} / k} \otimes \mathbb{Q} \text {. }
$$

This glues to a morphism of simplicial sheaves

$$
\mathbf{s p}_{*} \Omega_{\mathfrak{Q}} \rightarrow W^{\dagger} \Omega_{\mathfrak{X} \bullet / k} \otimes \mathbb{Q} \text {. }
$$

$4^{\text {e }}$ SÉRIE - TOME $44-2011-$ No $^{\circ} 2$ 
By Proposition 4.35 and Proposition 4.31 this gives a quasiisomorphism

$$
\begin{gathered}
\mathbf{R} \mathbf{s p}_{*} \Omega_{\mathfrak{Q}_{\bullet}} \rightarrow W^{\dagger} \Omega_{\mathfrak{X}_{\bullet} / k} \otimes \mathbb{Q} \\
\mathbf{R} \epsilon_{*} \mathbf{R} \mathbf{s p} \Omega_{\mathfrak{Q}_{\bullet}} \cong \mathbf{R} \epsilon_{*} W^{\dagger} \Omega_{X \cdot / k} \otimes \mathbb{Q} \cong W^{\dagger} \Omega_{X / k} \otimes \mathbb{Q} .
\end{gathered}
$$

We will verify that the left hand side of (4.41) is a complex on $X$ whose hypercohomology is rigid cohomology. We consider a frame $\mathbf{P}: X \rightarrow \bar{X} \rightarrow \hat{P}$ which gives the rigid cohomology of $X$. If $\mathbf{P}^{\prime}: X \rightarrow \bar{X}^{\prime} \rightarrow \hat{P}^{\prime}$ is a second frame we may form the product as follows: We consider the closure $\bar{X}^{\prime \prime}$ of $X$ in $\bar{X}^{\prime} \times \bar{X}^{\prime \prime}$. Then we obtain a new frame $X \rightarrow \bar{X}^{\prime \prime} \rightarrow \hat{P} \times \hat{P}^{\prime}$. We denote this frame by $\mathbf{P} \times \mathbf{P}^{\prime}$.

By [4] we find a simplicial frame $\left\{\mathbf{P}_{J}\right\}$ where $\mathbf{P}_{J}$ is a frame for $X_{J}$ with an augmentation to $\mathbf{P}$. To the frames $\left(X_{J}, F_{J}\right)$ we may associate functorially frames $\mathbf{Q}_{J}$. We obtain a commutative diagram of simplicial schemes

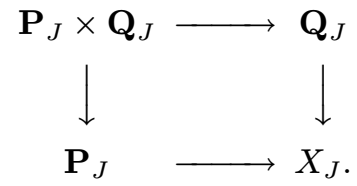

Consider the corresponding diagram of dagger spaces. Since each of these dagger space gives the rigid cohomology of $X_{J}$ we obtain quasiisomorphisms

$$
R \mathbf{s p}_{*} \Omega_{\mathbf{Q}_{J}} \longleftarrow R \mathrm{sp}_{*} \Omega_{\mathbf{R}_{J}} \longrightarrow R \mathrm{sp}_{*} \Omega_{\mathbf{P}_{J}} .
$$

Here $\mathbf{R}_{J}$ denotes the dagger space associated with $\mathbf{P}_{J} \times \mathbf{Q}_{J}$. But this implies that we obtain quasiisomorphisms of simplicial sheaves too:

$$
\mathbf{R} \mathbf{s p}_{*} \Omega_{\mathbf{Q}_{\bullet}} \longleftarrow \mathbf{R} \mathbf{s p}_{*} \Omega_{\mathbf{R}_{\bullet}} \longrightarrow \mathbf{R} \mathbf{s p}_{*} \Omega_{\mathbf{P}_{\bullet}} .
$$

If we apply $\mathbf{R} \Gamma\left(R \epsilon_{*}\right.$, ?) to the last complex in (4.42) we obtain a quasiisomorphism with $R \Gamma_{\text {rig }}(X)$ by [4]. Together with (4.41) this proves the theorem.

It remains to prove Proposition 4.37. Let $Q=\operatorname{Sp}^{\dagger} A$ be a reduced affinoid dagger space. Recall that $A$ is a weakly complete finitely generated algebra tensored with $\mathbb{Q}[14]$. We represent $A$ as a quotient

$$
\kappa: K\left\langle X_{1}, \ldots X_{m}\right\rangle^{\dagger} \rightarrow A .
$$

The algebra on the left hand side is the union of the algebras for real numbers $\epsilon>0$

$$
K\left\langle X_{1}, \ldots X_{m}\right\rangle_{\epsilon}=\left\{\sum_{I} c_{I} X^{I} \subset K\left[\left[X_{1}, \ldots X_{m}\right]\right]\left|\operatorname{ord}_{p} c_{I}-\epsilon\right| I \mid \rightarrow \infty\right\} .
$$

This is a Tate algebra if $\epsilon \in \mathbb{Q}[3]$ 6.1.5. We denote by $\tilde{\gamma}_{\epsilon}$ the Gauss norm on this ring:

$$
\tilde{\gamma}_{\epsilon}\left(\sum_{I} c_{I} X^{I}\right)=\inf _{I}\left\{\operatorname{ord}_{p} c_{I}-\epsilon|I|\right\} \text {. }
$$

Let $A_{\epsilon}$ be the image of $K\left\langle X_{1}, \ldots X_{m}\right\rangle_{\epsilon}$ by $\kappa$. We denote by $\gamma_{\epsilon}$ the quotient norm on $A_{\epsilon}$. Since $A_{\epsilon}$ is reduced by assumption $\gamma_{\epsilon}$ is equivalent to the spectral norm $\sigma_{\epsilon}$ on $A_{\epsilon}$.

Let $D=\operatorname{Sp}^{\dagger} K\langle X\rangle^{\dagger}$ be the closed dagger disc. We write

$$
A\left\langle T_{1}, \ldots, T_{n}\right\rangle^{\dagger}:=\Gamma\left(Q \times D^{n}, \theta\right) .
$$


It follows from the definitions that $A\left\langle T_{1}, \ldots, T_{n}\right\rangle^{\dagger}$ consists of all power series $\sum_{J} a_{J} T^{J} \in(A \otimes \mathbb{Q})\left[\left[T_{1}, \ldots, T_{n}\right]\right]$ such that there are an $\epsilon>0$ and a number $C$ with $a_{J} \in A_{\epsilon}$ for all $J \in \mathbb{Z}_{\geq 0}^{n}$ and such that

$$
\sigma_{\epsilon}\left(a_{J}\right)-\epsilon|J| \geq C
$$

In this condition we could replace $\sigma_{\epsilon}$ by $\gamma_{\epsilon}$.

Lemma 4.44. - Let $Q=\operatorname{Sp}^{\dagger} A$ and let $\breve{D}$ be the open dagger disc. Then the algebra $\Gamma\left(Q \times \breve{D}^{n}\right)$ consists of all power series

$$
\sum_{J} a_{J} T^{J} \in(A \otimes \mathbb{Q})\left[\left[T_{1}, \ldots, T_{n}\right]\right]
$$

such that for each $\delta>0$ there are an $\epsilon>0$ and a constant $C$ such that for all $J$ we have that $a_{J} \in A_{\epsilon}$ and that

$$
\sigma_{\epsilon}\left(a_{J}\right)+\delta|J| \geq C
$$

Proof. - Indeed, let $D_{\delta}=\left\{z \in \bar{K} \mid \operatorname{ord}_{p} z \geq \delta\right\}$ be the closed dagger disc. Then $\Gamma\left(Q \times D_{\delta}^{n}, \theta\right)$ consists of all power series $\sum_{J} a_{J} T^{J}$ such that there are an $\epsilon>0$ and a constant $C$ with

$$
\sigma_{\epsilon}\left(a_{J}\right)+\delta|J|-\epsilon|J| \geq C
$$

This implies the result.

Lemma 4.45. - Let $Q=\mathrm{Sp}^{\dagger} A$ and let $D$ be the closed dagger disc. Let

$$
\Lambda_{n}=\bigoplus_{i_{1}<\cdots<i_{k}} \Gamma\left(Q \times D^{n}\right) d T_{i_{1}} \wedge \cdots \wedge d T_{i_{k}}
$$

be the complex with the obvious differential.

Then the complex $A \rightarrow \Lambda_{n}$ is acyclic.

Proof. - We consider $\Lambda_{n}$ as a multicomplex with the partial differentials $\partial_{i}, i=1, \ldots, n$. Let $\widetilde{\Lambda}_{n} \subset \Lambda_{n}$ be the direct summands of 4.46 with $i_{k}<n$.

It suffices to show that the following complex is exact:

$$
0 \rightarrow \Lambda_{n-1} \rightarrow \widetilde{\Lambda}_{n} \stackrel{\partial_{n}}{\rightarrow} \widetilde{\Lambda}_{n} \rightarrow 0 .
$$

The only nontrivial thing to show is that an expression $f d X_{n}$, with $f \in \Gamma\left(Q \times D^{n}\right)$, is the partial differential of some $g \in \Gamma\left(Q \times D^{n}\right)$. We set $f=\sum_{J} a_{J} T^{J}$. We denote by $e$ the vector $(0, \ldots, 0,1) \in \mathbb{Z}^{n}$, and we denote by $j_{n}$ the last entry of the vector $J$. We have to show that the power series

$$
\sum_{J} \frac{a_{J}}{j_{n}+1} T^{J+e}
$$

is in $\Gamma\left(Q \times D^{n}\right)$.

By (4.43) we find $\epsilon>0$ and $C$ such that

$$
\sigma_{\epsilon}\left(a_{J}\right)-\epsilon|J| \geq C .
$$

$4^{\mathrm{e}}$ SÉRIE - TOME $44-2011$ - No 2 
We take $0<\epsilon^{\prime}<\epsilon$. We note that $A_{\epsilon} \subset A_{\epsilon^{\prime}}$ and that $\sigma_{\epsilon^{\prime}} \geq \sigma_{\epsilon}$. Since $\sigma_{\epsilon}$ is multiplicative we find

$$
\begin{aligned}
\sigma_{\epsilon^{\prime}}\left(\frac{a_{J}}{j_{n}+1}\right)-\epsilon^{\prime}|J+e| & =\sigma_{\epsilon^{\prime}}\left(a_{J}\right)-\operatorname{ord}_{p}\left(j_{n}+1\right)-\epsilon^{\prime}(|J|+1) \\
& \geq \sigma_{\epsilon}\left(a_{J}\right)-\epsilon(|J|+1)+\left(\epsilon-\epsilon^{\prime}\right)(|J|+1)-\operatorname{ord}_{p}\left(j_{n}+1\right) \\
& \geq C-\epsilon+\left(\epsilon-\epsilon^{\prime}\right)\left(j_{n}+1\right)-\operatorname{ord}_{p}\left(j_{n}+1\right) .
\end{aligned}
$$

It is clear that the last expression is bounded below independent of $J$.

We have the same for the open disc $\breve{D}$.

Lemma 4.47. - With the same notations as before let

$$
\breve{\Lambda}_{n}=\bigoplus_{i_{1}<\cdots<i_{k}} \Gamma\left(Q \times \breve{D}^{n}\right) d T_{i_{1}} \wedge \cdots \wedge d T_{i_{k}}
$$

be the complex with the obvious differential.

Then the complex $A \rightarrow \breve{\Lambda}_{n}$ is acyclic.

Proof. - As in the proof of the last lemma the only nontrivial thing to show is that an expression $f d X_{n}$, with $f \in \Gamma\left(Q \times \breve{D}^{n}\right)$, may be written $f d X_{n}=\partial_{n} g$ for some $g \in \Gamma\left(Q \times \breve{D}^{n}\right)$. We have to show that the power series

$$
\sum_{J} \frac{a_{J}}{j_{n}+1} T^{J+e}
$$

is in $\Gamma\left(Q \times \breve{D}^{n}\right)$. We apply Lemma 4.44. Assume $\delta>0$ is given. We take any $\delta^{\prime}<\delta$. Then we find $\epsilon>0$ and a constant $C$ such that

$$
\sigma_{\epsilon}\left(a_{J}\right)+\delta^{\prime}|J| \geq C
$$

We see that the following expression is bounded below:

$$
\sigma_{\epsilon}\left(\frac{a_{J}}{j_{n}+1}\right)+\delta(|J+e|)=\sigma_{\epsilon}\left(a_{J}\right)-\operatorname{ord}_{p}\left(j_{n}+1\right)+\left(\delta-\delta^{\prime}\right)(|J|+1) \delta^{\prime}(|J|+1) .
$$

We come now to the proof of Proposition 4.37. We write

$$
\breve{D}^{n}=\bigcup_{i=1}^{\infty} U_{i}
$$

as a union of dagger balls of ascending radius. For an abelian sheaf $\mathscr{F}$ on $\breve{D}$ we define the sheaves $\mathscr{C}^{0}(\mathcal{F})=\mathscr{C}^{1}(\mathcal{F})$ :

$$
\mathscr{C}^{i}(\mathcal{F})(V)=\prod_{t=1}^{\infty} \mathcal{F}\left(U_{t} \cap V\right) .
$$

We obtain a resolution of $\mathcal{F}$

$$
\begin{aligned}
0 \rightarrow \mathcal{F} \rightarrow \mathscr{C}^{0}(\mathcal{F}) & \rightarrow \mathscr{C}^{1}(\mathcal{F}) \rightarrow 0 \\
\prod s_{t} & \mapsto \prod\left(s_{t}-s_{t+1}\right)
\end{aligned}
$$


If $\mathcal{F}$ is a coherent $\Theta_{Q \times \breve{D}^{n}}$-module the cohomology groups $H^{p}\left(Q \times U_{t}, \mathcal{F}\right)$ vanish for $p \geq 1$ by Tate-acyclicity for affinoid dagger spaces [6]. Therefore $\mathbf{R} \Gamma\left(Q \times \breve{D}^{n}, \mathcal{F}\right)$ is represented by the global sections of the complex (4.49)

$$
\begin{aligned}
\prod_{t=1}^{\infty} \mathcal{F}\left(Q \times U_{t}\right) & \rightarrow \prod_{t=1}^{\infty} \mathcal{F}\left(Q \times U_{t}\right) \\
\prod s_{t} & \mapsto \prod\left(s_{t}-s_{t+1}\right) .
\end{aligned}
$$

This proves already the third assertion of Proposition 4.37.

Let $\pi: Q \times U_{t} \rightarrow Q$ be the projection. We write

$$
G_{t}^{p}=\left(\pi^{*} \Omega_{Q}^{p}\right)\left(Q \times U_{t}\right) .
$$

This is a free module over $H^{0}\left(Q \times U_{t}, \theta\right)$ by assumption. With this notation the complex $H^{0}\left(Q \times U_{t}, \Omega_{Q \times U_{t}}\right)$ is represented by the double complex with the components

$$
C^{p, q}\left(U_{t}\right)=\oplus_{i_{1}<\ldots i_{q}} G_{t}^{p} d T_{i_{1}} \wedge \cdots \wedge d T_{i_{q}} .
$$

The map (4.50) induces a morphism of complexes

$$
\prod_{t=1}^{\infty} C^{p, q}\left(U_{t}\right) \rightarrow \prod_{t=1}^{\infty} C^{p, q}\left(U_{t}\right) .
$$

The kernel resp. the cokernel of the induced map of total complexes is the complex $H^{0}\left(Q \times \breve{D}^{n}, \Omega_{Q \times \breve{D}^{n}}\right)$ resp. $H^{1}\left(Q \times \breve{D}^{n}, \Omega_{Q \times \breve{D}^{n}}\right)$.

By Lemma 4.45 the complex $C^{p, \cdot}\left(U_{t}\right)$ for fixed $p$ is quasiisomorphic to $H^{0}\left(Q, \Omega_{Q}^{p}\right)$ regarded as a complex concentrated in degree zero. Therefore the total complex of $C^{p, q}\left(U_{t}\right)$ is quasiisomorphic to the complex $H^{0}\left(Q, \Omega_{Q}\right)$.

We consider the projection $\pi: Q \times \breve{D}^{n} \rightarrow Q$ and write

$$
\tilde{G}^{p}=\left(\pi^{*} \Omega_{Q}^{p}\right)\left(Q \times \breve{D}^{n}\right) .
$$

By assumption these are free modules over $H^{0}\left(Q \times \breve{D}^{n}, \theta\right)$.

Then we may represent $H^{0}\left(Q \times \breve{D}^{n}, \Omega_{Q \times \breve{D}^{n}}\right)$ by the double complex with components

$$
B^{p, q}=\oplus_{i_{1}<\ldots i_{q}} \tilde{G}^{p} T_{i_{1}} \wedge \cdots \wedge d T_{i_{q}} .
$$

Lemma 4.47 asserts that the total complex of $B^{p, q}$ is quasiisomorphic to the complex $H^{0}\left(Q, \Omega_{Q}\right)$. This proves the first assertion of Proposition 4.37 .

We deduce finally that the complex $H^{1}\left(Q \times \breve{D}^{n}, \Omega_{Q \times \breve{D}^{n}}\right)$ is quasiisomorphic to the total complex of the triple complex

$$
B^{p, q} \rightarrow \prod_{t=1}^{\infty} C^{p, q}\left(U_{t}\right) \rightarrow \prod_{t=1}^{\infty} C^{p, q}\left(U_{t}\right) .
$$

By what we already proved the last complex is quasiisomorphic to the total complex of the double complex

$$
\begin{aligned}
H^{0}\left(\mathcal{Q}, \Omega_{Q}\right) \rightarrow \prod_{t=1}^{\infty} H^{0}\left(\mathcal{Q}, \Omega_{Q}\right) & \rightarrow \prod_{t=1}^{\infty} H^{0}\left(\mathcal{Q}, \Omega_{Q}\right), \\
\prod s_{t} & \mapsto \prod^{\left(s_{t}-s_{t+1}\right)}
\end{aligned}
$$


where the first embedding is diagonal. But the total complex is acyclic because the double complex is already acyclic with respect to the horizontal differential. This proves the second assertion and finishes the proof of Proposition 4.37.

Acknowledgments. - We are very grateful to the referee who made many substantial remarks that guided us to finish this paper. We thank Ahmed Abbes, Elmar Grosse-Klönne, Lars Hesselholt, and Nobuo Tsuzuki for very helpful discussions. The first author's work on the subject began as part of his Ph.D. thesis. He is grateful to his thesis advisor, Kiran Kedlaya, for introducing him to the topic and for his many hours of assistance.

\section{REFERENCES}

[1] P. Berthelot, Cohomologie rigide et cohomologie rigide à supports propres, preprint 96-03 de l'université de Rennes, http://perso.univ-rennes1.fr/pierre. berthelot/publis/Cohomologie_Rigide_I.pdf, 1996.

[2] P. Berthelot, Finitude et pureté cohomologique en cohomologie rigide, Invent. Math. 128 (1997), 329-377.

[3] S. Bosch, U. Güntzer, R. Remmert, Non-Archimedean analysis, Grund. Math. Wiss. 261, Springer, 1984.

[4] B. Chiarellotto, N. Tsuzuki, Cohomological descent of rigid cohomology for étale coverings, Rend. Sem. Mat. Univ. Padova 109 (2003), 63-215.

[5] C. Davis, A. Langer, T. Zink, Overconvergent Witt vectors, preprint http://www . math.uci.edu/ davis/DLZOCW.pdf.

[6] E. Grosse-KLönne, Rigid analytic spaces with overconvergent structure sheaf, J. reine angew. Math. 519 (2000), 73-95.

[7] A. Grothendieck, J. Dieudonné, Éléments de géométrie algébrique, Publ. Math. I.H.É.S. 4, 8, 11, 17, 20, 24, 28, 32 (1960-67).

[8] L. Illusie, Complexe de de Rham-Witt et cohomologie cristalline, Ann. Sci. École Norm. Sup. 12 (1979), 501-661.

[9] K. S. Kedlaya, More étale covers of affine spaces in positive characteristic, J. Algebraic Geom. 14 (2005), 187-192.

[10] A. Langer, T. ZINK, De Rham-Witt cohomology for a proper and smooth morphism, J. Inst. Math. Jussieu 3 (2004), 231-314.

[11] A. Langer, T. Zink, Gauss-Manin connection via Witt-differentials, Nagoya Math. J. 179 (2005), 1-16. 
[12] S. LubKin, Generalization of $p$-adic cohomology: bounded Witt vectors. A canonical lifting of a variety in characteristic $p \neq 0$ back to characteristic zero, Compositio Math. 34 (1977), 225-277.

[13] D. Meredith, Weak formal schemes, Nagoya Math. J. 45 (1972), 1-38.

[14] P. Monsky, G. Washnitzer, Formal cohomology. I, Ann. of Math. 88 (1968), 181217.

(Manuscrit reçu le 22 juillet 2008; accepté, après révision, le 12 juillet 2010.)

\author{
Christopher Davis \\ Massachusetts Institute of Technology \\ Department of Mathematics \\ Cambridge, MA 02139, USA \\ E-mail: davis@math.mit.edu \\ Andreas LANGER \\ University of Exeter \\ Mathematics \\ Exeter EX4 4QF, Devon, UK \\ E-mail: a. langer@exeter.ac.uk \\ Thomas ZINK \\ Fakultät für Mathematik \\ Universität Bielefeld \\ Postfach 100131 \\ D-33501 Bielefeld, Germany \\ E-mail: zink@math.uni-bielefeld.de
}

